\title{
GRAVITATIONAL WAVES FROM AXISYMMETRIC, ROTATING STELLAR CORE COLLAPSE
}

\author{
Christian D. OTT \\ Institut für Theoretische Astrophysik, Universität Heidelberg, \\ Heidelberg, Germany; cott@ita.uni-heidelberg.de \\ ADAM BURROWS \\ Steward Observatory, The University of Arizona, Tucson, AZ 85721; burrows @ zenith.as.arizona.edu \\ ELI LIVNE \\ Hebrew University, Jerusalem, Israel; eli@ frodo.fiz.huji.ac.il \\ ROLF WALDER \\ Steward Observatory, The University of Arizona, Tucson, AZ 85721; rwalder@as.arizona.edu
}

Submitted to APJ: July 26, 2003

ACCEPTED: SEPTEMBER 14, 2003

\begin{abstract}
We have carried out an extensive set of two-dimensional, axisymmetric, purely-hydrodynamic calculations of rotational stellar core collapse with a realistic, finite-temperature nuclear equation of state and realistic massive star progenitor models. For each of the total number of 72 different simulations we performed, the gravitational wave signature was extracted via the quadrupole formula in the slow-motion, weak-field approximation. We investigate the consequences of variation in the initial ratio of rotational kinetic energy to gravitational potential energy and in the initial degree of differential rotation. Furthermore, we include in our model suite progenitors from recent evolutionary calculations that take into account the effects of rotation and magnetic torques. For each model, we calculate gravitational radiation wave forms, characteristic wave strain spectra, energy spectra, final rotational profiles, and total radiated energy. In addition, we compare our model signals with the anticipated sensitivities of the 1st- and 2nd-generation LIGO detectors coming on line. We find that most of our models are detectable by LIGO from anywhere in the Milky Way.
\end{abstract}

Subject headings: supernovae: gravitational radiation, stars: rotation

\section{INTRODUCTION}

The classical constraints on core-collapse supernova theory are nucleosynthetic yields, residue neutron star or black hole mass, explosion energy, neutrino signal, pulsar fields, and pulsar kicks. Any viable theory of supernova explosions must in the long run reproduce these data. However, we are still a long way from this situation and much work remains before the roles of neutrinos, multi-dimensional hydrodynamics, rotation, convection, and magnetic fields in the mechanism of core-collapse supernovae are fully elucidated (Bethe and Wilson 1985; Herant et al. 1994; Burrows, Hayes, and Fryxell 1995; Fryer et al. 1999; Fryer and Heger 2000; Rampp and Janka 2000,2002; Liebendörfer et al. 2001a,b; Buras et al. 2003; Thompson, Burrows, and Pinto 2003; Akiyama et al. 2003).

There is, however, another quite dramatic potential constraint on core-collapse supernovae: their gravitational radiation signatures. Massive stars (ZAMS mass $\gtrsim 8 \mathrm{M}_{\odot}$ ) develop degenerate cores in the final stages of nuclear burning and achieve the Chandrasekhar mass. Gravitational collapse ensues, leading to dynamical compression to nuclear densities, subsequent core bounce, and hydrodynamical shock wave generation. These phenomena involve large masses at high velocities $(\sim c / 4)$ and great accelerations. Such dynamics, if only slightly aspherical, will lead to copious gravitational wave emission and, arguably, to one of the most distinctive features of core-collapse supernovae. The gravitational waveforms and associated spectra bear the direct stamp of the hydrodynamics and rotation of the core and speak volumes about internal supernova evolution. Furthermore, they provide data that complement (temporally and spectrally) those from the neutrino pulse (which also originates from the core), enhancing the diagnostic potential of each.

As the current generation of gravitational wave detectors comes on line, gravitational wave astronomy might soon be able to shed light on the supernova phenomenon. Gravitational waves couple so weakly to matter that they propagate almost undistorted from their source in the ultra-dense collapsing and rebounding stellar core to detectors like LIGO (Gustafson et al. 1999), VIRGO (Punturo 2003), GEO600 (Willke et al. 2002), and TAMA (Ando et al. 2001) on Earth. No other physical signal, apart from neutrinos, can give comparable "live" dynamical data of a star's death. However, there is a major caveat: in order for gravitational waves to be emitted the collapsing core must have a sizable and rapidly varying asphericity, since grav- 
Wheeler 1973).

Fortunately, as astronomical observations have shown, most stars rotate (Fukuda 1982; Heger et al. 2003). Rotation can result in large asphericity at and around bounce and, hence, provides hope that the emission of gravitational radiation from stellar core collapse can be significant. Furthermore, RayleighTaylor-like convection in the protoneutron star, the aspherical emission of neutrinos and post-bounce triaxial rotational instabilities are also potential sources of gravitational radiation. Together these phenomena, with their characteristic spectral and temporal signatures, make core-collapse supernovae promising and interesting generators of gravitational radiation.

Early studies of gravitational wave emission from stellar core collapse used either spherically symmetric models and applied perturbation techniques to analyze the gravitational radiation (Turner and Wagoner 1979; Seidel and Moore 1987; Seidel, Myra, and Moore 1987) or applied semi-analytic methods to the aspherical Newtonian collapse of homogeneous and inhomogeneous, uniformly-rotating, and degenerate ellipsoids (Shapiro 1977; Saenz and Shapiro 1978,1979,1981; Moncrief 1979; Ipser and Managan 1984). Subsequent studies were based on 2-D Newtonian hydrodynamic core-collapse calculations with rotation and various simple treatments of the microphysics. Müller and Hillebrandt (1981) and Müller (1982) performed a limited set of simulations with a finite-temperature equation of state (EOS) using initial models from stellar evolution calculations, and did not treat the neutrino physics. Finn and Evans (1990), Bonazzola and Marck (1993), Yamada and Sato (1995) and Zwerger and Müller (1997) studied the gravitational wave signature from collapsing $n=3$ polytropes with a simplified equation of state, consisting of a polytropic and a thermal part. In all of the above studies, rotation was imposed upon spherically symmetric initial models by an artificial rotation law. Zwerger and Müller (1997) performed the most comprehensive sweep through values of the rotation parameter $\beta$, defined by

$$
\beta=\frac{E_{\text {rot }}}{\left|E_{\text {grav }}\right|},
$$

where $E_{r o t}$ is the total rotational kinetic energy and $E_{\text {grav }}$ is the total gravitational energy. They also varied the degree of initial differential rotation and used self-consistent initial models in rotational equilibrium, calculated using the method of Eriguchi and Müller (1985). Dimmelmeier et al. (2001a,b) extended the aforementioned study to general-relativistic gravity in the conformally-flat limit.

Mönchmeyer et al. (1991) accounted for electron capture on protons and employed an approximate neutrino leakage scheme. Their limited set of detailed 2-D calculations used rotationally non-equilibrium initial models from stellar evolution calculations and a finite-temperature EOS (Hillebrandt and Wolff 1985). Using a smooth particle hydrodynamics code with a neutrino diffusion scheme and the Lattimer-Swesty EOS (Lattimer and Swesty 1991), Fryer and Heger (2000) performed 2-D rotating collapse simulations. Fryer, Holz, and Hughes (2002) followed this up by computing the corresponding gravitational wave signature.

In a recent Newtonian study, Kotake, Yamada, and Sato (2003) used an EOS based on the relativistic mean field theory (Shen et al. 1998). They took electron capture into account and made use of a leakage scheme for simplified neutrino transport.
They performed a limited set of calculations that employed realistic $15 \mathrm{M}_{\odot}$ ZAMS progenitor models of Woosley and Weaver (1995) onto which they imposed rotation by rotation laws similar to those of Mönchmeyer et al. (1991).

While most of the above studies were primarily concerned with the gravitational wave signatures due to core bounce itself, others have highlighted the gravitational wave signatures from later stages of supernova evolution and due to other phenomena. Epstein (1978), Burrows and Hayes (1996), and Müller and Janka (1997) explored the gravitational wave signature of anisotropic neutrino emission. Burrows and Hayes (1996) and Müller and Janka (1997) studied aspherical convective motion behind the shock and in the protoneutron star. Rampp, Müller, and Ruffert (1998), Brown (2001), Centrella et al. (2001), and Shibata, Karino and Eriguchi $(2002,2003)$ investigated triaxial instabilities in the quickly spinning neutron star remnant (New 2003).

In this paper, we present the results from our 2-D axisymmetric purely hydrodynamical simulations of rotating stellar core collapse, performed with the code VULCAN/2D (Livne 1993). For this study, we used the 11,15 and $20 \mathrm{M}_{\odot}$ progenitor models of Woosley and Weaver (1995). We imposed rotation using the same prescriptions employed by Zwerger and Müller (1997) and Dimmelmeier, Font, and Müller (2002b). In addition, we used the recent rotating progenitor models of Heger, Langer, and Woosley (2000) and Heger et al. (2003). The latter are 1D calculations that employ a prescription for angular momentum transport and mass loss in the evolving massive star, but do not include the back-reaction of centrifugal effects on the dynamics after carbon burning. All of our calculations, except for the comparison studies to previous work, have made use of the Lattimer-Swesty EOS in tabular form (Thompson, Burrows, and Pinto 2003), as well as realistic progenitor structures. We have not included any approximate treatment of electron capture and neutrino transport since these schemes (e.g., the leakage scheme used in Kotake, Yamada, and Sato 2003) only crudely approximate full neutrino transport.

In $\S 2$, we review the progenitor model suite we have relied upon. Section 3 summarizes the initial model rotational law that we used to set models into rotation. In $\S 4$, we provide an overview of our implementation of VULCAN/2D and discuss the two different equations of state, realistic and polytropic, that we have used. Section 4.3 deals with our method for the extraction of the gravitational wave signature from the hydrodynamic data. Section 5 covers our calculations with polytropic models and polytropic equations of state. These are provided and compared with previous work in order to validate the methods and codes we have employed. In $\S 6$, our results with more realistic progenitors and a more detailed equation of state are presented and compared with those of previous studies. We discuss the hydrodynamics of rotating collapse, the generation of vortices, the damping effect of a stalled shock wave, and the excitation of $l=2$ pulsations, as well as the signatures of these hydrodynamic features in the gravitational wave pulse. Importantly, we provide model gravitational wave spectra and estimate the detectability of these signals in the LIGO detector (Gustafson et al. 1999). We verify the two major types of wave forms and post-bounce behaviors originally identified by $\mathrm{Zw}$ erger and Müller (1997) that depend upon the initial rotational energy and the degree of differential rotation. Moreover, we derive the progenitor model dependence of the gravitiational wave 
signatures. In §7, we discuss the angular velocity profiles associated with a subset of the models we have studied, identifying in particular the maximum spin rate and the magnitude and position of the maximum rotational shear that result from collapse. Section 8 provides both a context in which to discuss the detection of the gravitational wave signatures of collapse and estimates of their detectability in the galaxy. In $\S 9$, we summarize our conclusions concerning the generic features of the gravitational wave signature of core-collapse supernovae. A major goal of our study is the illumination of the importance of using realistic progenitors and equations of state when deriving and analyzing the gravitational wave forms and spectra from supernova explosions. Signal templates derived using polytropes and a polytropic EOS can deviate significantly from those obtained using more realistic assumptions and starting points. A further goal of our study is the derivation of the systematic variation with the degree of initial rotation of a supernova's gravitational wave form and its spectrum.

\section{PROGENITOR MODELS}

Since this study is concerned with the gravitational wave signal originating from the highly dynamical aspherical bulk mass motions at core collapse and bounce and does not aim at the solution of the full supernova problem, it is sensible to restrict our simulations to the modeling of the central rotating iron core whose hydrodynamical collapse and bounce is believed to yield the dominant contribution to the gravitational wave energy emitted during the supernova phenomenon. A simple approach for constructing progenitor data used in many previous studies is to approximate the iron core as an ultra-relativistic, electron-degenerate Chandrasekhar core with a central density of $10^{10} \mathrm{~g} \mathrm{~cm}^{-3}$ and an electron number fraction $\left(Y_{e}\right)$ of 0.5 . The equation of state for such a core is then polytropic with a $\Gamma$ of $4 / 3(n=3)$ and a polytropic constant $K$ given by eq. (10) (Shapiro and Teukolsky 1983). We have made use of this approximation in conjunction with the hybrid equation of state described in $\S 4.1$ to show that our numerical model yields results that match those of Zwerger and Müller (1997).

However, nature is not this simple and detailed stellar evolution calculations (Woosley, Heger, and Weaver 2002) have shown that the iron cores of evolved massive stars are not perfect polytropes. Rather, they have a complicated thermodynamic and compositional structure. Fig. 1 depicts the differences between the density profiles of progenitor models from Woosley and Weaver (1995) and the density profile of the polytrope used by Zwerger and Müller (1997). In Fig. 2, we show the profiles of the electron fraction $\left(Y_{e}\right)$ of the Woosley and Weaver (1995) progenitors, and contrast them with the flat $Y_{e}=0.5$ profile of the Zwerger and Müller polytrope.

For our study we have used 11,15 , and $20 \mathrm{M}_{\odot}$ presupernova models from Woosley and Weaver (1995). As Figure 1 demonstrates, the pre-collapse core density and $Y_{e}$ profiles of the Woosley and Weaver (1995) 25 and $20 \mathrm{M}_{\odot}$ models are very similar. The temperature profiles also match. Thus, one would expect very similar collapse dynamics and gravitational wave signatures. A test calculation with the $25 \mathrm{M}_{\odot}$ progenitor bears out this expectation (Table 5).

\section{ROTATION}

Rotation is a key agent in the development of asphericity during core collapse and, hence, for the emission of gravitational radiation. Depending on the initial rotation rate and the angular momentum distribution, conservation of angular momentum may lead to very rapidly rotating compact remnants, which are unstable on secular, or even dynamical, timescales. In the approximation of MacLaurin spheroids (incompressible, uniform density, rigidly rotating equilibrium configurations), triaxial instabilities may grow if $\beta \geq 0.14$ and $\beta \geq 0.27$ for secular and dynamical instabilities, respectively (Tassoul 1978). Recently, Centrella et al. (2001) have shown that models with off-center density maxima (i.e. with a toroidal structure) can already become dynamically unstable at values of $\beta \geq 0.14$. However, the critical $\beta$ s required for either secular or dynamical instability were derived in the past using either constant-density models or very compact cores whose deviation from uniform density was modest. Recently, Shibata, Karino and Eriguchi (2003) have shown that rotating polytropes with centrally located density maxima can become dynamically unstable even for $\beta$ on the order of $1 \%$ if they are strongly differentially rotating. In this paper, we calculate the total $\beta$ s for an entire realistic iron core with a large dynamic range of densities ( $\sim$ eight orders of magnitude). This is not the $\beta$ for only the inner, more uniform, core. The outer regions of realistic iron cores do not move much during the crucial dynamical phases of the inner core important for the estimation of the gravitational wave signature. Importantly, the actual critical $\beta$ s necessary for triaxial deformation have yet to be determined for such structures. To our knowledge, all investigations to date of the growth of triaxial instabilities have lacked either realistic initial models or sophisticated equations of state. These limitations should be kept in mind when assessing previous work.

The centrifugal forces connected to rotation do not only cause asphericity, but slow down the core collapse and may, provided the configuration has the right angular momentum distribution, stop the collapse before nuclear matter density is reached ("subnuclear bounce"). A critical condition for the stabilizing effect of rotation on (pseudo-) radial modes of stars in the Newtonian regime is

$$
\Gamma>\Gamma_{\text {crit }}=\frac{2}{3} \frac{(2-5 \beta)}{(1-2 \beta)},
$$

where $\Gamma$ is the effective adiabatic index that describes the change of pressure along a collapse trajectory of a given mass element:

$$
\Gamma=\left.\frac{\partial \ln P}{\partial \ln \rho}\right|_{M}
$$

(Ledoux 1945; Tohline 1984; Mönchmeyer et al. 1991). Hence, for a given progenitor structure and equation of state with an effective adiabatic index $\Gamma$, there is a critical value of the rotation parameter above which the configuration is stable against collapse:

$$
\beta>\beta_{\text {crit }}=\frac{1}{2} \frac{(4-3 \Gamma)}{(5-3 \Gamma)} .
$$

It is known that massive stars on the main sequence rotate rapidly, with typical equatorial rotational velocities of $\sim 200 \mathrm{~km} \mathrm{~s}^{-1}$ (Fukuda 1982). This is a significant fraction of their breakup velocity. Unfortunately, since observations of the stellar surface tell us little about the angular momentum of the stellar interior (or its evolution), one has to rely on parameter dependent, semi-phenomenological prescriptions 
to follow these quantities numerically. Heger, Langer, and Woosley (2000) have built upon the one-dimensional calculations of Woosley and Weaver (1995) and incorporate a prescription for angular momentum transport. We include in our progenitor model suite their "rotating" 15 and $20 \mathrm{M}_{\odot}$ progenitor models. Heger et al. (2003) have extended the work of Heger et al. (2002) by the inclusion of the effects of magnetic torques on rotational evolution and provide corresponding cores for 15 , 20 and $25 \mathrm{M}_{\odot}$ models. Table 1 lists all the presupernova models we have employed in this study. Since the gravitational wave signature is sensitive to the distribution of angular momentum throughout the iron core, gravitational waves may eventually be used to learn about the interior rotational structure of massive stars.

We have used two different approaches to include rotation in our calculations. First, we follow Zwerger and Müller (1997) in forcing the one-dimensional initial models to rotate with constant angular velocity on cylinders on our axisymmetric grid according to the rotation law

$$
\Omega(r)=\Omega_{0}\left[1+\left(\frac{r}{\mathrm{~A}}\right)^{2}\right]^{-1},
$$

where $\Omega(r)$ is the angular velocity, $r$ is the distance from the rotation axis, and $\Omega_{0}$ and $\mathrm{A}$ are free parameters that determine the rotational speed/energy of the model and the distribution of angular momentum. Large values of A lead to very rigid rotation, small values to strongly differential rotation. Our parameter studies are performed over a range of $\beta_{i}$ and $\mathrm{A}$, where $\beta_{i}$ is the initial $\beta$ of the model. The choices for $\beta_{i}$ and $\mathrm{A}$ were based upon current knowledge of iron core rotation, but exclude strongly differential rotation (Heger et al. 2003; Heger, Langer, and Woosley 2000). Note that there has been some confusion in the literature concerning the meaning of $r$ in eq. 5. Mönchmeyer et al. (1991) and Kotake, Yamada, and Sato (2003) interpreted $r$ as radial distance from the origin, whereas Zwerger and Müller (1997) and Dimmelmeier, Font, and Müller (2002b) understood it as distance from the rotation axis. We follow the latter definition of $r$ as it accords with the Poincare-Wavre theorem which predicts that the specific angular momentum is constant on cylinders for degenerate rotating objects (for a review see Tassoul 1978).

We name our runs according to the following convention: [initial model name] $\mathrm{A}[\mathrm{in} \mathrm{km}] \beta_{i}[$ in \%]. For example, s11 A $1000 \beta 0.3$ is a Woosley and Weaver (1995) $11 \mathrm{M}_{\odot}$ model with $\mathrm{A}=1000 \mathrm{~km}$ and an initial $\beta_{i}$ of $0.3 \%$.

In contrast to Zwerger and Müller (1997), we do not use rotational equilibrium configurations, since these can only be found consistently for models with constant entropy and $Y_{e}$ (Hachisu 1986a). For direct comparisons with Zwerger and Müller (1997) we used models with small initial rotation rates $\left(\beta_{i}\right)$ in which the initial deformation due to rotation would be negligible. Zwerger and Müller (1997) found a maximum difference of $10 \%$ in the central densities at bounce for a strongly rotating model evolved with and one evolved without an initial rotational equilibrium configuration. Since the progenitor models with $\beta_{i} \gtrsim 1 \%$ tend not to collapse $\left(\beta_{i}>\beta_{\text {crit }}\right.$, if nothing artificial is done to alter their structure), we have limited our study to models with $\beta_{i} \leq 1 \%$. Hence, we expect the error that is introduced by the non-equilibrium rotational configuration at the onset of collapse to be very small and, in the worst case, to be on the order of a few percent. In addition to this, we point out that Zwerger and Müller (1997) argue that the use of non-equilibrium models is justified if the stellar core collapses slowly enough to allow for the adjustment to the appropriate angular density stratification for its rate of rotation. This is certainly the case for our models, which all collapse on a timescale on the order of 100-500 milliseconds (ms).

Secondly, we have made use of the recent presupernova models of Heger, Langer, and Woosley (2000) and Heger et al. (2003) that, though they are intrinsically one-dimensional, take into account the effects of centrifugal forces on the stellar structure before carbon burning ends. Furthermore, redistribution of angular momentum and chemical species were modeled using a set of prescriptions and assumptions for mixing and transport processes. In particular, all torques were assumed to lead to rigid rotation on some physical timescale (Fryer and Heger 2000). The "magnetic" models of Heger et al. (2003) assume a magnetic dynamo process that generates fields which inhibit differential rotation and lead to slower core rotation at collapse.

In Fig. 3, the profiles for selected models of the initial angular velocity versus radius are shown. Note that the differences due to different progenitor masses are negligible compared with the order-of-magnitude differences introduced by the inclusion of magnetic-field effects during stellar evolution. One should be cautious, however, in accepting these results since research on stellar evolution with rotation is still in its infancy.

\section{NUMERICAL TECHNIQUES}

\subsection{Equations of State}

For all our calculations involving realistic progenitor models we have made use of the equation of state of Lattimer and Swesty (1991) (the LSEOS). It is based on the finitetemperature liquid drop model of nuclei developed in Lattimer et al. (1985). Our particular implementation is the one presented in Thompson, Burrows, and Pinto (2003) that uses a three-dimensional table in temperature $(T)$, density $(\rho)$, and $Y_{e}$. At each point in the table the specific internal energy, the pressure $(P)$, the entropy per baryon $(s)$, and compositional information are stored. Using integer arithmetic to find nearest neighbor points for a given set of $\rho, T, Y_{e}$, the need for timeconsuming search algorithms has been eliminated. Given $\rho, T$ and $Y_{e}$, the code performs three six-point bivariant interpolations in the $T-\rho$ planes nearest to and bracketing the given $Y_{e}$ point. A quadratic interpolation is then executed between $Y_{e}$ points to obtain the desired thermodynamic quantity. Since our hydrodynamic routine updates specific internal energy, we employ a Newton-Raphson/bisection scheme which iterates on temperature at a fixed internal energy until the root is found to within a part in $10^{8}$.

The LSEOS extends down to only $\sim 5 \times 10^{6} \mathrm{~g} \mathrm{~cm}^{-3}$ and its validity in this density regime is guaranteed only for fairly high temperatures, where the assumption of nuclear statistical equilibrium (NSE) still holds. For calculations involving lower densities, Thompson, Burrows, and Pinto (2003) have coupled the LSEOS to the Helmholtz EOS (Timmes and Arnett 1999; Timmes and Swesty 2000), which contains electrons and positrons at arbitrary degeneracy and relativity, photons, nuclei and nucleons as non-relativistic ideal gases, and Coulomb corrections. 
To facilitate the comparison of our results with those of Zwerger and Müller (1997) and Dimmelmeier, Font, and Müller (2002b), we have implemented the "hybrid" equation of state used in those studies of collapsing $\Gamma=4 / 3(n=3)$ polytropes (Janka, Zwerger, and Mönchmeyer 1993). It consists of a polytropic part $P_{p}$ and a thermal contribution $P_{t h}$

$$
P=P_{p}+P_{t h} \text {. }
$$

The thermal part accounts for the thermal pressure of the highentropy material heated by the bounce shock and is given by

$$
P_{t h}=\left(\Gamma_{t h}-1\right) u_{t h},
$$

where $\Gamma_{t h}$ is set to 1.5 . The thermal energy density $u_{t h}$ is given by the total energy density $u$ through the relation

$$
u=u_{p}+u_{t h}
$$

where $u_{p}$ is the energy density of the degenerate electron gas.

The polytropic part

$$
P_{p}=K \rho^{\Gamma}
$$

reflects the pressure contributions due to the degenerate and relativistic electrons and (in the regime of nuclear density) the repulsive action of nuclear forces. The polytropic constant is initially set to

$$
K=\frac{3}{4}\left(\frac{\pi}{3}\right)^{2 / 3} \hbar c\left(\frac{Y_{e}}{m_{B}}\right)^{4 / 3}=1.2435 \times 10^{15} Y_{e}^{4 / 3} c g s,
$$

where $Y_{e}$ is the electron number fraction and the other quantities have their usual meaning (Shapiro and Teukolsky 1983). $\Gamma$ is chosen to be 1.32 to initiate the collapse of the $n=3$ polytrope. $Y_{e}$ is set to 0.5 . To mimic the stiffening of the equation of state at nuclear density ( $s e t$ to $\rho_{n u c}=2.0 \cdot 10^{14} \mathrm{~g} \mathrm{~cm}^{-3}$ ), $\Gamma$ is for $\rho \geq \rho_{\text {nuc }}$ set to 2.5 and $K$ is modified by the requirement of continuity of the thermodynamic variables at $\rho_{n u c}$ (Janka, Zwerger, and Mönchmeyer 1993).

\subsection{Hydrodynamics - VULCAN/2D}

Our simulations were performed with the Newtonian twodimensional finite-volume hydrodynamic code VULCAN/2D developed by Eli Livne (Livne 1993). VULCAN/2D uses a scalar von Neumann-Richtmyer artificial viscosity scheme for shock handling. The hydrodynamic equations are solved in the Lagrangian formulation and the hydrodynamical data are remapped after each time step onto a fixed Eulerian grid. VULCAN/2D can be run in implicit or explicit time integration mode. Since we are dealing with supersonic flows, we use VULCAN/2D in explicit mode. VULCAN/2D is second-order accurate in time and space and has been rigorously tested and compared with one-dimensional Lagrangian and Eulerian hydrodynamic codes (Livne 1993).

A feature of VULCAN/2D is its ability to deal with arbitrarily shaped grids while using cylindrical coordinates. For the problem of stellar collapse we have chosen a polar grid with logarithmic spacing for the region outside the inner $10 \mathrm{~km}$ and an inner Cartesian grid (see Fig. 4). In this way we circumvent severe time step constraints that would be imposed in the angular direction in the central region of a regular polar grid. The price we pay for this is the "horns" seen in Fig. 4 in the central region which are a consequence of the demand for continuity at the boundary between the central and the outer grid.

For our production runs we use 412 radial and 91 angular zones (including the central region), encompassing 1500 to $3000 \mathrm{~km}$ in radial extent (depending on the initial model) and covering the full 180 degrees of the symmetry domain. In our comparisons with Zwerger and Müller (1997), this resolution has been shown to reproduce their results to better than $10 \%$.

\subsection{Gravitational Wave Signature Extraction and Waveforms}

We have calculated the gravitational wave field in the slowmotion, weak-field quadrupole approximation (Misner, Thorne, and Wheeler 1973). The dimensionless gravitational wave strain $h$ is

$$
h_{i j}^{T T}(\overrightarrow{\mathbf{D}}, t)=\frac{2 G}{D c^{4}} \ddot{I}{ }_{i j}^{T T}\left(t-\frac{D}{c}\right),
$$

where $D=|\overrightarrow{\mathbf{D}}|$ is the distance between the observer and the source and

$$
\Psi_{i j}^{T T}=P_{i j k l}(\overrightarrow{\mathbf{N}}) \int d^{3} \mathbf{x} \rho\left[x_{k} x_{l}-\frac{1}{3} \delta_{i j} x_{m} x^{m}\right]
$$

is the transverse-traceless part of the reduced Cartesian massquadrupole tensor. $P_{i j k l}(\overrightarrow{\mathbf{N}})$ (with $\overrightarrow{\mathbf{N}}=\overrightarrow{\mathbf{D}} / D$ ) is the transversetraceless (TT) projection operator onto the plane orthogonal to the outgoing wave direction $\mathbf{N}$ and is of the form:

$$
\begin{array}{r}
P_{i j k l}(\overrightarrow{\mathbf{N}})=\left(\delta_{i k}-N_{i} N_{k}\right)\left(\delta_{j l}-N_{j} N_{l}\right) \\
-\frac{1}{2}\left(\delta_{i j}-N_{i} N_{j}\right)\left(\delta_{k l}-N_{k} N_{l}\right) .
\end{array}
$$

Direct application of eq. (11) (known in the literature as the "standard quadrupole formula" (SQF)) in a numerical fluid dynamics calculation is problematic, since numerically troublesome second time derivatives of the quadrupole moment are involved and the moment arm emphasizes contributions of lowdensity material far from the central regions (Finn and Evans 1990).

Using the Euler equations of inviscid hydrodynamics, Finn and Evans (1990), Nakamura and Oohara (1989), and Blanchet, Damour, and Schäfer (1990) derived formulations of the quadrupole formula involving either only one time derivative and easier, more tractable, spatial derivatives or spatial derivatives of the hydrodynamic observables exclusively. We use the formulation of Nakamura and Oohara (1989) and Blanchet, Damour, and Schäfer (1990):

$$
\begin{array}{r}
h_{i j}^{T T}(\overrightarrow{\mathbf{D}}, t)=\frac{2 G}{D c^{4}} P_{i j k l}(\overrightarrow{\mathbf{N}}) \times \\
\int d^{3} \mathbf{x} \rho\left[2 v^{k} v^{l}-x^{k} \partial_{l} \Phi-x^{l} \partial_{k} \Phi\right]
\end{array}
$$

where $\Phi$ is the Newtonian gravitational potential, $\rho$ is the massdensity, and $v$ the velocity.

For our 2-dimensional axisymmetric calculations, it is useful to rewrite the full gravitational radiation field in terms of the "pure-spin tensor harmonics" $T_{i j}^{E 2, l m}$ and $T_{i j}^{B 2, l m}$ (Thorne 1980; Mönchmeyer et al. 1991):

$$
\begin{aligned}
& \tilde{h}_{i j}^{T T}(\overrightarrow{\mathbf{D}}, t)=\frac{1}{D} \sum_{l=2}^{\infty} \sum_{m=-l}^{l} {\left[A_{l m}^{E 2}\left(t-\frac{D}{c}\right) T_{i j}^{E 2, l m}(\theta, \phi)+\right.} \\
&\left.A_{l m}^{M 2}\left(t-\frac{D}{c}\right) T_{i j}^{M 2, l m}(\theta, \phi)\right] .
\end{aligned}
$$

The coefficients $A_{l m}^{E 2}$ and $A_{l m}^{M 2}$ represent the mass quadrupole and the mass-current quadrupole contributions, respectively. In 
the quadrupole approximation, higher-order as well as masscurrent contributions are neglected and due to the assumption of axisymmetry only one non-vanishing term remains in eq. (15), namely $A_{20}^{E 2}$. By comparing eq. (14) with the lowest-order term of eq. (15), Mönchmeyer et al. (1991) write $A_{20}^{E 2}$ in terms of the hydrodynamic variables:

$$
\begin{array}{r}
A_{20}^{E 2}=\frac{16 \pi^{3 / 2}}{\sqrt{15}} \frac{G}{c^{4}} \int_{-1}^{1} \int_{0}^{\infty} r^{2} d \mu d r \cdot \rho . \\
{\left[v_{r}^{2}\left(3 \mu^{2}-1\right)+v_{\theta}^{2}\left(2-3 \mu^{2}\right)-v_{\phi}^{2}-6 v_{r} v_{\theta} \mu \sqrt{1-\mu^{2}}\right.} \\
\left.-r \partial_{r} \Phi\left(3 \mu^{2}-1\right)+3 \partial_{\theta} \Phi \mu \sqrt{1-\mu^{2}}\right],
\end{array}
$$

where $\mu=\cos \theta$ and $v_{r}, v_{\theta}$, and $v_{\phi}$ are the components of the velocity vector in the $r, \theta$, and $\phi$ directions. Furthermore, $\partial_{r}=\partial / \partial r$ and $\partial_{\theta}=\partial / \partial \theta$. The components of the approximate gravitational wave field $h^{T T}$ are then given by (Thorne 1980; Mönchmeyer et al. 1991):

$$
h_{\theta \theta}^{T T}=\frac{1}{8} \sqrt{\frac{15}{\pi}} \sin ^{2} \alpha \frac{A_{20}^{E 2}}{D} \equiv h_{+},
$$

where $\alpha$ is the angle between the symmetry axis and the line of sight of the observer. The only other nonzero component is $h_{\phi \phi}^{T T}=-h_{\theta \theta}^{T T}=-h_{+} . h_{\times}$equals zero, due to the assumption of axisymmetry. $h_{+}$and $h_{\times}$are the dimensionless wave strains corresponding to the two independent polarizations of the gravitational radiation field (Misner, Thorne, and Wheeler 1973).

The total gravitational energy radiated over time is given by

$$
E_{G W}=\frac{c^{3}}{32 \pi G} \int_{-\infty}^{\infty}\left|\frac{d A_{20}^{E 2}}{d t}\right|^{2} d t
$$

As an alternative to eqs. (16) and (17), we also implemented the "first moment of momentum divergence" formula of Finn and Evans (1990) (eq. 38 of their paper):

$$
\begin{aligned}
\frac{d I_{z z}}{d t}= & \frac{4 \pi}{3} \int_{-1}^{1} d \mu \int_{0}^{\infty} d r r^{3} \rho \times \\
& {\left[P_{2}(\mu) v_{r}+\frac{1}{2} \frac{\partial P_{2}(\mu)}{\partial \theta} v_{\theta}\right], }
\end{aligned}
$$

where $P_{2}(\mu)$ is the second Legendre polynomial in $\mu$ and $I_{z z}$ is the zz-component of the reduced mass-quadrupole moment tensor. The gravitational wave strain is then obtained through

$$
h_{\theta \theta}^{T T}=\frac{6 G}{D c^{4}} \sin ^{2} \alpha \frac{d^{2}}{d t^{2}} I_{z z} \text {. }
$$

\subsubsection{Energy Spectra}

Writing $A_{20}^{E 2}$ in eq. (18) in terms of the inverse Fourier transform

$$
A_{20}^{E 2}(t)=\int_{-\infty}^{\infty} \tilde{A}_{20}^{E 2}(f) e^{-2 \pi i f t} d t
$$

and after several algebraic steps, we obtain:

$$
\frac{d E(f)}{d f}=\frac{c^{3}}{G} \frac{(2 \pi f)^{2}}{16 \pi}\left|\tilde{A}_{20}^{E 2}(f)\right|^{2}
$$

in terms of the Fourier transform

$$
\tilde{A}_{20}^{E 2}(f)=\int_{-\infty}^{\infty} A_{20}^{E 2}(t) e^{2 \pi i f t} d f .
$$

The total radiated energy is then obtained from the integral over the energy spectrum

$$
E_{G W}=\int_{0}^{\infty} \frac{d E(f)}{d f} d f
$$

which should be identical to the result obtained from eq. (18).

We have implemented eq. (22) using the Fast Fourier Transform (FFT) technique (Press et al. 1992). Since the wave amplitude is calculated at unequal time intervals due to variations in the time step, we first interpolate the data onto an evenlyspaced temporal grid before applying the FFT. We have verified that the value of $E_{G W}$ obtained using eq. (18) is always within a few percent of that obtained using eq. (24).

\section{METHOD VALIDATION - POLYTROPES}

Table 2 summarizes the results of our comparisons with the work of Zwerger and Müller (1997), who used simple $n=3$ polytropes and the hybrid EOS discussed in §4.1. Our results match those of Zwerger and Müller (1997) in density at bounce $\left(\rho_{\max }\right)$, maximum gravitational wave amplitude $\left(A_{20}^{E 2} \max \right)$ and total gravitational wave energy $\left(E_{G W}\right)$, in most cases to better than $10 \%$. The largest difference in maximum density is $22 \%$ and is found in model A500 $\beta 0.9$ (Zwerger and Müller's A3B3G2). However, Dimmelmeier, Font, and Müller (2002b) performed a similar comparison study with their code and obtained for this model a density at bounce much closer to ours. As to $E_{G W}$, our models generally yield larger values than those of Zwerger and Müller (1997), since ours were evolved for longer periods of time after bounce, thus tracking a greater part of the post-bounce aspherical motion of the compact remnant.

Using the publicly available data of the Newtonian runs of Dimmelmeier, Font, and Müller (2002b), we find that our waveforms match perfectly up to bounce and then start to exhibit a slight shift in post-bounce maxima, minima and periods. We attribute these differences in part to the fact that our artificial viscosity scheme for hydrodynamic shock capture leads to slightly greater artificial damping than the piecewise parabolic method (PPM) used in the cited work and to our simulation on the full $180^{\circ}$ domain.

\section{RESULTS USING REALISTIC PROGENITOR MODELS}

Collapse dynamics of rotating supernova progenitors is governed by three major forces: gravity, pressure gradients, and centrifugal forces. In the canonical model of non-rotating core collapse, photo-dissociation of iron peak nuclei and electron capture on nuclei and free protons initiate core collapse. As core collapse progresses, an almost homologously $(v \propto \mathrm{r})$ collapsing central region, the inner core, forms, while the outer core collapses supersonically. With increasing density, electron capture rates grow until a density of about $3 \times 10^{12} \mathrm{~g} \mathrm{~cm}^{-3}$ is reached, at which time the matter becomes opaque to the electron capture neutrinos which are then trapped in the core. As nuclear density is approached, nuclear repulsive forces lead to a sudden stiffening of the equation of state, initiating the bounce of the inner core and the subsequent outward propagation of the bounce shock.

When rotation is included, centrifugal forces counteracting gravity's pull change the dynamics of collapse. Depending on the total amount of angular momentum and its distribution throughout the collapsing core, the effects can be either minor, leading only to small deformation (oblateness) of the core 
and bounce at slightly lower maximum densities or - if there is a great amount of angular momentum - major, resulting in "fizzlers" with large deformations and slower bounces at subnuclear densities (Shapiro and Lightman 1976; Tohline 1984). Mönchmeyer et al. (1991) give a detailed description of the hydrodynamics of rotational core collapse. Since this paper is primarily concerned with the gravitational wave signature of core collapse, in the following we provide only brief descriptions of the most salient features of the hydrodynamics.

\subsection{Hydrodynamical Evolution and Gravitational Wave Signature}

We separate our models into two types. Type I encompasses those models that experience core bounce predominantly due to the stiffening of the nuclear equation of state at or above nuclear densities and type II comprises models that bounce due to centrifugal forces. We refer to those models that experience significant centrifugal forces, but still bounce at or close to nuclear densities, as type I/II. We show that types I and II have distinctive and characteristic gravitational wave signatures, while the wave signature of an type I/II exhibits a mixture of type I and type II features. Zwerger and Müller (1997) introduced a similar classification scheme. We, however, do not see the behavior which they call type III since its occurence is connected with very low effective $\Gamma$ s and extremely rapid collapsei, which none of our models shows.

We first present the systematics with $\beta_{i}$ that we have found for the s15 model from Woosley and Weaver (1995) with the parameter A of the rotation law (eq. 5) set to $1000 \mathrm{~km} . \Omega_{0}$ is adjusted to yield the wanted value of $\beta_{i}$. Recall that our $\beta$ is calculated for the entire iron core with its many decades of density, and not just for the inner or homologously collapsing region. In $\S 6.2$ and $\S 6.3$ we discuss the differences introduced by different choices for A and initial model. Finally, in $\$ 6.4$, we present the results we obtained from core collapse calculations with the rotating progenitor models from Heger, Langer, and Woosley (2000) and Heger et al. (2003).

Type I: Models of type I rotate so slowly that centrifugal forces are not able to stop collapse before nuclear densities are reached. The central region of the stellar core plunges deeply into the potential well and quickly and significantly overshoots to supranuclear densities before its infall velocities are reversed on a timescale of less than a millisecond by the solid-walllike action of the repulsive nuclear forces. As in nonrotating models, a strong shock forms at the boundary between the subsonically collapsing inner core and the supersonically infalling outer mantle.

Due to angular momentum conservation and, hence, the growing influence of centrifugal forces with increasing compactness, the core bounce is not spherically symmetric, but happens first and most strongly at the poles. Hence, the strong bounce shock is aspherical, propagating faster along the poles.

In type I models, the initial oblateness of the core at bounce becomes an oblate-prolate $(l=2)$ oscillation, accompanied by higher-order modes, in addition to the already present postbounce radial "ringing" of the compact remnant. This is seen in Fig. 5, which shows the innermost $200 \mathrm{~km}$ by $200 \mathrm{~km}$ of the collapsed core of model s15A1000 $\beta 0.2$ (type I). Shortly after bounce and after the shock wave is launched, the core oscillates wildly in a superposition of modes that are damped on timescales of milliseconds, since oscillation leads to dissipation by the periodic emission of strong sound waves. As Fig. 5 indicates, the amplitude of the $l=2$ deformation has decayed considerably $13 \mathrm{~ms}$ after bounce, at which time predominantly vortical and incoherent motions have emerged to dominate.

Figure 6 portrays the evolution of the maximum density of the s15A1000 model series with $A=1000 \mathrm{~km}$. Plotted with a dashed line is the evolution of the maximum density of a nonrotating model. The models with $\beta_{i}=0.10 \%$ and $\beta_{i}=0.20 \%$ bounce at or very close to nuclear density and exhibit type I model behavior. The $\beta_{i}=0.30 \%$ model, however, though experiencing bounce at about nuclear density, exhibits one cycle of pronounced large-scale coherent radial expansion and re-contraction, followed by a second, but weaker bounce after which the volume mode is quickly damped due to the formation of a second shock wave. We refer in the following to this cycle of expansion, re-contraction and secondary bounce as the "expansion-collapse-bounce cycle." It marks the beginning of the transition from type I to type II behavior with increasing $\beta_{i}$.

The evolution of the rotation parameter $\beta=E_{\text {rot }} /\left|E_{\text {grav }}\right|$, an integral quantity and a global measure of the system, is shown in Fig. 7. For models that show clean type I behavior, namely those with $\beta_{i}$ less than $0.30 \%, \beta(\mathrm{t})$ manifests only one notable post-bounce minimum, directly paralleling the evolution of the maxmium density. The first transition model with $\beta_{i}=0.30 \%$ reaches a $\beta$ that is $30 \%$ higher at bounce and exhibits multiple post-bounce minima and maxima before reaching its final value of $6.37 \%$, the largest in the s15A1000 model series.

We now focus on the characteristics of the gravitational wave signature of a type I model and see how the signature changes with increasing $\beta_{i}$. In the quadrupole approximation that we made in $\$ 4.3$, the amplitude of the gravitational waves is directly connected to the second time derivative of the reduced mass-quadrupole moment $\left(t_{z z}\right.$; eq. 12). Figures $8 \mathrm{a}$ and $8 \mathrm{~b}$ show the evolution of the maximum density and the quadrupole gravitational wave amplitude for times shortly before and after bounce of the typical type I models s15A1000 $\beta 0.1$ and s15A1000 $\beta 0.2$. From the way $t_{z z}$ evolves, we can divide the evolution of the wave signal into 3 phases (Mönchmeyer et al. 1991).

Phase 1 (the "infall phase") coincides with the hydrodynamic collapse phase, and is marked by the accelerated increase (in absolute value) of the quadrupole moment as angular momentum conservation forces the collapsing core to deviate more and more from spherical symmetry. When the core approaches bounce, the increase of $\left|I_{z z}\right|$ decelerates (i.e., the wave signal becomes negative) and eventually is reversed into a decrease of $\left|I_{z z}\right|$, the smaller core size counteracting the effect of the increasing density on the quadrupole moment. This marks the transition to phase 2 (the "bounce phase"). The wave signal reaches its absolute minimum a fraction of a millisecond after core bounce and then increases, reaching positive values during the first post-bounce local minimum of the central density. As said, right before and during core bounce, the increase of $\left|I_{z z}\right|$ started in phase 1 is dramatically reversed. Nevertheless, the first time derivative of $I_{z z}$ reaches positive values for only a short period and $I_{z z}$ itself remains negative throughout the entire evolution, increasing again in absolute value after bounce. Finally, phase 3 (the "ring-down phase") is characterized by small amplitude oscillations of the wave signal that reflect the core ring-down occurring in type I models. 
The three phases that we described in the preceding paragraph are easily discernible in Figs. 8a and 8b. As centrifugal forces become increasingly significant with larger $\beta_{i}$, the evolution of the maximum density and of the waveform itself change. Figure $8 \mathrm{c}$, depicting model s15A1000 $\beta 0.3$, shows this well. This model undergoes one large post-bounce oscillation, an "expansion-collapse-bounce cycle." A second, less strong, bounce occurs and is reflected in a second pronounced spike in the waveform. Increasing $\beta_{i}$ to $0.40 \%$ changes the dynamics drastically to what we classify as type II behavior.

Type II: Models of type II experience bounces at subnuclear densities caused by centrifugal forces. Whereas core bounce due to repulsive nuclear forces results in an abrupt reversal of the quasi-homologously collapsing inner core on a timescale of less than $1 \mathrm{~ms}$, a bounce due to the action of centrifugal forces occurs on longer timescales and with smaller accelerations. This can be understood from the fact that rotation acts approximately like a gas with a $\Gamma$ of 5/3 (Tassoul 1978; Mönchmeyer et al. 1991). Fig. 6 depicts the evolution of the maximum density of models with $\beta_{i}$ s from $0.0 \%$ to $1.00 \%$. For $\beta_{i} \gtrsim 0.4 \%$, the models exhibit type II behavior.

With increasing $\beta_{i}$, the density at bounce decreases, the maxima become much wider, and the accelerations grow smaller. The rotation-dominated, highly aspherical bounce of type II models happens at larger radii and creates, since it is much less abrupt, less entropy and much weaker bounce shocks than in type I models. After core bounce, the core expands coherently, leading to an almost order of magnitude drop in the maximum density. This expansion is reversed when gravitational forces again begin to dominate over pressure gradients and centrifugal forces. In this way, the quickly spinning core undergoes several damped expansion-collapse-bounce cycles until it settles into an equilibrium configuration. In fact, it acts much like a damped harmonic oscillator with a Hamiltonian consisting of radial kinetic, rotational, internal, and gravitational energies (Mönchmeyer et al. 1991).

Figures $8 d$ and 9a through $9 \mathrm{~d}$ depict the time evolution of the maximum density and of the quadrupole gravitational wave amplitude for the type II models of the s15A1000 model series. For these models, the "ring-down" signature seen in type I models is replaced by the signature of coherent expansioncollapse-bounce cycles, manifest by wide peaks in the wave signal. Model s15A1000 $\beta 0.4$ (Fig. 8d) still shows shorter period substructure, originating from additional nonspherical pulsation modes, but such substructure fades away with larger $\beta_{i}$. The absolute values of the gravitational wave amplitude peaks are smaller than those of type I models, since deceleration and acceleration occur over longer timescales. This is also reflected in the longer characteristic periods and in the lower characteristic frequencies of the gravitational wave signal.

The gravitational wave energy spectra (eq. 22) for a characteristic subset of the s15A1000 models are shown in Fig. 10. Since the gravitational wave luminosity goes with the slope of the waveform, most of the energy is radiated for all models in the spike connected to the first bounce. We associate the dominant frequencies of the gravitational wave energy spectra with this first spike of the waveform. In Table 4, we summarize the results from all our calculations involving the s15 progenitor model. Tables 3, 5 and 6 contain the quantitative results of calculations using the s11 and s20 models of Woosley and Weaver (1995) and the models of Heger, Langer, and Woosley (2000) and Heger et al. (2003).

The spectrum of s15A1000 $\beta 0.2$, which shows type I behavior, is dominated by frequencies between $300 \mathrm{~Hz}$ and $600 \mathrm{~Hz}$ and peaks at $460 \mathrm{~Hz}$. Most of the smaller peaks are connected to the first spike in the waveform during which $94 \%$ of the total gravitational wave energy of this model is radiated (Table 4). There is, however, a contribution by the radial and nonradial ring-down pulsations that have characteristic periods of 2 $-2.5 \mathrm{~ms}$ in this model, translating to frequencies of $400-500 \mathrm{~Hz}$. The peak at $700 \mathrm{~Hz}$ and the one-order-of-magnitude-suppressed peak at about $1400 \mathrm{~Hz}$ are higher harmonics of lower frequency contributions, as well as higher frequency modes of the ringdown oscillation. With increasing $\beta_{i}$ the spectrum shifts to lower frequencies and lower absolute values, peaking at 152 $\mathrm{Hz}\left(\beta_{i}=0.40 \%\right), 91 \mathrm{~Hz}\left(\beta_{i}=0.60 \%\right)$, and $38 \mathrm{~Hz}\left(\beta_{i}=0.80 \%\right)$. Furthermore, a prominent peak at low frequencies (in this series beginning with model s15A1000 $\beta 0.4$ ) can be directly associated with the oscillation frequency of the post bounce expansioncollapse-bounce cycles seen in type II models.

As shown in Fig. 10, the energy spectra provide an excellent way to learn about overall collapse dynamics and clearly exhibit the great changes brought about by even moderate rotation. We now summarize the most important overall characteristics of the dynamical types: Type $\mathbf{I}$ is characterized by bounce at supranuclear densities and exhibits a clear subdivision into infall (1), bounce (2) and ring-down phases (3). The gravitational wave energy spectra of type I models peak above $350 \mathrm{~Hz}$ and the integrated radiated energy achieves its maximum, reaching $10^{-8} \mathrm{M}_{\odot} c^{2}$ for the fastest rotating model that still bounces at supranuclear density. On the other hand, type II models bounce at subnuclear densities due to the influence of centrifugal forces, exhibit much longer timescales, and manifest several harmonic oscillator-like expansion-collapse-bounce cycles. Their spectra peak at lower frequencies and the total energies radiated are, for the fastest rotators, one to two orders of magnitude smaller than those for type I (see Tables 3-6).

We must point out that the specific value of $\beta_{i}$ needed to produce a given type (I or II) varies with the degree of differential rotation and (more strongly) with the effective $\Gamma(\S 3)$, determined by the equation of state in combination with the microphysical processes occuring during collapse (e.g. electron capture, leading to a lower effective $\Gamma$ ). Moreover, the maximum value of $\beta_{i}$ needed to maintain type I behavior, depends on the effective $\Gamma$ through eq. (4). Note that the final $\beta$ s achieved in our calculations are all below $10 \%$.

Since we have not included radiative transfer, our results should be considered preliminary. Furthermore, the collapse will depend on the rotation law used and on general relativistic effects. Hence, we cannot say, though the results of this study suggest, that a $\beta$ high enough for dynamical or secular instability might not occur during realistic stellar core collapse.

\subsection{Variation with Degree of Differential Rotation}

We have performed calculations with three different values of the parameter A of the rotation law described in $\$ 3$. To achieve rigid rotation throughout the entire core, we set A to $50000 \mathrm{~km}$ in eq. 5 and to explore moderate differential rotation, we use $A=1000 \mathrm{~km}$ and $A=500 \mathrm{~km}$. Figure 3 shows a sample of the rotation laws for different values of $\mathrm{A}$. 
In Fig. 11, we present results from our study of the A dependence of the gravitational wave signature for $\beta_{i}=0.5 \%$ and the s15 progenitor. Generally, as A is lowered, more rotational energy is (for a fixed $\beta_{i}$ ) moved interior to A. Hence, the influence of rotation on the collapsing central regions is larger in those models with smaller $\mathrm{A}$ and the transition from type I to type II behavior occurs for smaller $\beta_{i}$. This is illustrated in Fig. $11 \mathrm{c}$ where model s15A50000 $\beta_{i} 0.5$ still shows type I behavior, even though models s15A1000 and s15A500 have transitioned to type II. The same effect is seen in Fig. 11a. The energy spectra in Fig. 11b show low-frequency peaks at the frequencies of the expansion-collapse-bounce cycles for models s15A500 and s15A1000, while the spectrum of the s15A50000 model is still type I.

As indicated, for larger values of A, i.e. for a more rigidly rotating configuration, the value of $\beta_{i}$ must be higher for type II behavior to occur. This shifts to higher values the $\beta_{i}$ s required for maximum gravitational wave amplitudes and final $\beta$ s. This A dependence is generic and translates into corresponding behavior for s11 and s20 progenitors (see Tables 3, 4, and 5).

\subsection{Initial Model Comparisons}

To investigate the effect of changes in presupernova stellar structure on the collapse dynamics and, ultimately, on the gravitational wave signature, we have performed calculations using the s11, s15 and s20 progenitor models of Woosley and Weaver (1995) at given values of $\mathrm{A}$ and $\beta_{i}$. We choose $\mathrm{A}=1000 \mathrm{~km}$ and $\beta_{i}=0.5 \%$ for our progenitor model comparisons.

Initially, the s11 and s15 models are quite similar in their structures (see Figs. 1 and 2) and this similarity persists throughout their evolution. The s20 model, however, has a smaller initial central density, a larger $Y_{e}$, and a significantly larger iron core, encompassing about $1.7 \mathrm{M}_{\odot}$ and reaching out to $2200 \mathrm{~km}$ (Table 1).

Figure 12 displays the evolution of the maximum density of models s11A1000 $\beta 0.5$, s15A1000 $\beta 0.5$, and s20A1000 $\beta 0.5$. The s15 model reaches the highest densities and bounces due to centrifugal forces. After bounce, the s15 core executes the typical damped expansion-collapse-bounce cycles, as does model s11. However, model s20, with a significantly different initial structure, reaches lower densities than the s11 and s15 models and exhibits shorter periods in its post-bounce oscillations. Furthermore, its post-bounce oscillations are more quickly damped by the proximity of its stalled shock. A shock acts like a strong absorber of sound waves. As a result, the characteristic time for the purely hydrodynamic damping of inner-core oscillations is roughly the round-trip sound travel time to the shock radius. The larger this radius, the weaker the damping. The 2-dimensional plots (Fig. 13) of the specific entropy of model s20A1000 $\beta 0.3$ reveal some of the dynamics of the s20 models. As in s11 and s15 models with rotation, the shock forms first at the poles and propagates out faster along the rotation axis than at the equator. This is shown in the transition from Fig. 13a to Fig. 13b, in which the axis ratio is almost 2:1 and the shock has a prolate shape. Despite centrifugal forces, Fig. 13c shows that matter can flow in at the equator. The bounce shock stalls, but still executes oscillations in radius. We note that equatorial symmetry is clearly broken in Fig. 13d. This emphasizes the importance of including all $180^{\circ}$ in an axisymmetric simulation.
Figure 14 shows the waveforms of models s11A1000, $\mathrm{s} 15 \mathrm{~A} 1000$, and s20A1000 for $\beta_{i}=0.5 \%$. Models s 11 and s 15 exhibit clear type II behavior, with the variation in their wave forms paralleling that of their maximum densities. The wave form of the s20 model, however, exhibits different behavior; the stall of its bounce shock and subsequent accumulation of outercore material onto the compact remnant introduce additional higher-frequency components. As Fig. 15 indicates, this is also reflected in its gravitational wave energy spectrum, which contains more power at higher frequencies.

The bounce shock stalls in all our models with the s20 progenitor. For larger $\beta_{i}$, standard type II behavior is altered, since the post-bounce expansion-collapse-cycles are strongly damped by the stalled shock. We point out that to date the effect of a stalled shock upon the gravitational waves signature from rotating stellar collapse has not been discussed. However, such effects might be common for core-collapse supernovae, since in realistic models energy losses due to neutrinos - at least initially - lead generically to stalled bounce shocks.

\subsection{The Heger Models}

We have chosen the e15 and e20 models of Heger, Langer, and Woosley (2000) and the $\mathrm{m} 15 \mathrm{~b} 4, \mathrm{~m} 20 \mathrm{~b} 4$, and $\mathrm{m} 25 \mathrm{~b} 4$ models of Heger et al. (2003) for our study of progenitors evolved with rotation and magnetic fields ( $\$ 3$ ). Models from Heger, Langer, and Woosley (2000) that end with the letter 'b' (with $\beta_{i} \geq 3 \%$ ) were not used, since they undergo expansion and not collapse once mapped onto our two-dimensional grid. We would have had to artificially force these models to collapse by altering their thermodynamic structure. We have chosen not to do so and postpone the investigation of the gravitational wave signature from these models to future research that will include detailed weak interaction physics and neutrino transport.

The collapse, bounce, and post-bounce behavior up to about $100 \mathrm{~ms}$ after bounce of the e 15 model $\left(\beta_{i}=0.645 \%\right)$ conforms to type II behavior. Figure 16a shows the evolution of the maximum density and the gravitational waveform of model e 15 and Fig. 16b shows the evolution of these quantities for model s15A1000 $\beta 0.7$. Even though they experience core bounce at the same density, the period of the post-bounce oscillations of model e 15 is significantly shorter than that of the s15 model. Since shorter periods mean greater accelerations, the individual peaks of the waveform, associated with the first and subsequent bounces of the e 15 model, are greater than those of the s15 model. The same holds for the integrated radiated energy.

As with the s20 model, the bounce shock of the e15 model stalls, damping the type II-like post-bounce expansioncollapse-bounce cycles. This process introduces high frequency components into the waveform and, thus, into the energy spectrum of the gravitational wave signal (Fig. 16d). The e20 model has a lower initial $\beta_{i}$ than e15 and similar total angular momentum. Hence, it reaches a larger density at bounce and its gravitational wave energy spectrum peaks at about $193 \mathrm{~Hz}$ (for $\mathrm{e} 15$, it is $90 \mathrm{~Hz}$ ). However, the overall behavior is similar and the bounce shock of the e20 model also stalls. The subsequent infall of matter onto the inner core is non-spherical (see, for example, Fig. 13 and Table 6).

The models $\mathrm{m} 15 \mathrm{~b} 4, \mathrm{~m} 20 \mathrm{~b} 4$ and $\mathrm{m} 25 \mathrm{~b} 4$ have initial rotation parameter $\beta_{i}=E_{\text {rot }} /\left|E_{\text {grav }}\right|$ between $0.002 \%$ and $0.005 \%$. These 
values of $\beta_{i}$ are much smaller than the ones we investigated with the models of Woosley and Weaver (1995). The effects of rotation on the m-models are minimal and, as can be seen in Fig. 17 for models $\mathrm{m} 15 \mathrm{~b} 4$ and $\mathrm{m} 20 \mathrm{~b} 4$ (m25b4 is similar), the gravitational wave amplitudes from the bounce and ringdown phases are one to two orders of magnitude smaller than those of any s11/s $15 / \mathrm{s} 20$ model with $\beta_{i}=0.1 \%$ (see Fig. 8). In fact, as in the nonrotating case discussed below (\$6.5), most of the gravitational wave energy is being emitted by aspherical convective overturn after bounce (Table 6) and the spectra are dominated by the characteristic frequencies of the convective motions (Burrows and Hayes 1996; Müller and Janka 1997).

\subsection{Nonrotating Models}

As calibration, we performed calculations without rotation with the s11, s15 and s20 progenitor models from Woosley and Weaver (1995). Figure 18 shows the evolution of the maximum density and the gravitational wave amplitude for a nonrotating $\mathrm{s} 15$ model. The negative spike in the gravitational wave amplitude associated with core bounce exhibited by this model is about two orders of magnitude weaker than the one seen for models with slow rotation (Table 4). This is a consequence of the much smaller reduced mass-quadrupole moment of this nonrotating model, not deformed by centrifugal forces. Even though the collapse proceeds spherically, its reduced mass-quadrupole moment is initially not exactly zero, since small perturbations introduced by the numerical scheme and by the mapping of the one-dimensional progenitor model onto the two-dimensional computational grid give rise to some initial asphericity which grows during collaps.

After core bounce convective instability leads to aspherical bulk mass motion in the central regions, emitting small amplitude gravitational waves with frequencies corresponding to the characteristic turnover periods. Since this type of gravitational wave emission is not connected to the dynamical event of core bounce, it lasts for a prolonged interval, and can eventually radiate more energy than the dynamical event itself. The characteristic frequencies of the gravitational radiation from the overturning motions range from $225 \mathrm{~Hz}$ to $960 \mathrm{~Hz}$.

\section{EVOLUTION OF THE ROTATION RATE}

The evolution of the rotation parameter $\beta$ and the angular velocity is of particular interest, since they are connected to still unanswered questions in core-collapse supernovae physics: What is the period of newborn neutron stars? Can protoneutron stars become dynamically or secularily unstable to triaxial rotational modes?

Our study is Newtonian and lacks a full treatment of the microphysics involved. Even though we have included realistic presupernova models and a realistic equation of state, we cannot claim to provide final answers to the above questions. Moreover, our calculations have been done in 2D, not 3D, and, thus, are not free of symmetry constraints. General relativistic gravity would lead to more compact configurations with higher $\beta \mathrm{s}$. Hence, the results presented in this section should be seen only as indications of the systematic behavior of the rotation rate evolution and of the changes in the distribution of angular velocity with total angular momentum.
A look at Fig. 7 and at Tables 3, 4, and 5 discloses that there exists a maximum value of $\beta$ at bounce for a given progenitor model and value of A. Interestingly, the maximum $\beta$ is not reached by the model with the maximum $\beta_{i}$, but by a model with some intermediate value of $\beta_{i}$ (in Fig. 7 this is $0.40 \%$ ). $\beta$ at bounce is determined by the subtle interplay between initial angular momentum distribution, the equation of state, centrifugal forces and gravity. The "optimal" configuration leads to the overall maximum $\beta$ at bounce for a given $\beta_{i}$. Moreover, the model yielding the maximum final $\beta$ is not necessarily the model yielding the maximum $\beta$ at bounce (see Fig. 7 and Tables 3-6).

Figure 19 shows the final angular velocity distribution versus radius at the equator of the $\mathrm{s} 15$ model for $\mathrm{A}=500 \mathrm{~km}$ and $\mathrm{A}=50000 \mathrm{~km}$ and for a variety of $\beta_{i} \mathrm{~s}$. As with $\beta$, overall the angular velocity increases with increasing $\beta_{i}$ until a maximum is reached. It subsequently decreases with the further increase of $\beta_{i}$. Both initial settings of A lead to strongly differential rotation in the central regions, while the initially more rigidly rotating model ( $A=50000 \mathrm{~km}$, solid lines in Fig. 19) actually yields larger post-bounce angular velocity gradients inside 30 $\mathrm{km}$. Its equatorial velocity profile peaks off center for moderate $\beta_{i}$ at radii between 6 and $8 \mathrm{~km}$. The initially more differentially rotating model $(A=500 \mathrm{~km}$, dashed lines in Fig. 19) leads to the highest central values of the angular velocity while its angular velocity profile quickly drops to low values and practically rigid rotation for $\beta_{i} \geq 0.3 \%$. Model s15A500 $\beta 0.2$ results in the shortest rotation period near the center $(\sim 1.5 \mathrm{~ms})$. Model s15A50000 $\beta 0.5$ yields the shortest period of the $\mathrm{A}=50000 \mathrm{~km}$ model series $(\sim 1.85 \mathrm{~ms})$. The angular velocity shear exterior to the peak at 6-8 $\mathrm{km}$ exhibited by these models has also been identified in the one-dimensional study of Akiyama et al. (2003). These authors consider such shear a possible driver for the magneto-rotational instability (MRI), which could be a generator of strong magnetic fields.

None of our models develop off-center density maxima (i.e. become toroidal) which could lead to dynamical growth of an azimuthal $\mathrm{m}=1$ mode at $\beta \gtrsim 14 \%$ as suggested by Centrella et al. (2001). Shibata, Karino, and Eriguchi (2003) have shown that strongly differentially rotating polytropes with centrally peaked density profiles and EOS- $\Gamma$ above $\sim 4 / 3$ can become dynamically unstable to triaxial $\mathrm{m}=2$ deformations (bar modes) for $\beta$ on the order of 1\%. As seen in Fig. 19 and discussed in the previous paragraph, our models exhibit strong differential rotation inside the protoneutron star. However, it is impossible to judge whether they are stable or unstable to triaxial rotational instabilities in context of Shibata, Karino, and Eriguchi (2003), since these protoneutron stars differ greatly from the simple polytropic equilibrium models employed in that study. Three-dimensional simulations employing a finite-temperature EOS and realistic post-collapse models are needed to address this issue conclusively.

\section{PROSPECTS FOR DETECTION}

To assess the detectability of the gravitational waves radiated by our models we follow the discussions in Abramovici et al. (1992) and Flanagan and Hughes (1998). For a given frequency, $f$, Flanagan and Hughes (1998) define the characteristic gravitational wave strain 


$$
h_{\text {char }}(f)=\sqrt{\frac{2}{\pi^{2}} \frac{G}{c^{3}} \frac{1}{D^{2}} \frac{d E(f)}{d f},}
$$

where $\mathrm{D}$ is the distance of the source from the detector (for galactic sources we set this equal to $10 \mathrm{kpc})$ and $d E(f) / d f$ is the spectral energy density of the gravitational radiation defined by eq. (22).

The matched-filter signal-to-noise ratio for a source that emits at optimal orientation and polarization is given by

$$
\left(S N R_{\text {optimal }}\right)^{2}=\int d(\ln f) \frac{h_{\text {char }}(f)^{2}}{h_{r m s}(f)^{2}}
$$

with the (single) detector rms noise strain $h_{r m s}$ being defined as the square root of frequency times the detector noise power spectral density

$$
h_{r m s}(f)=\sqrt{f S(f)} .
$$

For two observatories, averaging over all angles and both polarizations, and assuming a SNR of 5, the detector burst sensitivity is considered to be $h_{S B} \simeq 11 h_{r m s}$ (Abramovici et al. 1992; Gustafson et al. 1999).

Figure 20 shows the detector $h_{r m s}$ noise strains of the initial and advanced LIGO designs (Gustafson et al. 1999). The solid squares mark the peaks of the optimal characteristic strain $h_{\text {char }}\left(f_{\max }\right)$ for each of our models at an assumed distance of 10 $\mathrm{kpc}$. The most important parameters governing the position of a given model in Fig. 20 are $\beta_{i}$ and A. Generally, the models with moderate initial rotation $\left(\beta_{i}\right.$ below $\left.0.5 \%\right)$ peak at frequencies well above the LIGO peak sensitivity (near $100 \mathrm{~Hz}$ ), but also give the largest overall gravitational wave amplitudes. With increasing $\beta_{i}$, the models shift towards lower frequencies and smaller amplitudes, but at $D=10 \mathrm{kpc}$ remain for the most part above the initial LIGO sensitivity limit. Only for the strongest rotators, which peak at low frequencies, will detectability at 10 $\mathrm{kpc}$ by the initial LIGO interferometers be problematic. For a given $\beta_{i}$, the model spectra shift upwards and to the right with increasing A. Overall, our s20 models peak at the largest $h_{\text {char }}$. The peaks of the s11 and s15 models are very similar for slow rotation, but the fast rotating s11 models peak at lower frequencies and higher strains than the corresponding s15 models. If we were to draw an imaginary line through the field of points on Fig. 20, we would find that peak $h_{\text {char }}$ is very roughly proportional to $f_{\max }$ to the 0.8 power.

The models evolved without rotation are marked with little stars in Fig. 20. Models marked with a diamond correspond to rotating progenitors from the studies of Heger, Langer, and Woosley (2000) and Heger et al. (2003). The three models at higher frequencies below the sensitivity of the first-generation (initial) LIGO are the three "magnetic" progenitors that rotate very slowly $\left(\beta_{i}<0.01 \%\right)$. In sum, approximately $80 \%$ of our models are within the optimal sensitivity limit of the firstgeneration LIGO and about $10 \%$ should be detectable even using the $h_{S B}$ condition. Almost all of our models should be detectable with the 2nd-generation (advanced) LIGO.

\section{SUMMARY AND DISCUSSION}

In this parameter study, we have investigated the emission of gravitational radiation from rotational stellar core collapse using Newtonian gravity, realistic initial models, and a realistic equation of state. We have performed a total of 72 simulations, investigating the dependence upon the degree of differential rotation, the initial ratio of rotational energy to gravitational potential energy $\left(\beta_{i}\right)$, and progenitor. For this, we employed the 11,15 and $20 \mathrm{M}_{\odot}$ (s11, s15, s20) presupernova models from the stellar evolution study of Woosley and Weaver (1995) and put them into rotation via a rotation law that assumes rotation on cylinders. In addition, we have performed simulations with the progenitor models of Heger, Langer, and Woosley (2000) and Heger et al. (2003) that include a one-dimensional prescription for rotational evolution. All of our models encompass the full $180^{\circ}$ of the symmetry domain. Nothing artificial was done to initiate collapse.

Our results indicate that there are two types of characteristic behavior for the collapse dynamics and the resulting gravitational wave signature. Type I occurs for slow initial rotation and is characterized by core bounce at supranuclear densities. The wave signal of a type I model exhibits a sharp spike and high frequency oscillations as the compact remnant rings down after core bounce. Type I models have the largest gravitational wave amplitudes, their energy spectra peak at the highest frequencies and they radiate the largest amount of energy. On the other hand, type II models bounce at subnuclear densities due to the influence of centrifugal forces. They exhibit several damped harmonic oscillator-like post-bounce expansioncollapse-bounce cycles whose periods grow with increasing $\beta_{i}$. The gravitational wave amplitudes, frequencies, and total energies of type II models are smaller than for those that exhibit type I behavior. The frequency at which the coherent postbounce oscillations occur is clearly discernible in the energy spectra of type II models. Figure 21 portrays the gravitational wave energy spectrum for the s11A500 sequence at various $\beta_{i}$ s from $0.1 \%$ to $0.5 \%$. This figure is similar to Fig. 10 for the s151000 series. The systematic shift with increasing $\beta_{i}$ above the transition $\beta_{i}(\sim 0.25 \%)$ from higher frequencies and strength to lower values of both is clearly seen. Transitional models that exhibit features of both type I and type II behavior are also present. For a given initial model, we find that the transition from type I to type II happens for smaller $\beta_{i}$ if the core is initially more differentially rotating and for larger $\beta_{i}$ if its core has rigid initial rotation. The s11 and s15 progenitor models give very similar results, the s11 model giving slightly larger gravitational wave amplitudes, frequencies, and energies than the s15 model for a given set of parameters. The s20 model, however, having a shallower initial density distribution, leads to different post-bounce behavior, since its hydrodynamic bounce shock stalls. Subsequent to stall, matter infalls aspherically through and onto the central core. This leads to near-critical damping of the post-bounce oscillations of the compact remnant and introduces high-frequency components into the wave form. Since sophisticated one-dimensional studies that include weak interaction physics and neutrino transport indicate that the bounce shock does indeed initially stall (Rampp and Janka 2002; Liebendörfer et al. 2001a,b; Thompson, Burrows, and Pinto 2003), the distinctive character of the s20 model may in fact be more generic than that of our s11 and s15 models.

The rotating progenitor models e 15 and e 20 from Heger, Langer, and Woosley (2000) behave similarly to their s15 and s20 counterparts with similar $\beta_{i}$ s and As. However, due to differences in presupernova structure, the bounce shock of the e 15 model stalls, leading to more infall into the central core and 
strong damping of post-bounce oscillations. The same is true for the e20 model. Hence, the gravitational wave signatures for both models are similar to those for the s 20 models. The models of Heger et al. (2003) include the effects of magnetic fields on the distribution of angular momentum. This leads to very rigid rotation throughout the star and, thus, to little angular momentum in the iron core itself. Our simulations show that these extremely slowly rotating cores $\left(\beta_{i}<0.005 \%\right)$ yield gravitational wave amplitudes from core bounce that are two orders of magnitude smaller than for the s11, s15 and s20 models with the smallest $\beta_{i}$. Hence, the quantitative and qualitative behavior of these slowly rotating models is very similar to the models that were evolved without any rotation at all.

Our models yield absolute values of the dimensionless maximum gravitational wave strain in the interval $2.0 \times 10^{-23} \leq$ $h_{\max }^{T T} \leq 1.25 \times 10^{-20}$ at a distance of $10 \mathrm{kpc}$. The total energy radiated $\left(E_{G W}\right)$ lies in the range $1.4 \times 10^{-11} \mathrm{M}_{\odot} \mathrm{c}^{2} \leq E_{G W} \leq$ $2.21 \times 10^{-8} \mathrm{M}_{\odot} \mathrm{c}^{2}$ and the energy spectra peak (with the exception of very few models) in the frequency interval $20 \mathrm{~Hz}$ $\lesssim \mathrm{f}_{\text {peak }} \lesssim 600 \mathrm{~Hz}$. Furthermore, our hydrodynamic results indicate (if the canonical critical conditions apply), that none of our collapsed cores will undergo triaxial rotational instabilities, since the maximum ratio of rotational to gravitational energy $(\beta)$ reached is $9.16 \%$ at bounce and $8.23 \%$ at the end of the evolution. Both these values are below the putative critical $\beta$ for dynamical $\left(\beta_{d y n} \simeq 27 \%\right)$ or secular $\left(\beta_{\text {sec }} \simeq 14 \%\right)$ triaxial instability (in the simplified case of a MacLaurin spheroid). However, given the extreme density profiles in realistic progenitor cores, and the canonical bifurcation of the flow into outer supersonic and inner subsonic collapse, the actual critical $\beta$ s for triaxial deformation of realistic cores have yet to be determined. Recent studies by Centrella et al. (2001) and Shibata, Karino, and Eriguchi (2003) which employed polytropic neutron star models suggest that dynamical rotational instabilities could occur at much lower $\beta$. These results have to be verified in three-dimensional simulations including a finite-temperature EOS and realistic protoneutron star models.

Since including weak-interaction physics and neutrino transport will change the overall evolution, our specific results for the $\beta_{i}$ dependence should be taken with care. However, the qualitative behavior and trends we have identified should be robust.

In comparison with the studies of Zwerger and Müller (1997) (Newtonian gravity) and Dimmelmeier, Font, and Müller (2002b) (relativistic gravity), who used polytropic progenitors and a simplified equation of state, our models radiate on average about an order of magnitude less energy, exhibit a factor of 4 to 10 smaller maximum wave amplitudes, and peak at lower frequencies. Zwerger and Müller (1997) categorized the collapse, bounce and post-bounce behavior of their models in three types of which their types I and II match our types I and II. However, we have not encountered their type III behavior ("rapid collapse") in any of our calculations.

Aside from this study, there have been only two major efforts to calculate the gravitational wave signature of core collapse with a sophisticated realistic equation of state and realistic progenitor models. These were Mönchmeyer et al. (1991) and the recent study by Kotake, Yamada, and Sato (2003). Both studies included electron capture and a leakage scheme for approximate treatment of neutrino transport, but calculated only a very small set of models and used only one pre-supernova progenitor. In accord with our results, none of them found $\mathrm{Zw}$ erger and Müller (1997) type III behavior. Their gravitational wave amplitudes and total radiated energies are qualitatively and quantitatively similar to ours.

To assess the detectability of gravitational waves from core collapse, we applied the method proposed by Flanagan and Hughes (1998). We find that at a distance of $10 \mathrm{kpc}$, i.e. for galactic distances, the 1st-generation LIGO, once it has reached its design sensitivity level, will be able to detect more than $80 \%$ of our core collapse models under optimal conditions and orientations. Assuming random polarizations and angles of incidence, this reduces to $10 \%$. Advanced LIGO, however, should be able to detect virtually all models at galactic distances. Figure 22 is similar to Fig. 20 in that it presents peak $h_{\text {char }}$, but it also includes the actual $h_{\text {char }}$ spectra of selected models (eq. 25). These spectra are complementary to the energy spectra of previous figures, and serve to put the issues of detectability in a noisy detector into sharper relief.

To conclude, we point out that even though this study has advanced our knowledge of gravitational waves from core collapse a bit further, many important questions remain unanswered. Detailed microphysics and neutrino transport are apt to change the dynamics of collapse, bounce and post-bounce phases. The coherent post-bounce oscillations of type II models will most likely be strongly damped by the stalled bounce shock in simulations that account for neutrino energy losses. Furthermore, gravitational radiation is to be expected from anisotropic neutrino emission (Burrows and Hayes 1996), and neutrinodriven convection behind the stalled shock and in the protoneutron star. Both sources are potentially significant emitters of gravitational wave energy.

The detection of gravitational radiation from collapse supernovae would open a new window into the violent dynamics at the core of the supernova and neutron-star birth phenomena. Furthermore, features seen in gravitational radiation may have their counterparts in the neutrino signal. Seeing a correlation in these two disparate channels could break the study of supernovae wide open. As we have shown, measurements of wave frequency, wave form, and power can in principle reveal the rotational structure of the massive star interior that, though of central importance across a broad spectrum of astrophysics, has to date remained almost completely out of reach.

We acknowledge helpful conversations with Todd Thompson, Itamar Lichtenstadt, Harald Dimmelmeier, Ian Hawke, Chris Fryer, Ed Seidel, Sam Finn, Joan Centrella, Kim New, Wolfgang Duschl, Casey Meakin, Jeremiah Murphy and Sven Marnach. In addition, we thank Jeff Fookson and Neal Lauver of the Steward Computer Support Group for their invaluable help with the local Beowulf cluster. Support for this work is provided in part by the Scientific Discovery through Advanced Computing (SciDAC) program of the DOE, grant number DEFC02-01ER41184. 


\section{REFERENCES}

Abramovici, A. et al. 1992, Science, 256, 325

Akiyama, S. et al. 2003, ApJ, 584, 954

Ando, M. et al. 2001, Phys. Rev. Lett., 86, 3950

Akiyama, S., Wheeler, J.C., Meier, D., and Lichtenstadt, I. 2003, ApJ, 584, 954

Bethe, H. A. and Wilson, J. R. 1985, ApJ, 295, 14

Blanchet, L., Damour, T., and Schäfer, G. 1990, MNRAS, 242, 289

Bonazzola, S. and Marck, J. A. 1993, A\&A, 267, 623

Brown, J. D. 2001, in AIP Conf. Proc. 575: Astrophysical Sources for GroundBased Gravitational Wave Detectors, 234

Buras, R., Rampp, M., Janka, H.-T., and Kifonidis, K. 2003, astro-ph/0303171

Burrows, A. and Hayes, J. 1996, Phys. Rev. Lett, 76, 352

Burrows, A., Hayes, J., and Fryxell, B. A. 1995, ApJ, 450, 830

Burrows, A. and Thompson, T. A. 2003, in From Twilight to Highlight: The Physics of Supernovae. Proceedings of the ESO/MPA/MPE Workshop held in Garching, Germany, 29-31 July 2002, 53

Centrella, J., New, K., Lowe, L., and Brown, J. 2001, ApJ, 550, L193

Dimmelmeier, H., Font, J., and Müller, E. 2002a, A\&A, 388, 917

-. 2002b, A\&A, 393, 523

Epstein, R. 1978, ApJ, 223, 1037

Eriguchi, Y. and Müller, E. 1985, A\&A, 146, 260

Evans, C. R. 1986, in Dynamical Spacetimes and Numerical Relativity, ed. J. M. Centrella (Cambridge, U. K.: Cambridge University Press), 3-39

Finn, L. S. and Evans, C. R. 1990, ApJ, 351, 588

É. É. Flanagan and Hughes, S. A. 1998, Phys. Rev. D, 57, 4535

Fryer, C., Benz, W., Herant, M., and Colgate, S. A. 1999, ApJ, 516, 892

Fryer, C., Holz, D., and Hughes, S. 2002, ApJ, 565, 430

Fryer, C. L. and Heger, A. 2000, ApJ, 541, 1033

Fukuda, I. 1982, PASP, 94, 271

Gustafson, E., Shoemaker, D., Strain, K., and Weiss, R. 1999, LSC white paper on detector research and development, Technical Report LIGO T990080-00-D

Hachisu, I. 1986a, ApJS, 61, 479

-. 1986b, ApJS, 62, 461

Heger, A., Fryer, C. L., Woosley, S. E., Langer, N., and Hartmann, D. H. 2002, astro-ph/0212469

Heger, A., Langer, N., and Woosley, S. E. 2000, ApJ, 528, 368

Heger, A., Woosley, S. E., Langer, N., and Spruit, H. C. 2003, Stellar Rotation. Proceedings IAU Symposium No. 215

Hillebrandt, W. and Wolff, R. G. 1985, in Nucleosynthesis: Challenges and New Developments, eds. D. Arnett and J. W. Truran (Chicago, U.S.A.: University Chicago Press)

Ipser, J. R. and Managan, R. A. 1984, ApJ, 282, 287

Janka, H.-T., Zwerger, T., and Mönchmeyer, R. 1993, A\&A, 268, 360

Kotake, K., Yamada, S., and Sato, K. 2003, Phys. Rev. D, in press

Lattimer, J. M., Pethick, C., Ravenhall, D., and Lamb, D. 1985, Nucl. Phys. A, 432, 646

Lattimer, J. M. and Swesty, F. D. 1991, Nucl. Phys. A, 535, 331

Ledoux, P. 1945, ApJ, 102, 143

Liebendörfer, M., Mezzacappa, A., and Thielemann, F. 2001a, Phys. Rev. D, 63, 104003

Liebendörfer, M., Mezzacappa, A., Thielemann, F., Messer, O. E., Hix, W. R., and Bruenn, S. W. 2001b, Phys. Rev. D, 63, 103004
Livne, E. 1993, ApJ, 412, 634

Misner, C. W., Thorne, K. S., and Wheeler, J. A. 1973, Gravitation (San Francisco, U. S. A.: Freeman)

Mönchmeyer, R., Schäfer, G., Müller, E., and Kates, R. 1991, A\&A, 246, 417

Moncrief, V. 1979, ApJ, 234, 628

Müller, E. 1982, A\&A, 114, 53

Müller, E. and Hillebrandt, W. 1981, A\&A, 103, 358

Müller, E. and Janka, H.-T. 1997, A\&A, 317, 140

Nakamura, T. and Oohara, K. 1989, in Frontiers in Numerical Relativity, ed. C. R. Evans, L. S. Finn, and D. W. Hobill (Cambridge, U. K.: Cambridge University Press), 254

New, K. C. B. 2003, Living Rev. Relativity 6, 2. [Online Article]: cited on 20. July 2003, http://www.livingreviews.org/lrr-2003-2

Punturo, M., VIRGO Note, The VIRGO sensitivity curve, VIR-NOT-PER-1390-51

Press, W. H., Teukolsky, S. A., Vetterling, W. T., and Flannery, B. P. 1992, Numerical Recipes in C, 2nd. edition (Cambridge, U. K.: Cambridge University Press)

Rampp, M. and Janka, H.-T. 2000, ApJ, 539, L33

-. 2002, A\&A, 396, 361

Rampp, M., Müller, E., and Ruffert, M. 1998, A\&A, 332, 969

Saenz, R. A. and Shapiro, S. L. 1978, ApJ, 221, 286

-. 1979, ApJ, 229, 1107

-. 1981, ApJ, 244, 1033

Seidel, E. and Moore, T. 1987, Phys. Rev. D, 35, 2287

Seidel, E., Myra, E. S., and Moore, T. 1988, Phys. Rev. D, 38, 2349

Shibata, M., Karino, S., Eriguchi, Y. 2002, MNRAS, 334, L27

Shibata, M., Karino, S., Eriguchi, Y. 2003, MNRAS, 343, 619

Shapiro, L. S. and Teukolsky, S. A. 1983, Black Holes, White Dwarfs and Neutron Stars (New York U. S. A.: John Wiley \& Sons)

Shapiro, S. L. and Lightman, A. P. 1976, ApJ, 207, 263

Shapiro, S. L. 1977, ApJ, 214, 566

Shen, H., Toki, H., Oyamatsu, K., and Sumiyoshi, K. 1998, Nucl. Phys. A, 637, 43

Tassoul, J.-L. 1978, Theory of Rotating Stars (Princeton U. S. A.: Princeton University Press)

Thompson, T. A., Burrows, A., and Pinto, P. A. 2003, ApJ 592, July 20, in press (astro-ph/0211194)

Thorne, K. S. 1980, Reviews of Modern Physics, 52, 299

Timmes, F. X. and Arnett, D. 1999, ApJS, 125, 277

Timmes, F. X. and Swesty, F. D. 2000, ApJS, 126, 501

Tohline, J. E. 1984, ApJ, 361, 394

Turner, M. S. and Wagoner, R. V. 1979, in Sources of Gravitational Radiation, ed. L. Smarr (Cambridge, U. K.: Cambridge University Press)

Weaver, T. A., Zimmerman, G. B., and Woosley, S. E. 1978, ApJ, 225, 102

Willke, B. et al. 2002, Classical and Quantum Gravity, 19, 1377

Woosley, S. E., Heger, A., and Weaver, T. A. 2002, Reviews of Modern Physics, 74,1015

Woosley, S. E. and Weaver, T. A. 1995, ApJS, 101, 181

-. 1995, ApJ, 450, 245

Zwerger, T. and Müller, E. 1997, A\&A, 320, 209 
TABLE 1

INITIAL MODEL DATA

\begin{tabular}{lrrcccc}
\hline \hline Model Name & $\begin{array}{c}R_{c} \\
\left(10^{8} \mathrm{~cm}\right)\end{array}$ & $\begin{array}{c}M_{c} \\
\left(\mathrm{M}_{\odot}\right)\end{array}$ & $\begin{array}{c}\text { ZAMS Mass } \\
\left(\mathrm{M}_{\odot}\right)\end{array}$ & $\begin{array}{c}\text { ZAMS } v_{e q} \\
\left(\mathrm{~km} \mathrm{~s}^{-1}\right)\end{array}$ & $\begin{array}{c}\beta_{i} \\
(\%)\end{array}$ & Reference \\
\hline n=3 polytrope & 1.55 & 1.46 & & & & Zwerger and Müller (1997) \\
s11WW & 1.33 & 1.31 & 11 & & & Woosley and Weaver (1995) \\
s15WW & 1.16 & 1.28 & 15 & & & Woosley and Weaver (1995) \\
s20WW & 2.21 & 1.73 & 20 & & & Woosley and Weaver (1995) \\
s25WW & 2.28 & 1.78 & 25 & & & Woosley and Weaver (1995) \\
e15 & 1.21 & 1.33 & 15 & 200 & 0.650 & Heger, Langer, and Woosley (2000) \\
e20 & 2.19 & 1.68 & 20 & 200 & 0.420 & Heger, Langer, and Woosley (2000) \\
m15b4 & 1.23 & 1.37 & 15 & 200 & 0.002 & Heger et al. (2003) \\
m20b4 & 1.53 & 1.49 & 20 & 200 & 0.003 & Heger et al. (2003) \\
m25b4 & 1.93 & 1.64 & 25 & 200 & 0.005 & Heger et al. (2003) \\
\hline
\end{tabular}

Note. - List of progenitor models used in this paper. $R_{c}$ is the radius of the iron core (determined by the discontinuity in $Y_{e}$ at the outer edge of the iron core), $M_{c}$ is the mass of the core, $v_{e q}$ the equatorial velocity of the model at ZAMS, and $\beta_{i}$ is the initial rotation parameter. All progenitor models already have an initial infall velocity profile when they are mapped onto our 2-dimensional grid. Nothing artificial is done to initiate collapse.

TABLE 2

RESUlTS: POLYTROPES

\begin{tabular}{lccccccccccc}
\hline \hline Model & $\begin{array}{c}\beta_{i} \\
(\%)\end{array}$ & $\begin{array}{c}\mathrm{J} \\
\left(10^{49}\right. \\
\mathrm{erg} \mathrm{s})\end{array}$ & $\begin{array}{c}\mathrm{t}_{b} \\
(\mathrm{~ms})\end{array}$ & $\begin{array}{c}\Delta \mathrm{t} \\
(\mathrm{ms})\end{array}$ & $\begin{array}{c}\beta_{b} \\
(\%)\end{array}$ & $\begin{array}{c}\rho_{b} \\
\left(10^{14}\right. \\
\left.\mathrm{g} \mathrm{cm}^{-3}\right)\end{array}$ & $\begin{array}{c}A_{20 \max }^{E 2} \\
(\mathrm{~cm})\end{array}$ & $\begin{array}{r}h_{\max }^{T T} \\
\left(10^{-21}\right)\end{array}$ & $\begin{array}{r}f_{\max } \\
(\mathrm{Hz})\end{array}$ & $\begin{array}{c}E_{G W, b} \\
\left(10^{-9}\right. \\
\left.\mathrm{M}_{\odot} \mathrm{c}^{2}\right)\end{array}$ & $\begin{array}{c}E_{G W, f} \\
\left(10^{-9}\right. \\
\left.\mathrm{M}_{\odot} \mathrm{c}^{2}\right)\end{array}$ \\
\hline A50000 $\beta 0.25$ & 0.25 & 1.273 & 66.78 & 83.22 & 4.03 & 3.52 & -876.19 & -7.76 & 540 & 14.50 & 18.28 \\
A50000 $\beta 0.50$ & 0.50 & 1.746 & 67.51 & 82.49 & 6.75 & 3.26 & -1617.13 & -14.31 & 453 & 41.43 & 56.31 \\
A50000 $\beta 0.90$ & 0.90 & 2.370 & 68.90 & 81.11 & 9.97 & 2.83 & -2125.30 & -18.81 & 460 & 49.24 & 57.08 \\
A1000 $\beta 0.25$ & 0.25 & 1.227 & 67.05 & 82.95 & 5.28 & 3.43 & -1211.62 & -10.72 & 467 & 25.85 & 36.04 \\
A1000 $\beta 0.50$ & 0.50 & 1.646 & 67.97 & 82.03 & 8.40 & 3.10 & -2151.21 & -19.04 & 693 & 65.61 & 71.57 \\
A1000 $\beta 0.90$ & 0.90 & 2.314 & 70.11 & 79.89 & 12.48 & 2.36 & -1674.60 & -14.82 & 313 & 17.71 & 19.58 \\
A1000 $\beta 1.80$ & 1.80 & 3.281 & 74.34 & 46.26 & 13.35 & 0.40 & -800.78 & -7.09 & 100 & 14.35 & 1.60 \\
A500 $\beta 0.25$ & 0.25 & 1.130 & 67.43 & 79.57 & 6.80 & 3.30 & -1782.64 & -15.78 & 509 & 50.22 & 64.22 \\
A500 $\beta 0.90$ & 0.90 & 2.177 & 71.81 & 78.19 & 13.58 & 1.19 & -1317.80 & -11.66 & 173 & 7.41 & 8.08 \\
NONROT & - & - & 65.96 & 84.04 & - & 3.78 & -14.82 & -0.01 & 500 & - & 0.01 \\
\hline
\end{tabular}

Note. - Overview of core collapse simulations performed with the polytropic progenitor model and the hybrid equation of state (§4.1) for comparison with Zwerger and Müller (1997) and Dimmelmeier, Font, and Müller (2002b). $\beta_{i}$ is the initial rotation parameter, $\mathbf{J}$ is the total angular momentum, and $\mathrm{t}_{b}, \beta_{b}$ and $\rho_{b}$ are the time, central density, and rotation parameter at core bounce. $\Delta \mathrm{t}=\mathrm{t}_{f}-\mathrm{t}_{b}$ is the time each calculation was carried out beyond core bounce. $\left|A_{20}^{E 2}\right|_{\max }$ and $h_{\max }$ are the absolute maximum of the gravitational quadrupole wave amplitude and the maximum gravitational wave strain, as defined in $\S 4.3 . f_{\max }$ is the peak frequency of the gravitational wave spectrum, $E_{G W, b}$ is the gravitational wave energy radiated up to the first post-bounce minimum in the maximum density, and $E_{G W, f}$ is the total energy radiated in gravitational waves during the entire simulation. 
TABLE 3

Results: Realistic Progenitor Model s11WW

\begin{tabular}{|c|c|c|c|c|c|c|c|c|c|c|c|}
\hline Model & $\begin{array}{c}\beta_{i} \\
(\%)\end{array}$ & $\begin{array}{c}\mathbf{J} \\
\left(10^{49}\right. \\
\text { erg s })\end{array}$ & $\begin{array}{c}\Delta \mathrm{t} \\
(\mathrm{ms})\end{array}$ & $\begin{array}{c}\beta_{b} \\
(\%)\end{array}$ & $\begin{array}{c}\beta_{f} \\
(\%)\end{array}$ & $\begin{array}{c}\rho_{b} \\
\left(10^{14}\right. \\
\left.\mathrm{g} \mathrm{cm}^{-3}\right) \\
\end{array}$ & $\begin{array}{c}A_{20 \max }^{E 2} \\
(\mathrm{~cm})\end{array}$ & $\begin{array}{c}h_{\max }^{T T} \\
@ 10 \mathrm{kpc} \\
\left(10^{-21}\right)\end{array}$ & $\begin{array}{l}f_{\max } \\
(\mathrm{Hz})\end{array}$ & $\begin{array}{c}E_{G W, b} \\
\left(10^{-9}\right. \\
\left.\mathrm{M}_{\odot} \mathrm{c}^{2}\right)\end{array}$ & $\begin{array}{c}E_{G W, f} \\
\left(10^{-9}\right. \\
\left.\mathrm{M}_{\odot} \mathrm{c}^{2}\right)\end{array}$ \\
\hline s11A500 $\beta 0.1$ & 0.10 & 0.708 & 167 & 4.00 & 3.62 & 3.70 & -958.99 & -8.49 & 524 & 10.99 & 11.88 \\
\hline $\mathrm{s} 11 \mathrm{~A} 500 \beta 0.2$ & 0.20 & 1.002 & 145 & 7.05 & 5.86 & 3.30 & -1408.17 & -12.46 & 402 & 19.15 & 22.11 \\
\hline $\mathrm{s} 11 \mathrm{~A} 500 \beta 0.25$ & 0.25 & 1.097 & 135 & 7.87 & 6.43 & 3.10 & -1203.06 & -10.65 & 404 & 11.21 & 12.78 \\
\hline s11A $500 \beta 0.3$ & 0.30 & 1.227 & 413 & 8.80 & 5.19 & 2.74 & -661.27 & -5.85 & 164 & 1.70 & 1.81 \\
\hline s11A $500 \beta 0.4$ & 0.40 & 1.417 & 468 & 8.00 & 4.81 & 1.11 & -419.65 & -3.71 & 37 & 0.46 & 0.55 \\
\hline $\mathrm{s} 11 \mathrm{~A} 500 \beta 0.5$ & 0.50 & 1.584 & 707 & 7.71 & 4.80 & 0.46 & -325.27 & -2.88 & 25 & 0.18 & 0.22 \\
\hline $\mathrm{s} 11 \mathrm{~A} 1000 \beta 0.1$ & 0.10 & 0.803 & 179 & 3.33 & 3.03 & 3.73 & -878.86 & -7.78 & 430 & 8.96 & 10.04 \\
\hline $\mathrm{s} 11 \mathrm{~A} 1000 \beta 0.2$ & 0.20 & 1.135 & 182 & 6.06 & 4.03 & 3.41 & -1304.82 & -11.55 & 470 & 14.10 & 15.53 \\
\hline s11A $1000 \beta 0.3$ & 0.30 & 1.391 & 91 & 8.00 & 6.21 & 3.00 & -861.14 & -7.62 & 208 & 3.82 & 4.26 \\
\hline s11A $1000 \beta 0.4$ & 0.40 & 1.606 & 304 & 7.98 & 4.77 & 2.06 & -378.58 & -3.35 & 150 & 0.35 & 0.42 \\
\hline s11A $1000 \beta 0.5$ & 0.50 & 1.795 & 284 & 7.40 & 4.65 & 0.60 & -301.88 & -2.66 & 78 & 0.16 & 0.19 \\
\hline s11A $1000 \beta 0.6$ & 0.60 & 1.967 & 503 & 7.15 & 4.49 & 0.28 & -229.35 & -2.02 & 38 & 0.06 & 0.07 \\
\hline s11A $1000 \beta 0.7$ & 0.70 & 2.124 & 424 & 6.95 & 4.41 & 0.15 & -183.83 & -1.63 & 26 & 0.03 & 0.03 \\
\hline s11A $1000 \beta 0.8$ & 0.80 & 2.271 & 510 & 6.66 & 4.38 & 0.09 & -139.37 & -1.23 & 44 & 0.01 & 0.02 \\
\hline $\mathrm{s} 11 \mathrm{~A} 50000 \beta 0.1$ & 0.10 & 0.856 & 172 & 2.17 & 1.97 & 3.83 & -582.09 & -5.15 & 394 & 3.93 & 4.86 \\
\hline $\mathrm{s} 11 \mathrm{~A} 50000 \beta 0.2$ & 0.20 & 1.211 & 156 & 3.88 & 3.32 & 3.64 & -979.41 & -8.67 & 416 & 9.03 & 9.72 \\
\hline s11A50000 $\beta 0.25$ & 0.25 & 1.354 & 69 & 4.73 & 3.83 & 3.55 & -1111.64 & -9.84 & 409 & 10.00 & 10.57 \\
\hline $\mathrm{s} 11 \mathrm{~A} 50000 \beta 0.3$ & 0.30 & 1.483 & 229 & 5.47 & 4.51 & 3.45 & -1188.38 & -10.52 & 420 & 10.11 & 10.61 \\
\hline s11A50000 $\beta 0.4$ & 0.40 & 1.713 & 163 & 6.47 & 5.42 & 3.23 & -1075.10 & -9.52 & 344 & 6.20 & 6.48 \\
\hline $\mathrm{s} 11 \mathrm{~A} 50000 \beta 0.5$ & 0.50 & 1.914 & 90 & 7.38 & 4.11 & 2.97 & -613.39 & -5.43 & 166 & 1.36 & 1.52 \\
\hline s11A50000 $\beta 0.6$ & 0.60 & 2.097 & 249 & 7.34 & 4.30 & 2.37 & -266.17 & -2.36 & 99 & 0.16 & 0.19 \\
\hline $\mathrm{s} 11 \mathrm{~A} 50000 \beta 0.7$ & 0.70 & 2.266 & 404 & 6.78 & 4.36 & 0.86 & -238.21 & -2.11 & 54 & 0.08 & 0.10 \\
\hline s11nonrot & - & - & 88 & - & - & 4.04 & -26.09 & -0.02 & 275 & - & 0.06 \\
\hline
\end{tabular}

Note. - Numerical results of core collapse simulations performed with the Woosley and Weaver (1995) s11 progenitor model in conjunction with the Lattimer-Swesty equation of state (Lattimer and Swesty 1991; §4.1). $\beta_{i}$ is the initial rotation parameter, J is the total angular momentum, and $\beta_{b}$ and $\rho_{b}$ are the central density and rotation parameter at core bounce. $\Delta \mathrm{t}=\mathrm{t}_{f}-\mathrm{t}_{b}$ is the time each individual calculation was carried out beyond core bounce. $\beta_{f}$ is the final rotation parameter for the whole grid. $\left|A_{20}^{E 2}\right|_{\max }$ and $h_{\max }$ are the absolute maximum of the gravitational quadrupole wave amplitude and the maximum gravitational wave strain, as defined in §4.3. $f_{\max }$ is the peak frequency of the gravitational wave spectrum, $E_{G W . b}$ gives the gravitational wave energy radiated before the first post-bounce minimum in the maximum density, and $E_{G W, f}$ is the total energy radiated in gravitational waves during the entire simulation. 
TABLE 4

Results: Realistic Progenitor Model s15WW

\begin{tabular}{|c|c|c|c|c|c|c|c|c|c|c|c|}
\hline Model & $\begin{array}{c}\beta_{i} \\
(\%)\end{array}$ & $\begin{array}{c}\mathbf{J} \\
\left(10^{49}\right. \\
\text { erg s })\end{array}$ & $\begin{array}{c}\Delta \mathrm{t} \\
(\mathrm{ms})\end{array}$ & $\begin{array}{c}\beta_{b} \\
(\%)\end{array}$ & $\begin{array}{c}\beta_{f} \\
(\%)\end{array}$ & $\begin{array}{c}\rho_{b} \\
\left(10^{14}\right. \\
\left.\mathrm{g} \mathrm{cm}^{-3}\right)\end{array}$ & $\begin{array}{c}A_{20 \max }^{E 2} \\
(\mathrm{~cm})\end{array}$ & $\begin{array}{c}h_{\max }^{T T} \\
@ 10 \mathrm{kpc} \\
\left(10^{-21}\right)\end{array}$ & $\begin{array}{l}f_{\max } \\
(\mathrm{Hz})\end{array}$ & $\begin{array}{c}E_{G W, b} \\
\left(10^{-9}\right. \\
\left.\mathrm{M}_{\odot} \mathrm{c}^{2}\right)\end{array}$ & $\begin{array}{c}E_{G W, f} \\
\left(10^{-9}\right. \\
\left.\mathrm{M}_{\odot} \mathrm{c}^{2}\right)\end{array}$ \\
\hline $\mathrm{s} 15 \mathrm{~A} 500 \beta 0.1$ & 0.10 & 0.811 & 215 & 3.87 & 3.60 & 3.69 & -862.97 & -7.64 & 524 & 9.06 & 10.14 \\
\hline $\mathrm{s} 15 \mathrm{~A} 500 \beta 0.2$ & 0.20 & 1.147 & 181 & 6.88 & 5.79 & 3.31 & -1283.00 & -11.36 & 469 & 16.76 & 17.88 \\
\hline s $15 \mathrm{~A} 500 \beta 0.25$ & 0.25 & 1.257 & 151 & 7.83 & 6.55 & 3.12 & -1205.50 & -10.67 & 381 & 11.87 & 13.32 \\
\hline $\mathrm{s} 15 \mathrm{~A} 500 \beta 0.3$ & 0.30 & 1.409 & 138 & 8.71 & 5.99 & 2.80 & -758.40 & -6.71 & 242 & 2.69 & 2.88 \\
\hline s15A500 $\beta 0.4$ & 0.40 & 1.557 & 251 & 8.10 & 5.35 & 1.31 & -426.44 & -3.77 & 169 & 0.51 & 0.52 \\
\hline $\mathrm{s} 15 \mathrm{~A} 500 \beta 0.5$ & 0.50 & 1.812 & 641 & 7.81 & 5.19 & 0.54 & -343.78 & -3.04 & 128 & 0.23 & 0.25 \\
\hline s15A500 $\beta 0.6$ & 0.60 & 1.987 & 373 & 7.71 & 5.10 & 0.29 & -278.79 & -2.47 & 101 & 0.11 & 0.12 \\
\hline $\mathrm{s} 15 \mathrm{~A} 500 \beta 0.9$ & 0.90 & 2.421 & 524 & 7.52 & 5.05 & 0.07 & -171.30 & -1.52 & 55 & 0.02 & 0.03 \\
\hline $\mathrm{s} 15 \mathrm{~A} 500 \beta 1.0$ & 1.00 & 2.564 & 661 & 7.40 & 5.03 & 0.05 & -138.20 & -1.22 & 42 & 0.01 & 0.01 \\
\hline $\mathrm{s} 15 \mathrm{~A} 1000 \beta 0.1$ & 0.10 & 0.958 & 90 & 3.15 & 2.63 & 3.75 & -742.56 & -6.57 & 431 & 6.56 & 7.90 \\
\hline $\mathrm{s} 15 \mathrm{~A} 1000 \beta 0.2$ & 0.20 & 1.355 & 178 & 5.43 & 4.82 & 3.49 & -1164.47 & -10.31 & 461 & 12.32 & 13.10 \\
\hline $\mathrm{s} 15 \mathrm{~A} 1000 \beta 0.3$ & 0.30 & 1.660 & 167 & 7.11 & 6.37 & 3.13 & -1040.73 & -9.21 & 317 & 7.10 & 7.58 \\
\hline s15A1000 $\beta 0.4$ & 0.40 & 1.916 & 312 & 8.37 & 5.48 & 2.66 & -491.54 & -4.35 & 152 & 0.83 & 0.89 \\
\hline s $15 \mathrm{~A} 1000 \beta 0.5$ & 0.50 & 2.142 & 208 & 7.67 & 5.22 & 1.08 & -362.96 & -3.21 & 106 & 0.27 & 0.29 \\
\hline s15A1000 $\beta 0.6$ & 0.60 & 2.292 & 251 & 7.43 & 5.03 & 0.49 & -293.17 & -2.59 & 91 & 0.13 & 0.13 \\
\hline s15A1000 $\beta 0.7$ & 0.70 & 2.535 & 313 & 7.29 & 4.80 & 0.26 & -234.48 & -2.08 & 64 & 0.06 & 0.07 \\
\hline $\mathrm{s} 15 \mathrm{~A} 1000 \beta 0.8$ & 0.80 & 2.710 & 284 & 7.19 & 4.77 & 0.16 & -190.31 & -1.68 & 38 & 0.03 & 0.03 \\
\hline s15A1000 $\beta 0.9$ & 0.90 & 2.633 & 437 & 7.11 & 4.74 & 0.10 & -152.94 & -1.35 & 31 & 0.02 & 0.02 \\
\hline $\mathrm{s} 15 \mathrm{~A} 1000 \beta 1.0$ & 0.80 & 2.906 & 441 & 6.97 & 4.72 & 0.06 & -124.39 & -1.10 & 22 & 0.01 & 0.01 \\
\hline $\mathrm{s} 15 \mathrm{~A} 50000 \beta 0.1$ & 0.10 & 1.081 & 207 & 1.40 & 1.41 & 3.90 & -382.21 & -3.38 & 396 & 1.65 & 2.16 \\
\hline $\mathrm{s} 15 \mathrm{~A} 50000 \beta 0.2$ & 0.20 & 1.528 & 108 & 2.79 & 2.28 & 3.77 & -654.29 & -5.79 & 400 & 4.90 & 5.95 \\
\hline $\mathrm{s} 15 \mathrm{~A} 50000 \beta 0.5$ & 0.50 & 2.416 & 243 & 5.49 & 4.94 & 3.41 & -1034.23 & -9.15 & 428 & 7.46 & 7.48 \\
\hline $\mathrm{s} 15 \mathrm{~A} 50000 \beta 1.0$ & 1.00 & 3.417 & 494 & 7.42 & 4.65 & 2.16 & -299.88 & -2.65 & 71 & 0.18 & 0.20 \\
\hline s15nonrot & - & - & 60 & - & - & 4.04 & -23.87 & -0.02 & 337 & - & 0.02 \\
\hline
\end{tabular}

Note. - Same as Table 3 but for all simulations using the Woosley and Weaver (1995) s15 progenitor model in conjunction with the Lattimer-Swesty equation of state. 
TABLE 5

Results: Realistic Progenitor Model s20WW/S25WW

\begin{tabular}{|c|c|c|c|c|c|c|c|c|c|c|c|}
\hline Model & $\begin{array}{l}\beta_{i} \\
(\%)\end{array}$ & $\begin{array}{c}\mathbf{J} \\
\left(10^{49}\right. \\
\text { erg s })\end{array}$ & $\begin{array}{c}\Delta \mathrm{t} \\
(\mathrm{ms})\end{array}$ & $\begin{array}{l}\beta_{b} \\
(\%)\end{array}$ & $\begin{array}{l}\beta_{f} \\
(\%)\end{array}$ & $\begin{array}{c}\rho_{b} \\
\left(10^{14}\right. \\
\left.\mathrm{g} \mathrm{cm}^{-3}\right)\end{array}$ & $\begin{array}{l}A_{20 \max }^{E 2} \\
\quad(\mathrm{~cm})\end{array}$ & $\begin{array}{c}h_{\max }^{T T} \\
@ 10 \mathrm{kpc} \\
\left(10^{-21}\right)\end{array}$ & $\begin{array}{l}f_{\max } \\
(\mathrm{Hz})\end{array}$ & $\begin{array}{c}E_{G W, b} \\
\left(10^{-9}\right. \\
\left.\mathbf{M}_{\odot} \mathrm{c}^{2}\right)\end{array}$ & $\begin{array}{c}E_{G W, f} \\
\left(10^{-9}\right. \\
\left.\mathbf{M}_{\odot} \mathrm{c}^{2}\right)\end{array}$ \\
\hline s20A500 $\beta 0.1$ & 0.10 & 1.326 & 103 & 5.50 & 4.97 & 3.45 & -1139.52 & -10.09 & 594 & 16.03 & 19.03 \\
\hline $\mathrm{s} 20 \mathrm{~A} 500 \beta 0.2$ & 0.20 & 1.882 & 142 & 8.96 & 7.64 & 2.78 & -1077.88 & -9.54 & 396 & 6.55 & 8.85 \\
\hline $\mathrm{s} 20 \mathrm{~A} 500 \beta 0.5$ & 0.50 & 2.976 & 416 & 8.65 & 8.00 & 0.22 & -355.85 & -3.15 & 92 & 0.16 & 0.22 \\
\hline $\mathrm{s} 25 \mathrm{~A} 500 \beta 0.2$ & 0.20 & 2.217 & 56 & 9.16 & 7.55 & 2.72 & -1043.73 & -9.24 & 453 & 5.90 & 8.07 \\
\hline s20A $1000 \beta 0.1$ & 0.10 & 1.941 & 52 & 4.51 & 4.01 & 3.61 & -954.61 & -8.45 & 525 & 10.90 & 12.67 \\
\hline $\mathrm{s} 20 \mathrm{~A} 1000 \beta 0.2$ & 0.20 & 2.745 & 94 & 7.71 & 7.00 & 3.14 & -1247.85 & -11.04 & 387 & 14.90 & 18.76 \\
\hline $\mathrm{s} 20 \mathrm{~A} 1000 \beta 0.3$ & 0.30 & 3.304 & 146 & 9.12 & 7.52 & 2.44 & -678.39 & -6.00 & 73 & 1.43 & 1.74 \\
\hline s20A $1000 \beta 0.4$ & 0.40 & 2.858 & 189 & 8.49 & 7.71 & 0.82 & -536.00 & -4.74 & 85 & 0.66 & 0.78 \\
\hline $\mathrm{s} 20 \mathrm{~A} 1000 \beta 0.5$ & 0.50 & 3.984 & 113 & 8.37 & 7.45 & 0.36 & -424.96 & -3.76 & 97 & 0.30 & 0.33 \\
\hline s20A $1000 \beta 0.6$ & 0.60 & 4.254 & 291 & 8.37 & 8.10 & 0.20 & -345.78 & -3.06 & 79 & 0.15 & 0.17 \\
\hline s20A $1000 \beta 0.7$ & 0.70 & 4.549 & 290 & 8.31 & 8.17 & 0.12 & -269.03 & -2.38 & 66 & 0.07 & 0.08 \\
\hline $\mathrm{s} 20 \mathrm{~A} 1000 \beta 0.8$ & 0.80 & 4.839 & 300 & 8.33 & 8.23 & 0.08 & -220.16 & -1.95 & 56 & 0.02 & 0.03 \\
\hline s20A $1000 \beta 0.9$ & 0.90 & 5.040 & 282 & 8.32 & 8.15 & 0.05 & -185.81 & -1.64 & 47 & 0.02 & 0.03 \\
\hline $\mathrm{s} 20 \mathrm{~A} 1000 \beta 1.0$ & 1.00 & 5.226 & 316 & 8.36 & 8.17 & 0.04 & -154.43 & -1.37 & 16 & 0.01 & 0.01 \\
\hline s20A50000 $\beta 0.1$ & 0.10 & 1.950 & 40 & 1.53 & 1.26 & 3.89 & -351.07 & -3.11 & 409 & 1.25 & 1.79 \\
\hline s $20 \mathrm{~A} 50000 \beta 0.2$ & 0.20 & 2.758 & 36 & 2.89 & 2.34 & 3.78 & -619.26 & -5.48 & 424 & 4.48 & 5.49 \\
\hline s20A50000 $\beta 0.5$ & 0.50 & 4.361 & 230 & 5.88 & 5.96 & 3.39 & -1175.30 & -10.40 & 456 & 10.14 & 11.06 \\
\hline s20A50000 $\beta 1.0$ & 1.00 & 6.168 & 303 & 8.38 & 7.02 & 2.36 & -449.58 & -3.98 & 102 & 0.61 & 0.68 \\
\hline s20nonrot & - & - & 83 & - & - & 4.08 & -165.02 & -1.46 & 472 & - & 1.07 \\
\hline
\end{tabular}

Note. - Same as Table 3, but for all simulations using the Woosley and Weaver (1995) s20 and s25 progenitor models. The s25 run justifies our assumption in $\$ 2$ that the s20 and s 25 progenitors lead to similar results. Note that for the s 25 run a larger grid was used and the evolution was stopped before the post-bounce oscillations had faded. Hence, the differences seen in the total angular momentum and maximum frequency.

TABLE 6

Results: Heger Models

\begin{tabular}{lccccccccccc}
\hline \hline Model & $\begin{array}{c}\beta_{i} \\
(\%)\end{array}$ & $\begin{array}{c}\mathrm{J} \\
\left(10^{49}\right. \\
\mathrm{erg} \mathrm{s})\end{array}$ & $\begin{array}{c}\Delta \mathrm{t} \\
(\mathrm{ms})\end{array}$ & $\begin{array}{c}\beta_{b} \\
(\%)\end{array}$ & $\begin{array}{c}\beta_{f} \\
(\%)\end{array}$ & $\begin{array}{c}\rho_{b} \\
\left(10^{14}\right. \\
\left.\mathrm{g} \mathrm{cm}^{-3}\right)\end{array}$ & $\begin{array}{c}A_{20 \max }^{E 2} \\
(\mathrm{~cm})\end{array}$ & $\begin{array}{c}h_{\max }^{T T} \\
\text { @ } 10 \mathrm{kpc} \\
\left(10^{-21}\right)\end{array}$ & $\begin{array}{c}f_{\max } \\
(\mathrm{Hz})\end{array}$ & $\begin{array}{c}E_{G W, b} \\
\left(10^{-9}\right. \\
\left.\mathrm{M}_{\odot} \mathrm{c}^{2}\right)\end{array}$ & $\begin{array}{c}E_{G W, f} \\
\left(10^{-9}\right. \\
\left.\mathrm{M}_{\odot} \mathrm{c}^{2}\right)\end{array}$ \\
\hline m15b4 & 0.0021 & 0.1345 & 30 & 0.087 & 0.082 & 4.14 & -29.15 & -0.26 & 590 & 0.004 & 0.014 \\
m20b4 & 0.0032 & 0.3034 & 37 & 0.161 & 0.156 & 4.15 & -52.46 & -0.46 & 360 & 0.026 & 0.041 \\
m25b4 & 0.0053 & 0.3561 & 94 & 0.324 & 0.360 & 4.14 & -101.06 & -0.89 & 960 & 0.110 & 0.550 \\
\hline e15 & 0.6454 & 3.392 & 311 & 8.82 & 7.49 & 0.27 & -405.57 & -3.59 & 90 & 0.235 & 0.275 \\
e20 & 0.4176 & 3.442 & 294 & 8.52 & 7.87 & 0.55 & -436.77 & -3.87 & 193 & 0.465 & 0.631 \\
\hline
\end{tabular}

Note. - Same as Table 3, but for all simulations using the Heger, Langer, and Woosley (2000) and Heger et al. (2003) progenitor models with rotation. Model $\mathrm{m} 25 \mathrm{~b} 4$, evolved long enough for convective instability behind the shock to grow, shows considerable energy radiated by the post-bounce convective bulk mass motions. 


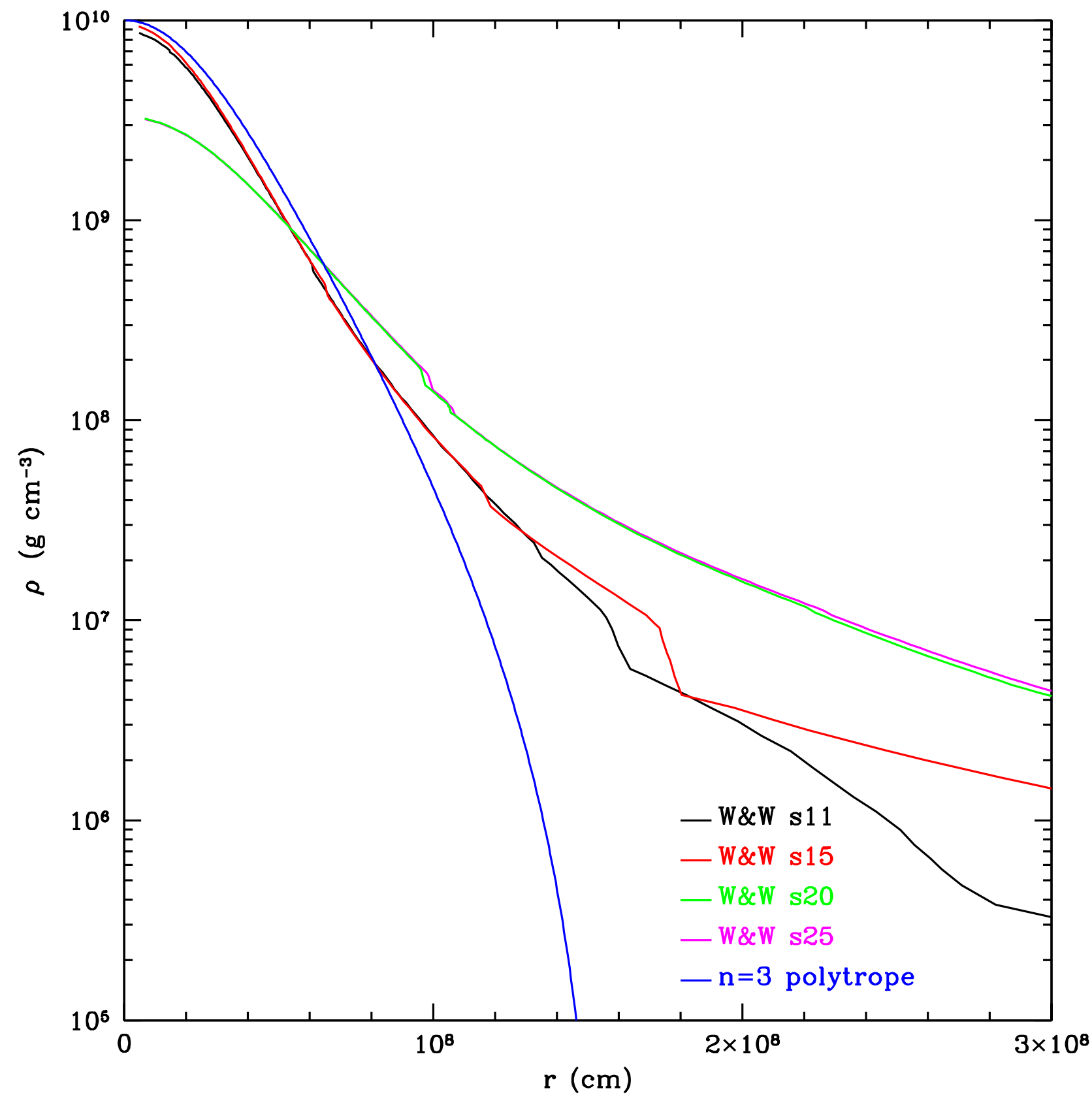

FIG. 1.- Mass density as a function of radius of the Woosley and Weaver (1995) progenitor models and the $n=3$ polytropic progenitor of Zwerger and Müller (1997). Note the good correspondence in the central regions between the less massive iron cores and the polytrope, but the general disagreement at larger radii. The polytrope has a sharper edge. 


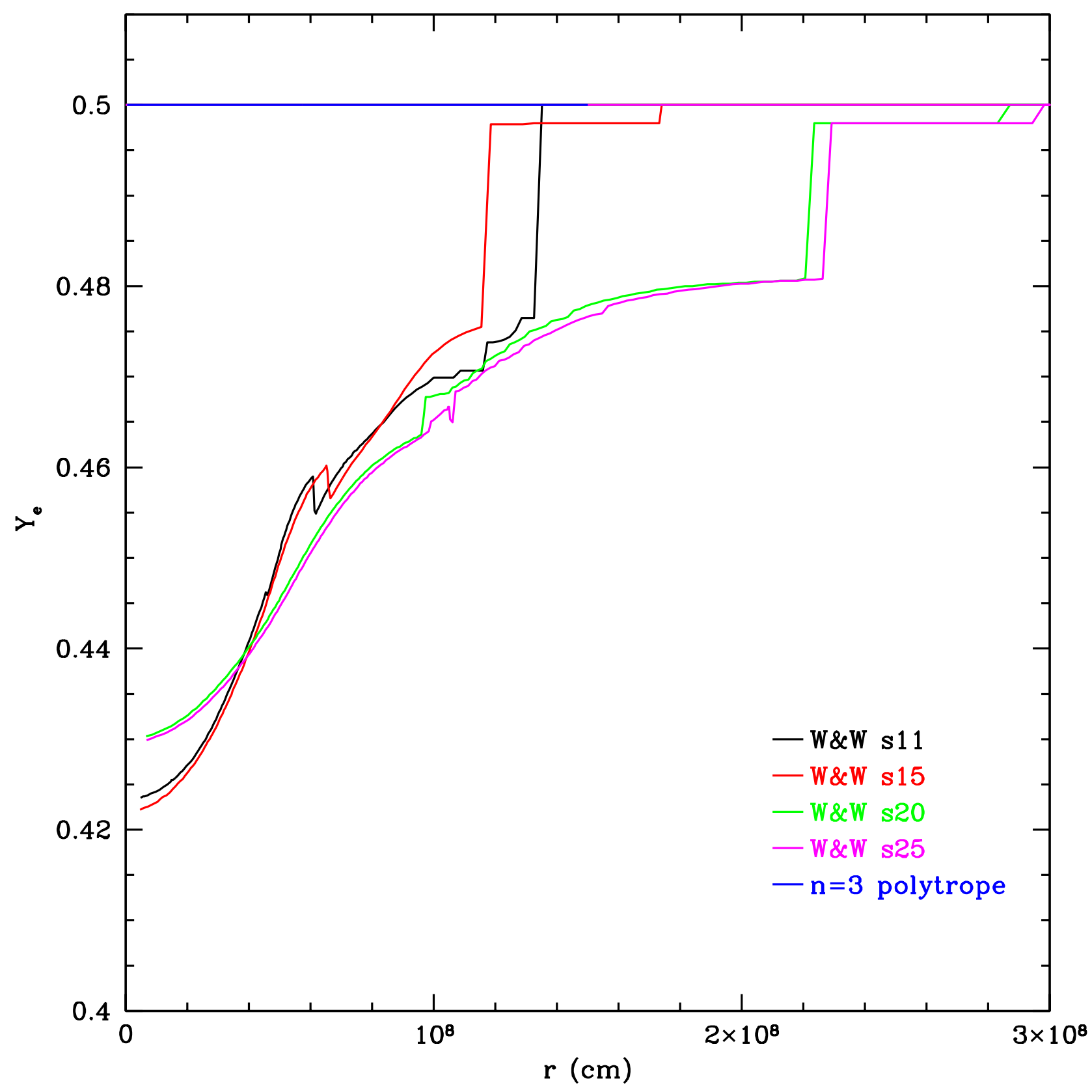

FIG. 2.- Electron number fraction $Y_{e}$ as a function of radius for the Woosley and Weaver (1995) progenitor models. The central iron core has been neutronized due to electron capture during core silicon burning (Weaver, Zimmerman, and Woosley 1978). For the polytrope, $Y_{e}$ has been assumed to be 0.5 (Zwerger and Müller 1997). The discontinuity in $Y_{e}$ exhibited in the Woosley and Weaver models indicates the approximate boundary between the central iron core and the surrounding shells. 


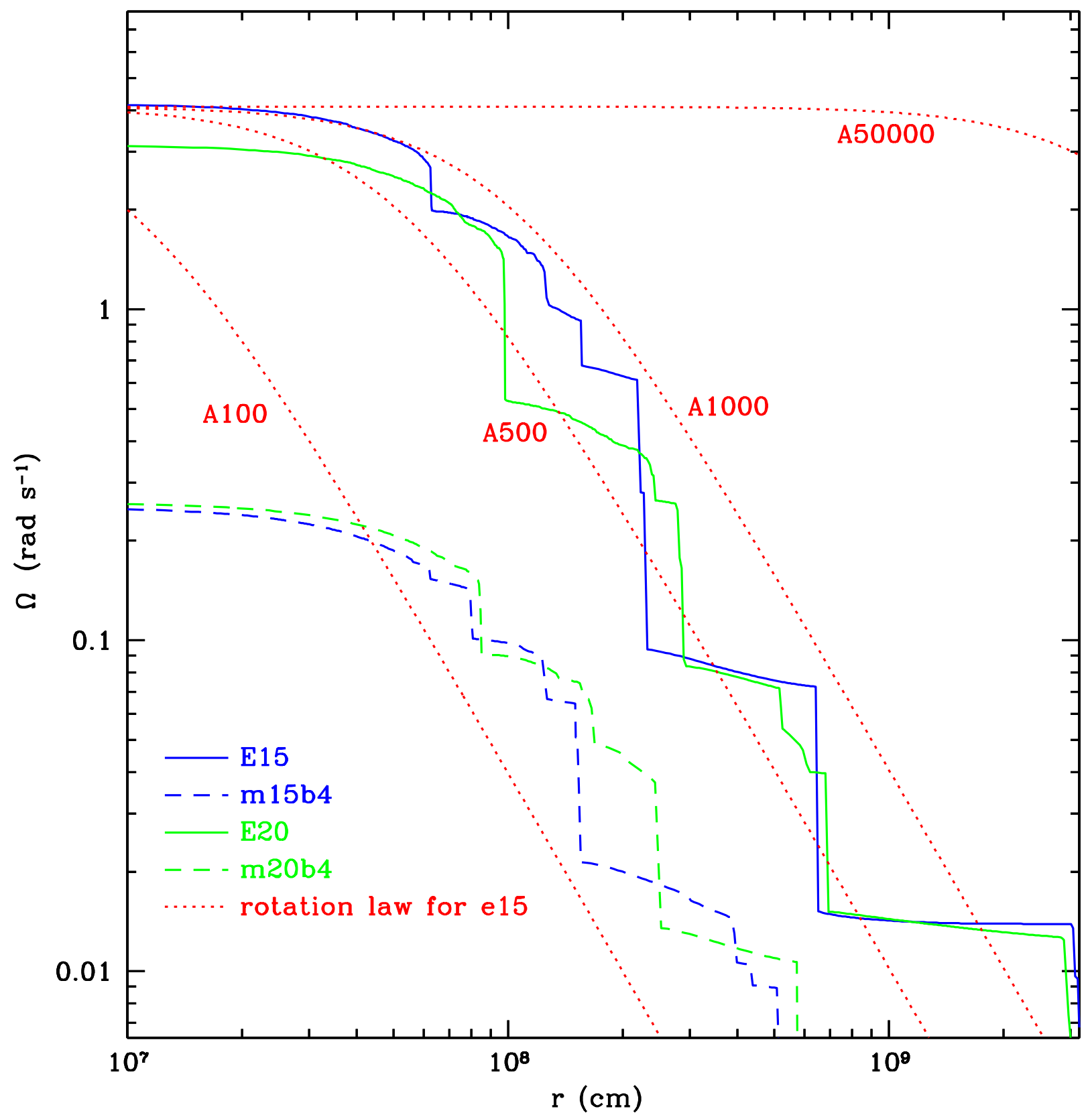

FIG. 3.- Initial angular velocity profiles of the rotating 15 (blue) and 20 (green) $\mathrm{M}_{\odot}$ progenitor models (see Table 1 for model parameters). The dotted red profiles were generated with the rotation law of eq. (5) using the central $\Omega$ of model e15 for $\Omega_{0}$. All realistic presupernova models exhibit near rigid rotation inside $\simeq 1000 \mathrm{~km}$. Note the much smaller angular velocities exhibited by models m15b4 and m20b4, which were evolved with the inclusion of magnetic fields. 


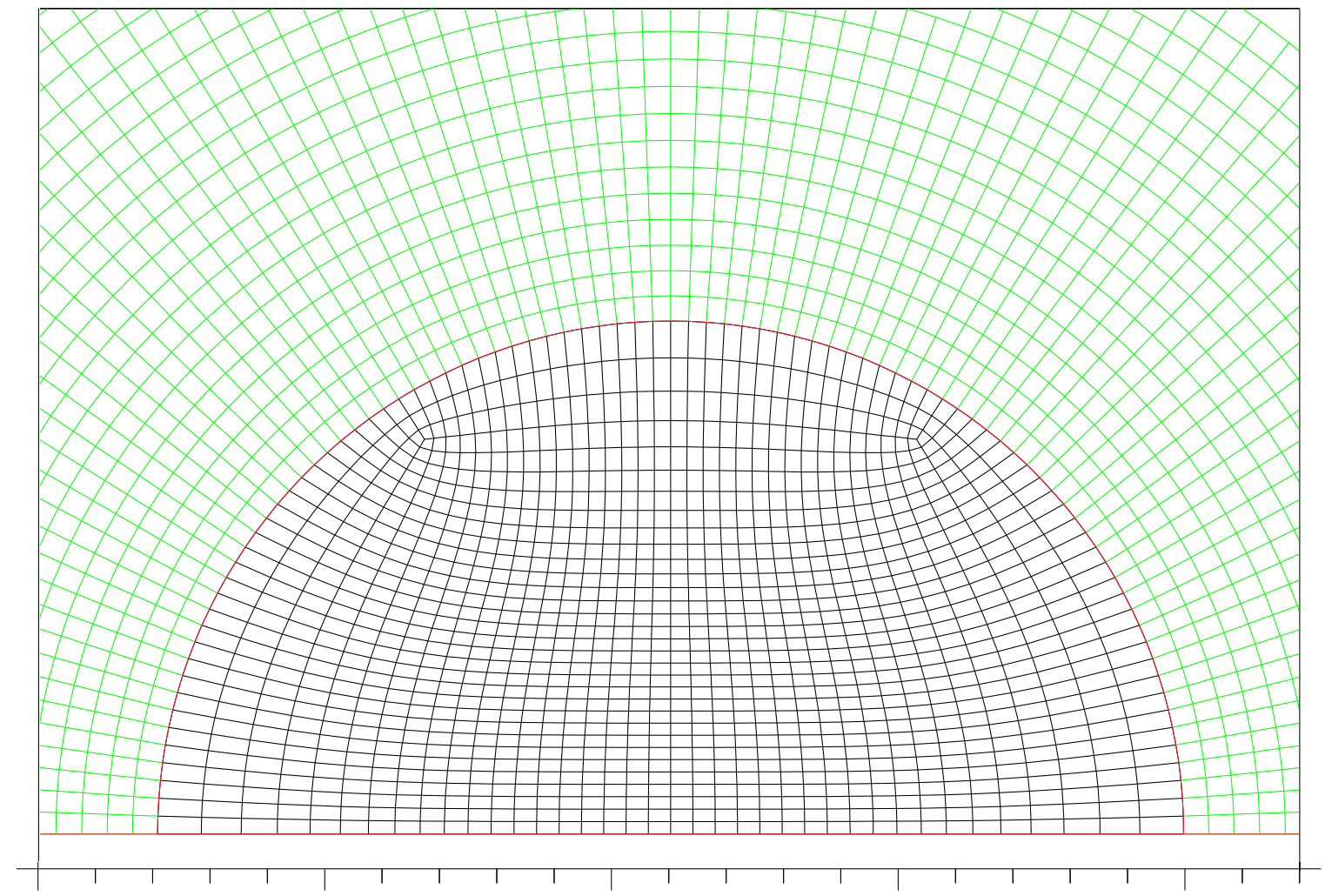

FIG. 4.-Central region of the VULCAN/2D computational grid. Shown in black is the inner $10 \mathrm{~km}$, where the capability of VULCAN/2D to work with arbitrary grid configurations has been used to perform a smooth transition from the outer radial grid to a Cartesian grid in the innermost region. 

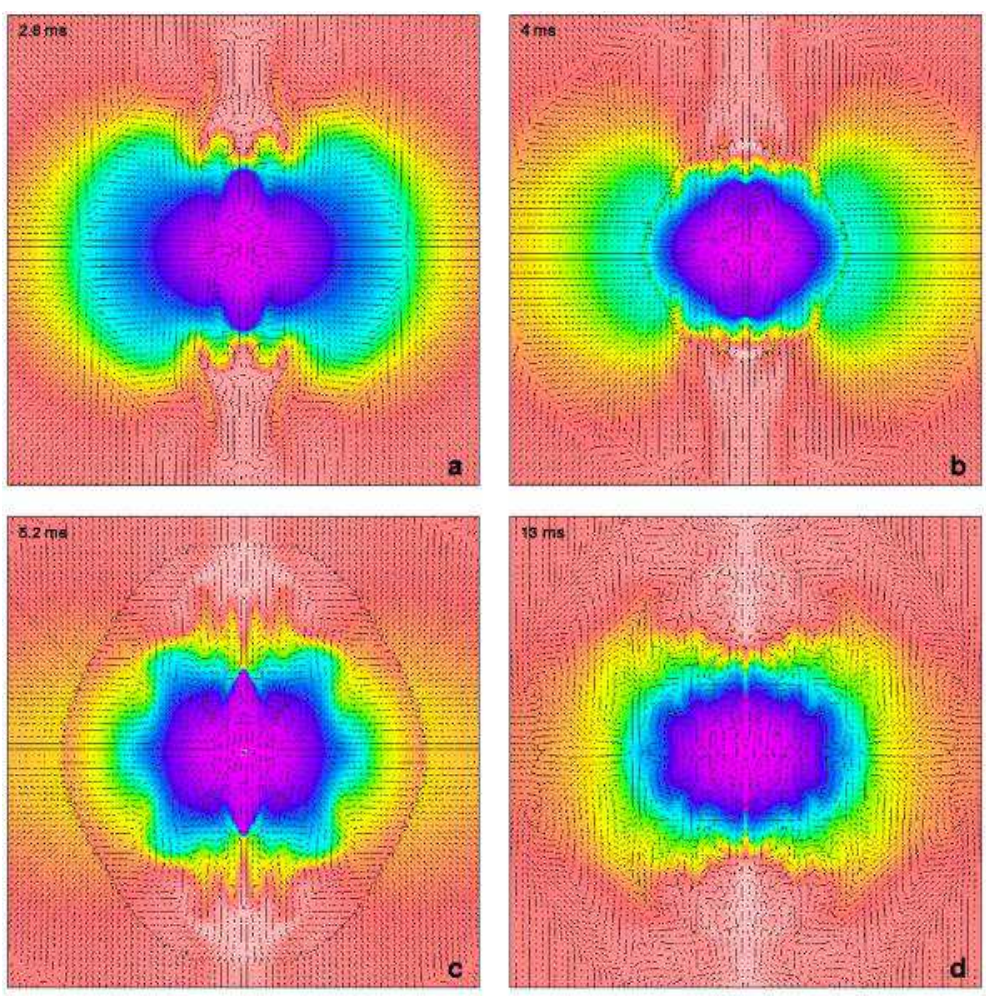

FIG. 5.- 2-D plots of the specific entropy of the inner $200 \times 200 \mathrm{~km}^{2}$ of the $\mathrm{s} 15 \mathrm{~A} 1000 \beta 0.2$ model depicting the ring-down epoch of the evolution of this generic type I model. The color map goes from light red for high specific entropy $\left(s=16 \mathrm{k}_{B}\right)$ to magenta for low entropy $\left(s=0.7 \mathrm{k}_{B}\right)$. The times given in the top left-hand corners are the snapshot times after bounce. Velocity vectors for the $r$ and $\theta$ motions (not the $\phi$ motion in the angular direction) are superposed. As the core executes successive radial and non-radial post-bounce oscillations, it generates strong sound waves (weak shock waves) that are seen in panels (b) and (c) as contours of velocity discontinuity. At the time of panel (a), the actual bounce shock has already left the frame. Panel (d) shows the compact remnant at 13 ms after bounce when it has already lost most of its excess pulsational energy. The velocities at that time are dominated by incoherent vortical motions. (This figure is available in high-quality format from http://www.ita.uni-heidelberg.de/ ${ }^{\circ}$ cott/gwpaper .) 


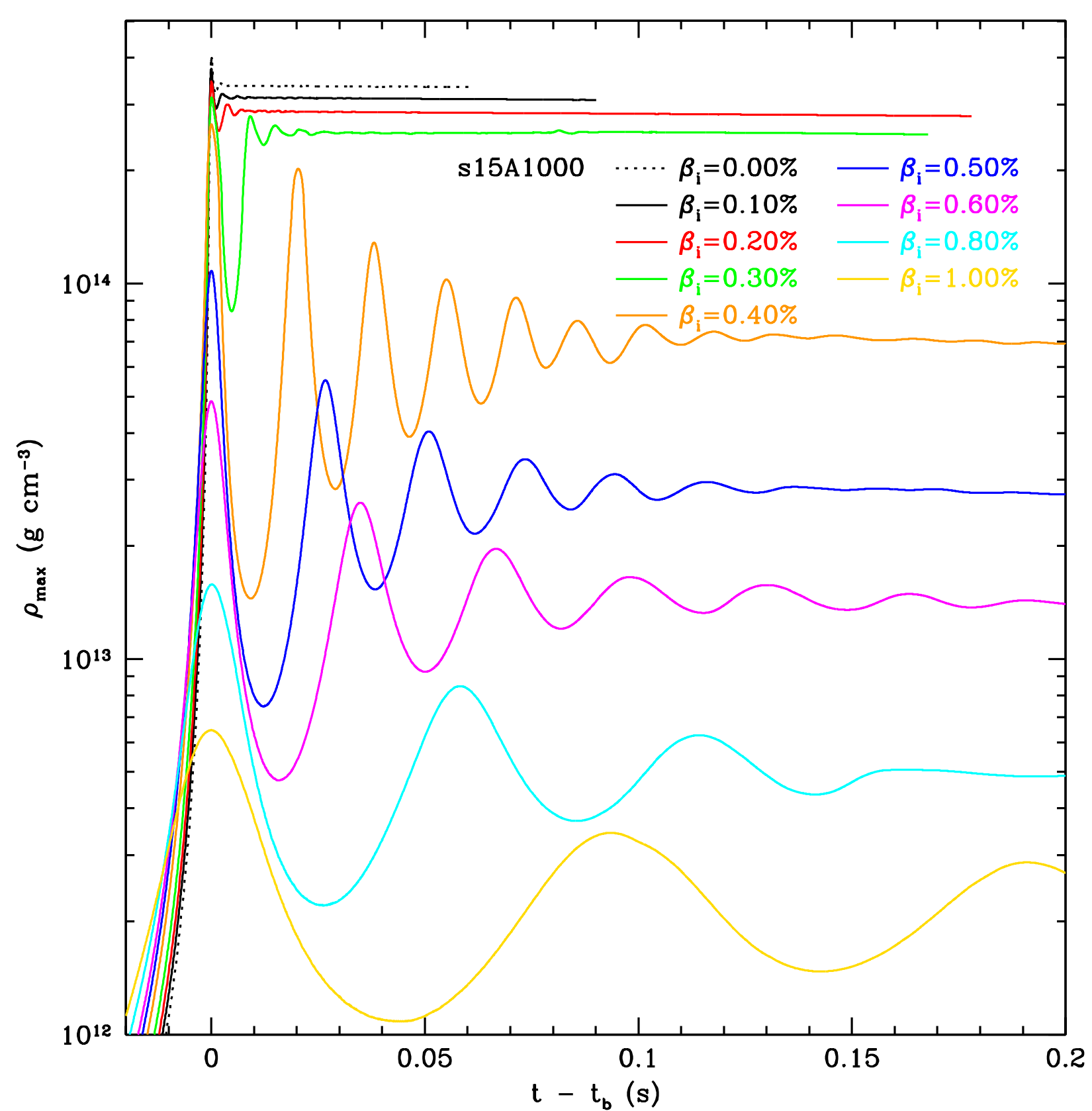

FIG. 6. - Evolution of the maximum density of the s15A1000 model series, which rotates almost rigidly inside $1000 \mathrm{~km}$, for different initial rotation parameters $\beta_{i}$. For comparision, the dashed line shows the evolution of the central density of a nonrotating model. The models on this graph undergo core bounce between 280 and $500 \mathrm{~ms}$ after the start of the evolution. The time to bounce increases with initial rotation rate since centrifugal forces, acting as additional pressure support, slow down collapse. All times are relative to the time of bounce $\left(t_{b}\right)$ for each individual model. One can clearly see the transition in collapse dynamics that takes place in the interval in $\beta$-space between $0.20 \%$ and $0.40 \%$ which leads from type I to type II behavior (see text for details). 


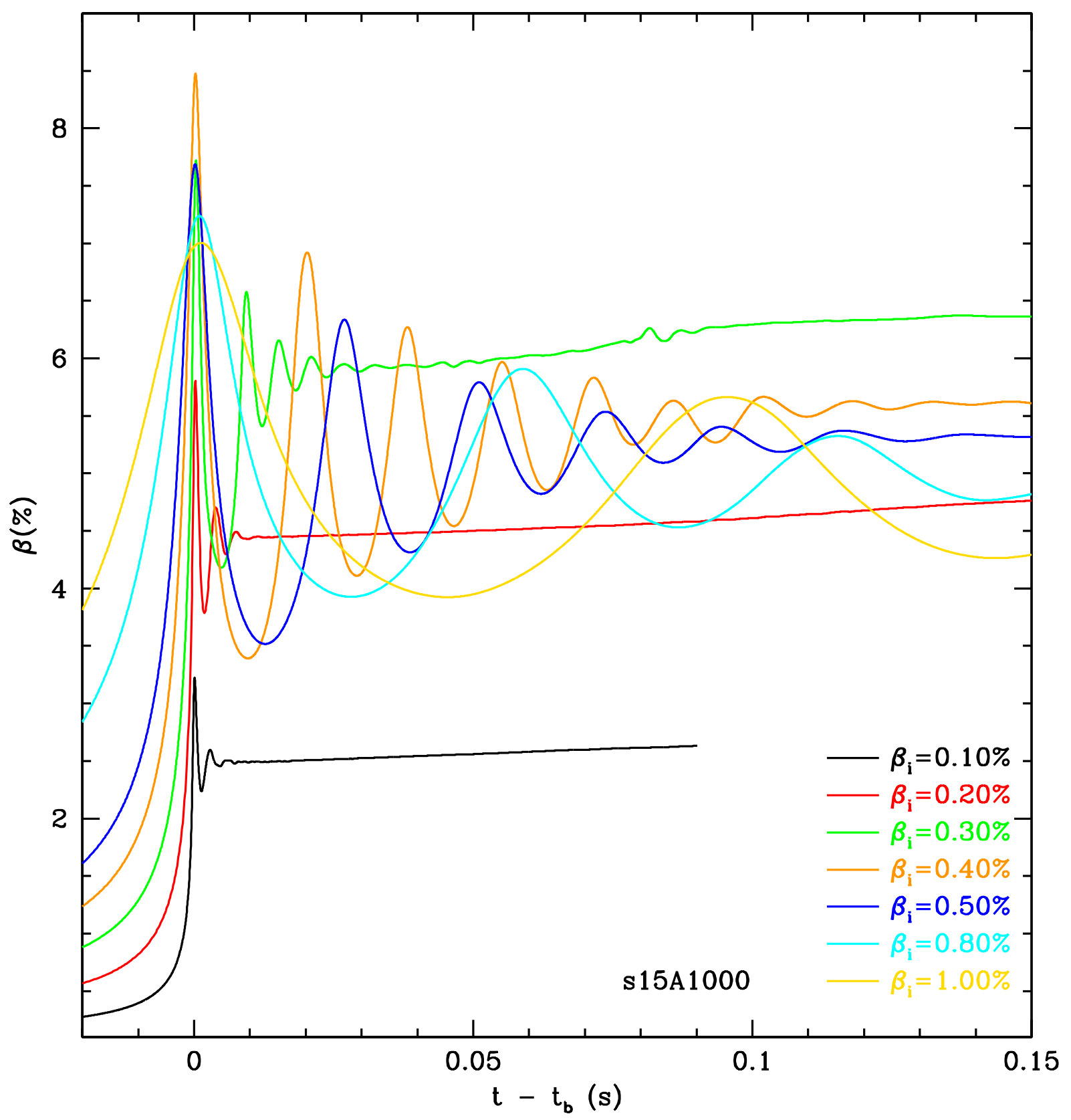

FIG. 7.- Evolution of the ratio of rotational to gravitational energy (rotation parameter $\beta$ ) for the s15A1000 model series. The zero of the time axis is set to the time of bounce for each model. The $\beta_{i}=0.40 \%$ model, which is the first to bounce by centrifugal forces, reaches the maximum $\beta$ at bounce for all models. The $\beta_{i}=0.30 \%$ model has a smaller $\beta$ at bounce but shows, due to the greater compactness of its remnant, the largest final $\beta$. The models with $\beta_{i}>0.40 \%$ collapse so slowly that centrifugal forces are able to halt collapse before greater compression can lead to very large $\beta$ s. None of our models exceeds a $\beta$ of $10 \%$. 

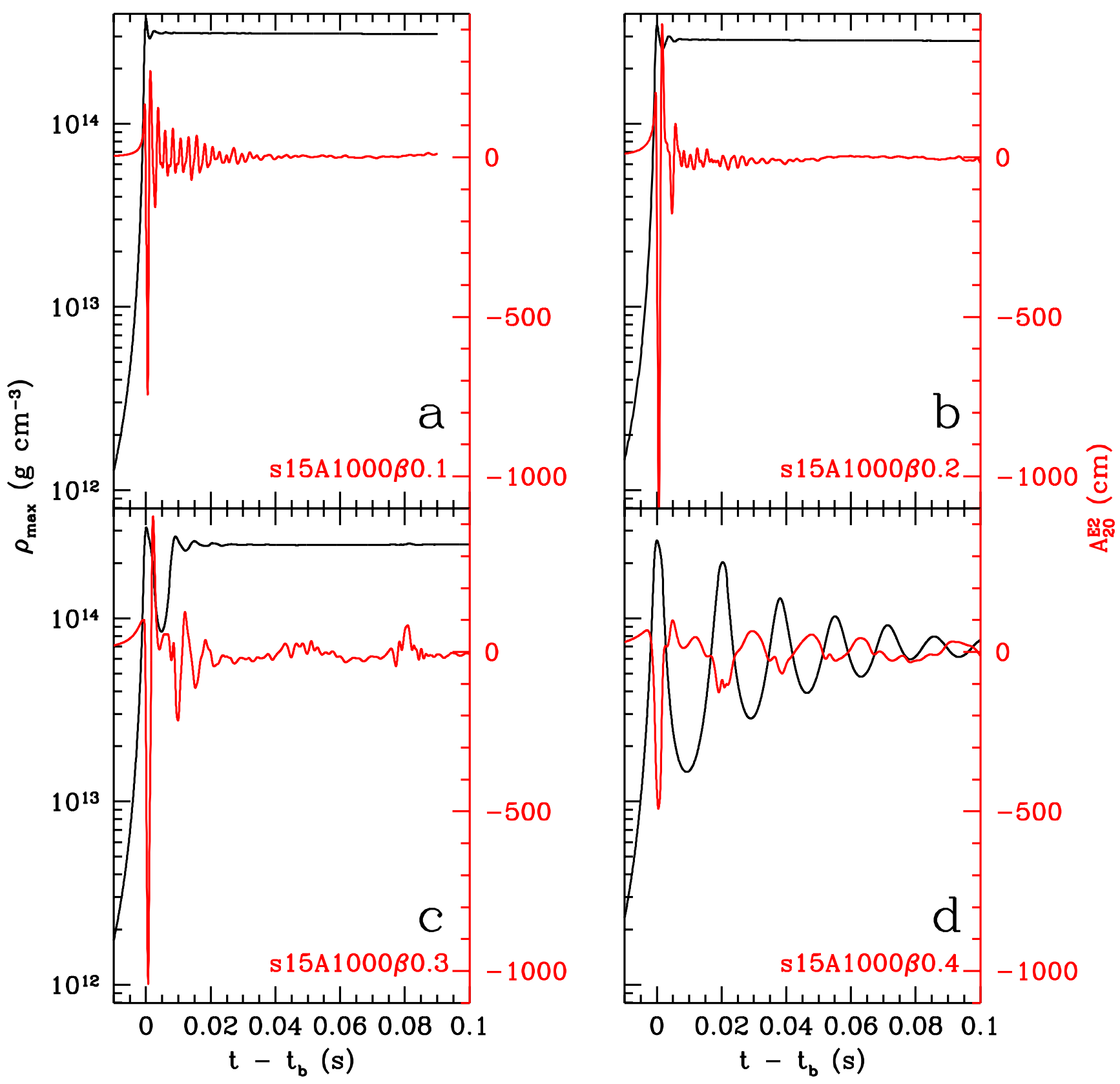

FIG. 8.- Maximum density ( $\rho_{\max }$, black) and gravitational wave amplitude $\left(A_{20}^{E 2}\right.$, red) as functions of time for the Woosley and Weaver (1995) s15 model and $\mathrm{A}=1000 \mathrm{~km}$. For each model, the zero of the time axis is set to the time of core bounce $\left(\mathrm{t}_{b}\right)$. a (upper left): $\beta_{i}=0.10 \%$, bounce at supranuclear density with negligible influence of centrifugal forces (type I). Note the subsequent ring-down waveform, which is associated with both radial- and non-radial modes of the compact remnant. b (upper right): $\beta_{i}=0.20 \%$, similar to the previous model, but with greater asphericity leading to larger amplitudes. c (lower left): Transitional model with $\beta_{i}=0.30 \%$, which is beyond the $\beta_{i}$ for the largest gravitational wave amplitudes. The typical oscillation period of the waveform is significantly larger than those of the previous models and the hydrodynamic data exhibit at least one additional coherent large scale expansion-collapse-bounce cycle caused by the growing influence of centrifugal forces. $\mathbf{d}$ (lower right): $\beta_{i}=0.40 \%$. The hydrodynamic evolution and the associated waveform of this model are already largely influenced by centrifugal forces. The core exhibits subsequent multiple coherent bounces that are quasi-exponentially damped (type II). 

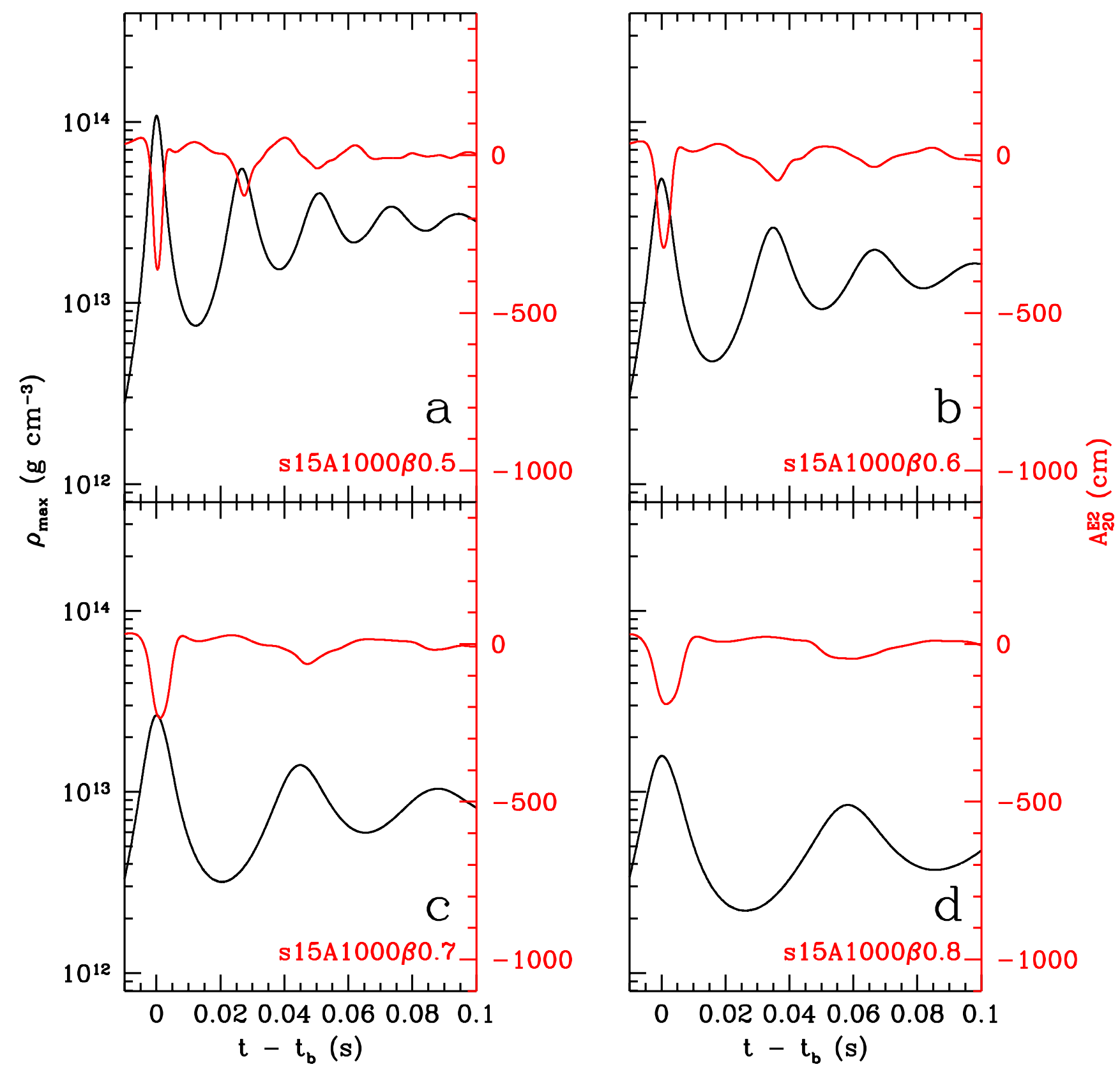

FIG. 9.- Same as Figure 8, but for higher values of $\beta_{i} . \rho_{\max }(t)$ (black) is the maximum density and $A_{20}^{E 2}(t)$ (red) is the gravitational wave amplitude for s15A1000. a (upper left): This model with $\beta_{i}=0.50 \%$ undergoes a bounce at subnuclear density when centrifugal forces overcome gravitational attraction. Note the significantly larger dynamical timescales compared with models with smaller $\beta_{i}$. Panels b, $\mathbf{c}$ and d: As $\beta_{i}$ increases from $0.60 \%$ to $0.80 \%$, bounce occurs more slowly and at progressingly lower densities. The waveforms are dominated by the subsequent expansion-collapse-bounce cycles of the quickly spinning deformed stellar core (type II). 


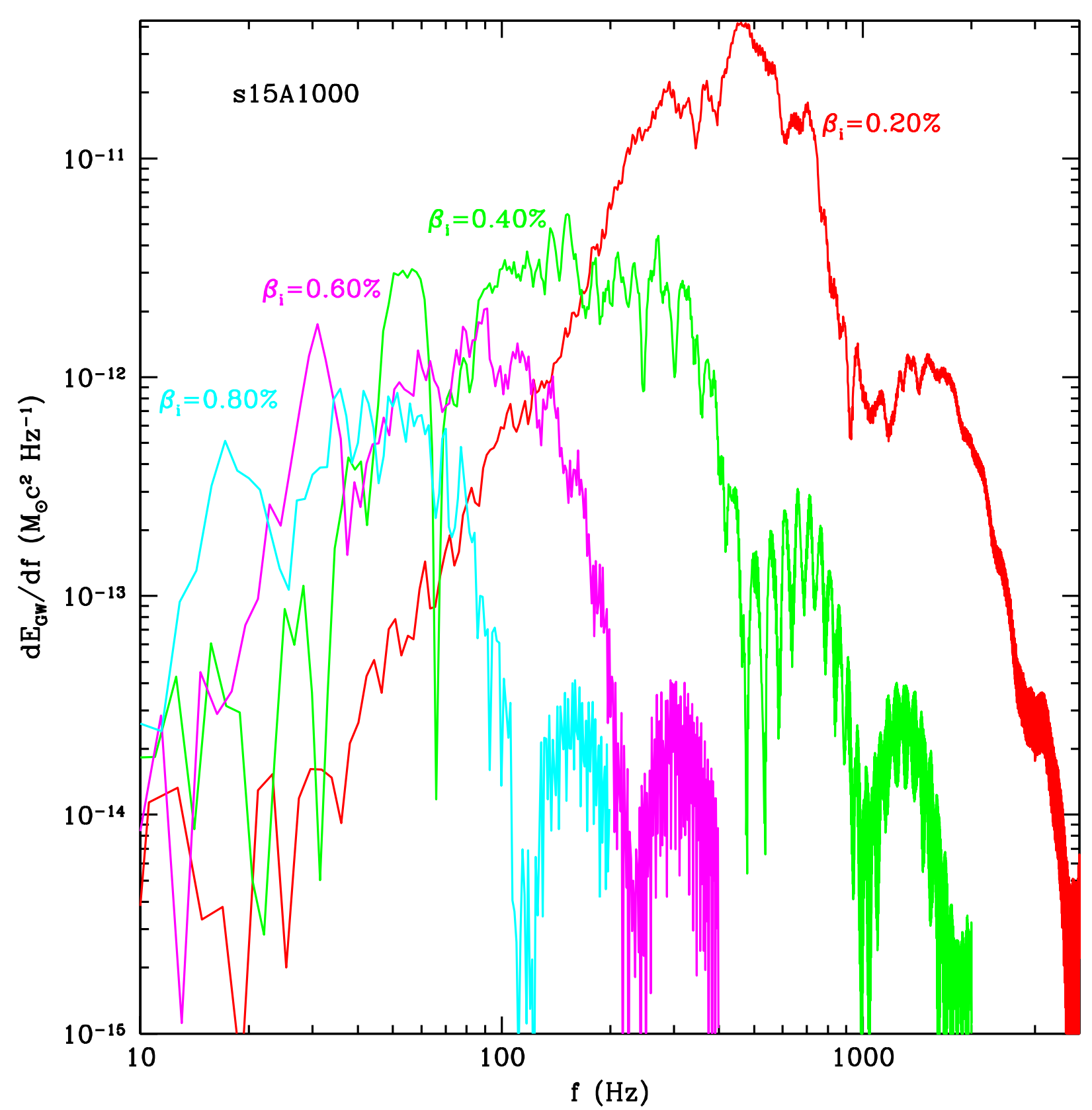

FIG. 10.- Energy spectra of the gravitational radiation emitted from representative models of the s15A1000 model series. Note the shift of the spectra to lower frequencies with increasing $\beta_{i}$ and the logarithmic scale of the ordinate. The spectra have been cut off at a frequency beyond which generic high frequency noise at constant magnitude sets in. The first pronounced peak in the spectra of the more strongly rotating models can be identified with the frequency of the expansioncollapse-bounce cycles exhibited by these models (type II). The spectra of the slower rotators peak at the dominant frequencies of the post-bounce ringing of the compact remnant. The higher harmonics are clearly seen in these models. 

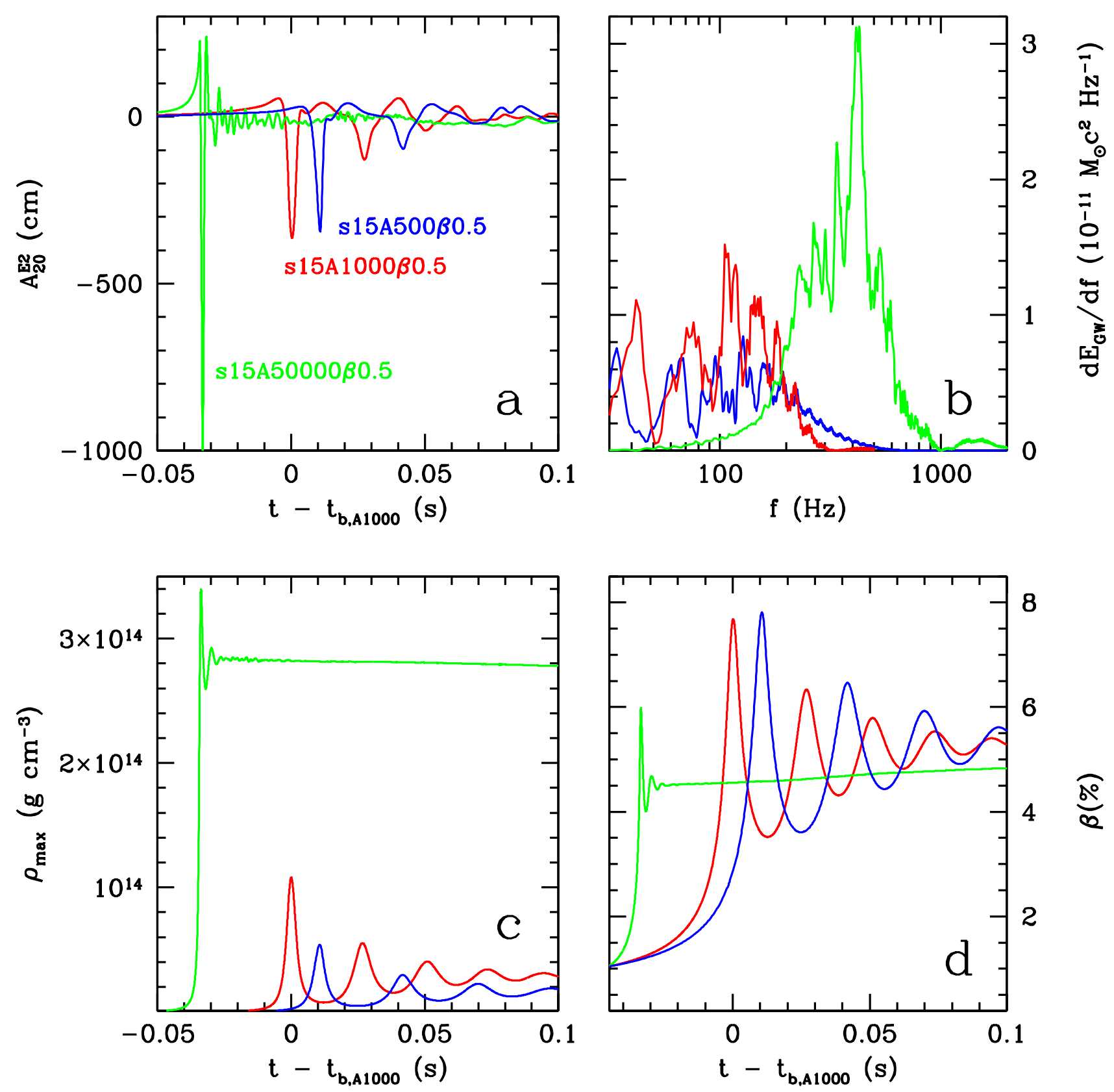

FIG. 11.-Comparison between different initial distributions of angular momentum at fixed $\beta=0.50 \%$ for the s15 model from Woosley and Weaver (1995). Models A50000, A1000, and A500 are nearly rigidly rotating interior to 50000, 1000, and 500 kilometers, respectively. a (upper left): Time evolution of the gravitational wave amplitude for the models s15A50000 $\beta 0.5$, s15A1000 $\beta 0.5$, and s15A500 $\beta 0.5$. The time axis is relative to the time of bounce of the s15A $1000 \beta 0.5$ model. For a given model the transition from a bounce dominated by nuclear repulsive forces to one in which centrifugal forces play a significant role occurs at progressively lower $\beta_{i}$ with decreasing A. b (upper right): Energy spectra of the three models. Note the distinct peak of the rigidly rotating s15A50000 $\beta 0.5$ model (green) at about $400 \mathrm{~Hz}$ that is directly associated with the post-bounce ringing of the compact remnant, also seen in the waveform. The spectra of the models with smaller A exhibit a local maximum at low frequencies that also directly correspond to the frequencies of their post-bounce expansion-collapse-bounce cycles. $\underline{\mathbf{c}(l o w e r ~ l e f t):}$ Evolution of the maximum density. Model s15A50000 $\beta 0.5$ still bounces at supranuclear densities, while the two models with smaller A have already made the transition to type II behavior. d (lower right): Evolution of the rotation parameter $\beta$ with the time given relative to the time of bounce of model s15A1000 $\beta 0.5$. The models with $\mathrm{A}=1000$ and $\mathrm{A}=500$ have more angular momentum in their central regions and, hence, are more strongly influenced by centrifugal forces. For a given $\beta_{i}$, they also achieve larger final $\beta$ s and $\beta$ s at bounce. 


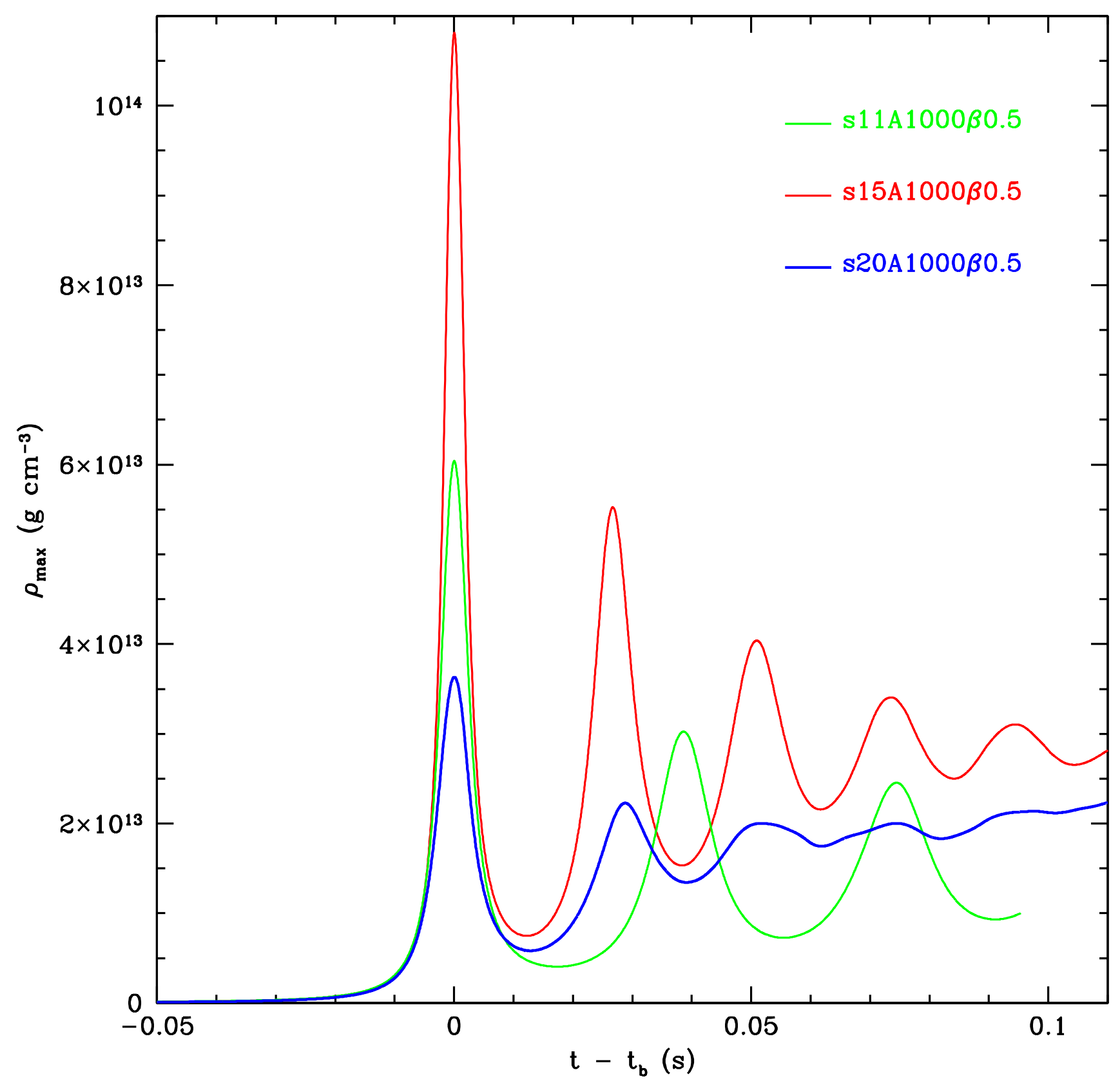

FIG. 12. - Inter-model comparison between s11A1000, s15A1000 and s20A1000 for $\beta_{i}=0.50 \%$ of the evolution of the peak density. The time is given relative to the time of bounce for each individual model. Model s15 reaches higher maximum densities and has the shortest post-bounce oscillation periods. Model s11, which has a similar initial density and angular momentum distribution, reaches lower densities and has longer post-bounce oscillation periods. The s20A1000 model, however, with significantly different initial density, angular momentum, and compositional profiles, bounces for the same A and $\beta_{i}$ at even lower densities. It also has shorter oscillation periods than the s11 model and is more quickly damped by the proximity of its stalled shock. 

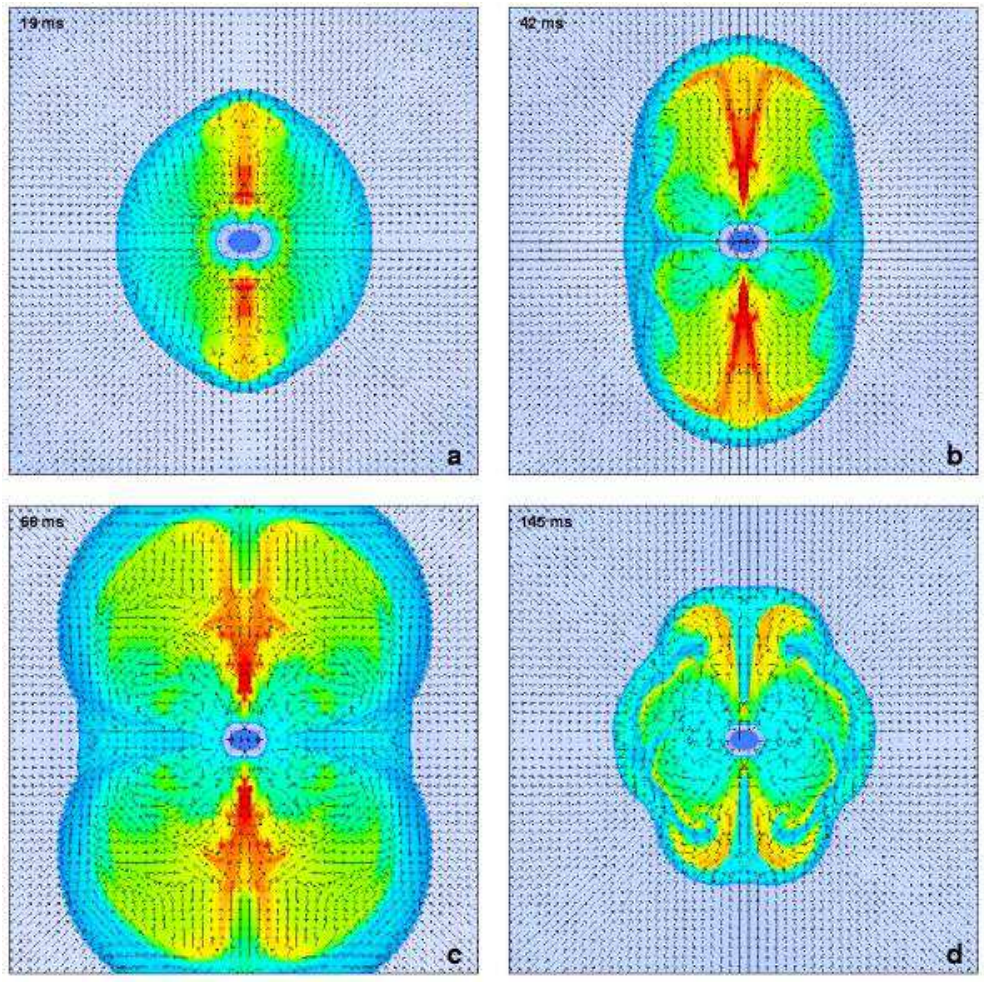

FIG. 13. - 2-D plots of the specific entropy for model s20A1000 $\beta 0.3$. Red denotes high entropy $\left(\sim 14 \mathrm{k}_{B}\right)$ and dark blue denotes low entropy $\left(\sim 0.9 \mathrm{k}_{B}\right.$, seen in the compact remnant). Shown are the inner $1200 \times 1200 \mathrm{~km}^{2}$ of the hydrodynamic grid. As in Fig. 5, velocity vectors are superposed and the times after bounce are given in the top left of each panel. Since the core is oblate, core bounce happens first and at smaller radii along the poles. After bounce, the shock is able to propagate much faster along the rotation axis than in the equatorial region. High entropy, jet-like structures form along the rotation axis. This is seen in panel (a), which shows the core $19 \mathrm{~ms}$ after bounce. The bounce shock has already reached about $400 \mathrm{~km}$ at the poles and $300 \mathrm{~km}$ at the equator. Forty-two ms after bounce (panel b) the axis ratio has increased even more and violent vortical motion has set in. At about $68 \mathrm{~ms}$ after bounce (panel c), the bounce shock has stalled and most velocities point inward. The shock recedes. Panel (d) shows the shocked region at the end of the evolution. The shock has receded even further. Interior to the shock, vortical motion has lead to the breaking of equatorial symmetry. (This figure is available in high-quality format from http://www.ita.uni-heidelberg.de//cott/gwpaper .) 


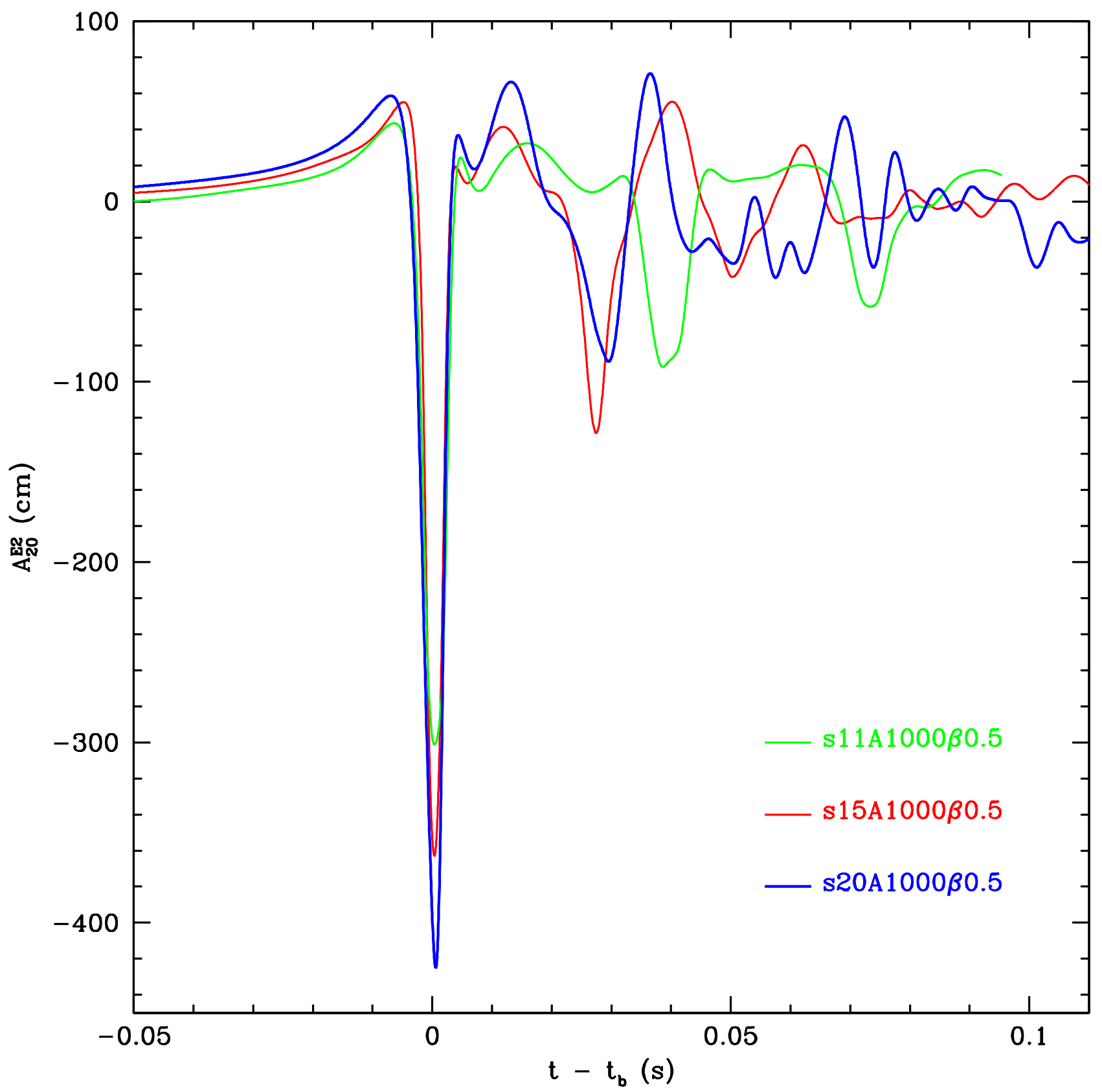

FIG. 14. - Comparison of the evolution of the gravitational wave signal $A_{20}^{E 2}(t)$ of models s $11, \mathrm{~s} 15$ and $\mathrm{s} 20$ for $\mathrm{A}=1000 \mathrm{~km}$ and $\beta_{i}=0.50 \%$. The waveforms reflect the density evolution seen in Fig. 12. For the s20 signal, additional high-frequency contributions appear to be correlated with the vortical motions and aspherical infall seen behind its stalled shock. 


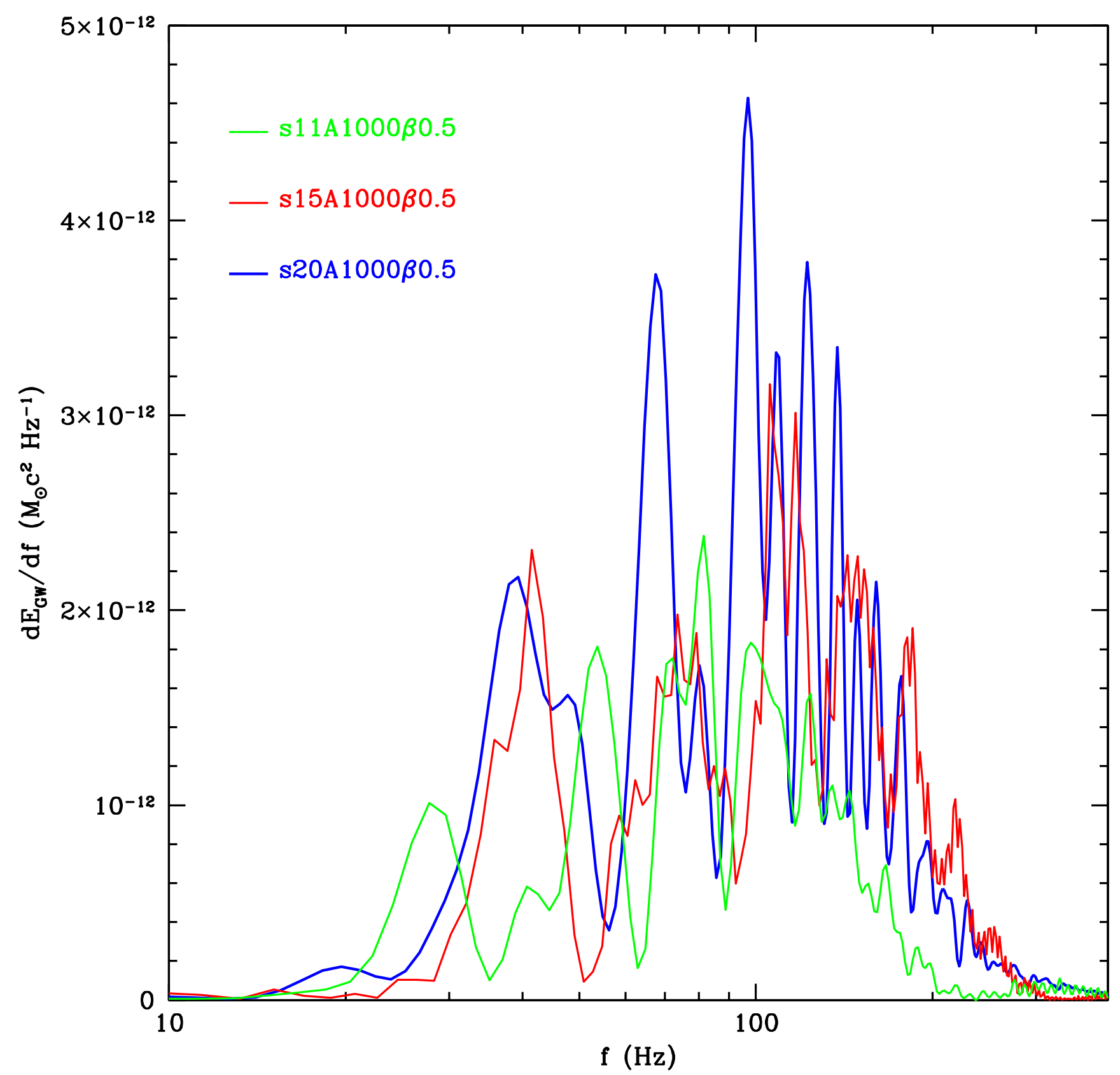

FIG. 15.- Comparison of the gravitational wave energy spectra for s11, s15 and s20 models. Note the linear scale of the ordinate. 

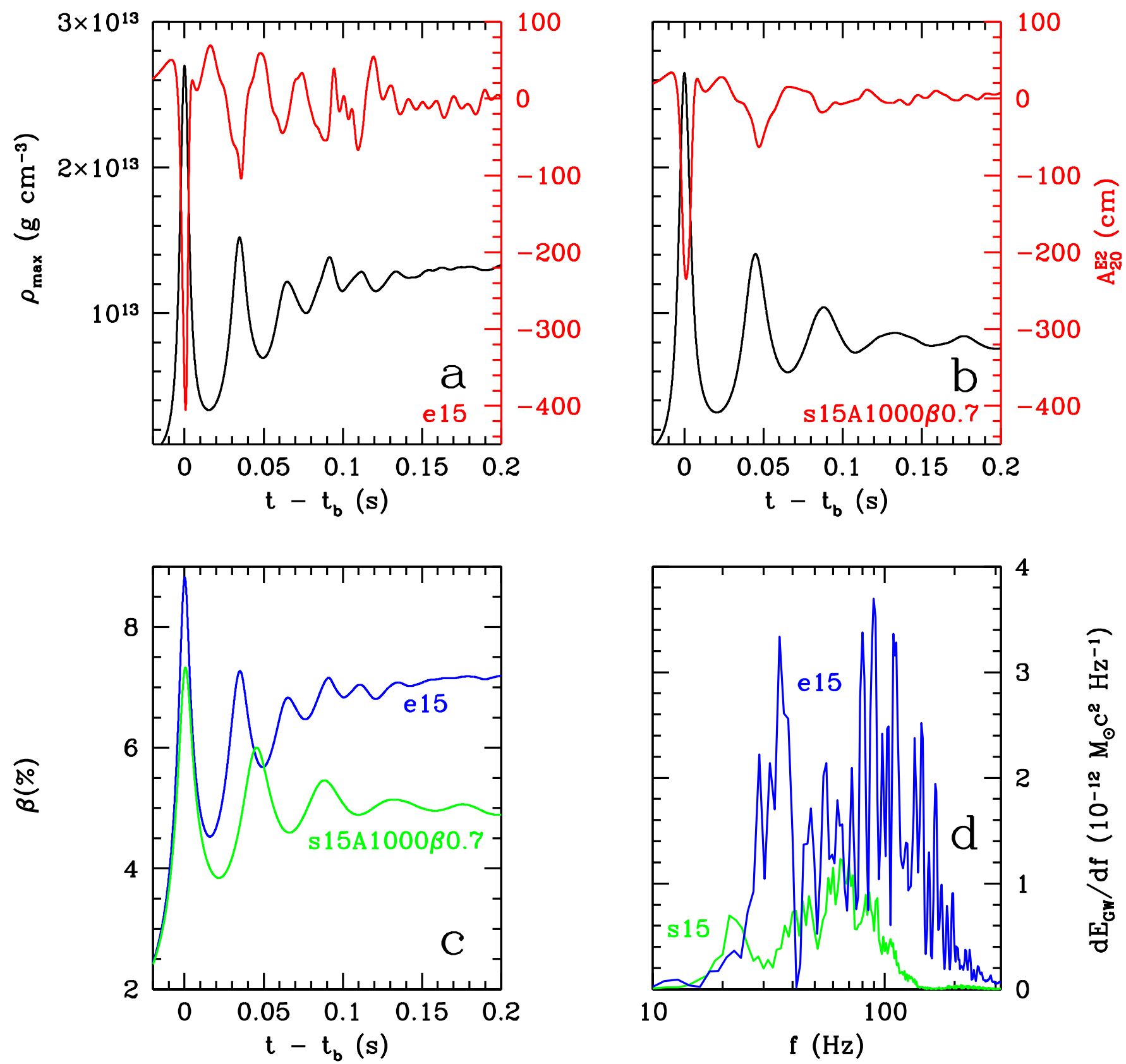

FIG. 16.- Comparison of the e15 model from Heger, Langer, and Woosley (2000) with the s15A1000 $\beta 0.7$ model from Woosley and Weaver (1995). Both models have similar initial $\beta_{i}(0.645 \%$ for the e 15 model versus $0.70 \%$ for the s15A $1000 \beta 0.7$ model $)$ and angular velocity $(\Omega)$ profiles but differ significantly in their total angular momenta. This is due to differences in their initial density profiles. the gravitational wave amplitude, respectively, for models e 15 and s15A1000 $\beta 0.7$. The waveform of e 15 exhibts additional high-frequency components about 100 $\mathrm{ms}$ after bounce that we associated with the sudden damping of the post-bounce expansion-collapse-bounce cycles by infalling matter. This is also reflected in the evolution of the maximum density. c (lower left): Evolution of the rotation parameter $\beta$ for the two models. The larger angular momentum of model e15 and its specific distribution translate into a larger final $\beta$. d (lower right): Energy spectra for the two models under consideration. The e15 model radiates significantly more energy and has distinctly more fine spectral structure. 

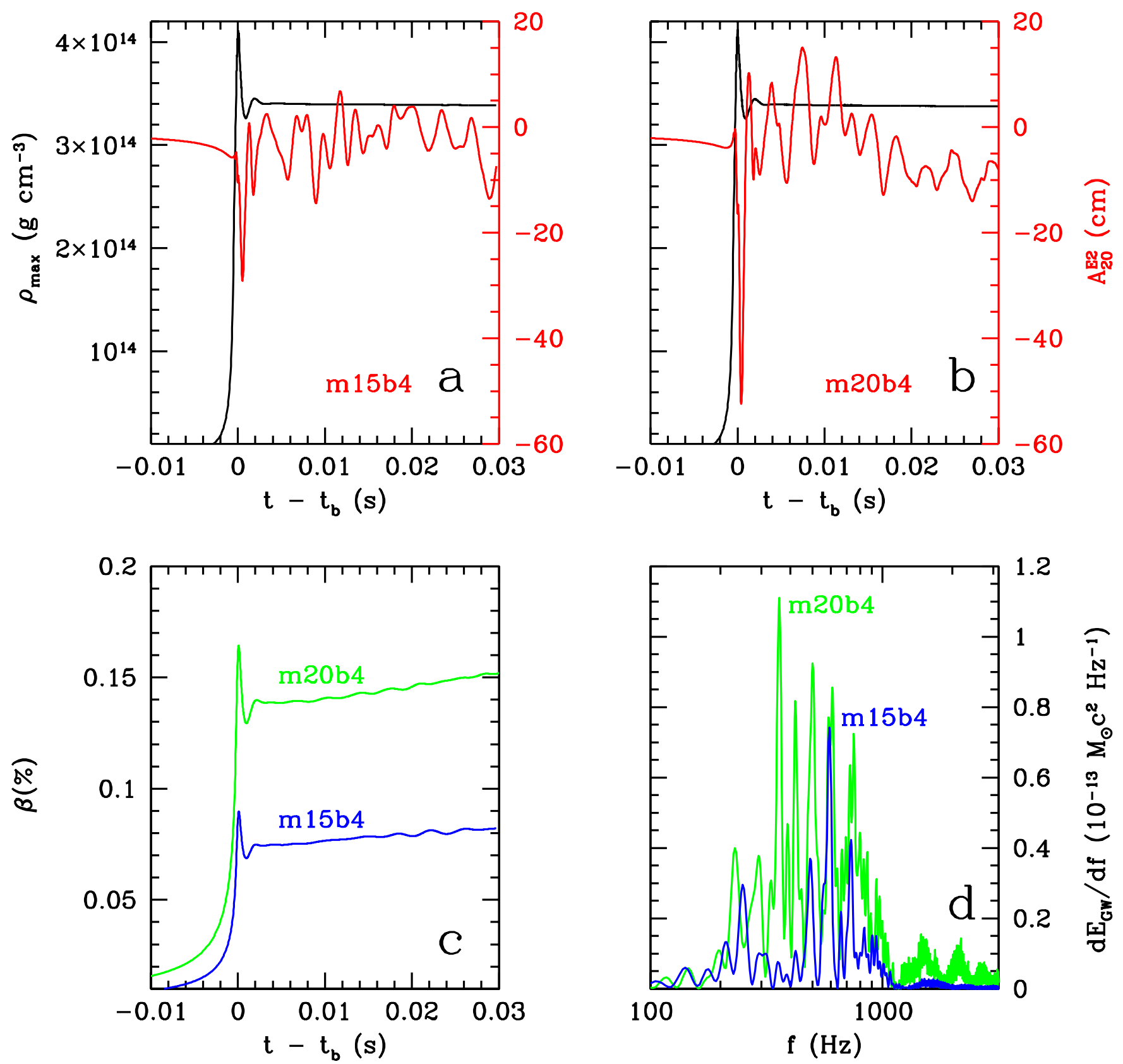

FIG. 17.- The 15 and $20 \mathrm{M}_{\odot}$ models from Heger et al. (2003). a (upper left): Evolution of the maximum density (black) and the gravitational wave amplitude (red) of the m15b4 model. b (upper right): The same for the $\mathrm{m} 20 \mathrm{~b} 4$ model. Both models rotate very slowly and rigidly and show only small deviations from spherical symmetry. Hence, the gravitational wave amplitudes are small. c (lower left): This figure depicts the evolution of the rotation parameter $\beta$ for these models. Both models bounce due to the stiffening of the equation of state at nuclear density, with little contribution due to centrifugal forces. The model with the greater $\beta_{i}, \mathrm{~m} 20 \mathrm{~b} 4$, reaches a larger final $\beta$. d (lower right): Energy spectra. The spectrum of the $\mathrm{m} 20 \mathrm{~b} 4$ model peaks at lower frequencies and contains significantly more energy (4 times more; see Table 6) than the specrum of the m15b4 model. This shows the sensitivity of the gravitational wave signature to small differences in the initial rotation profile and stellar structure. 


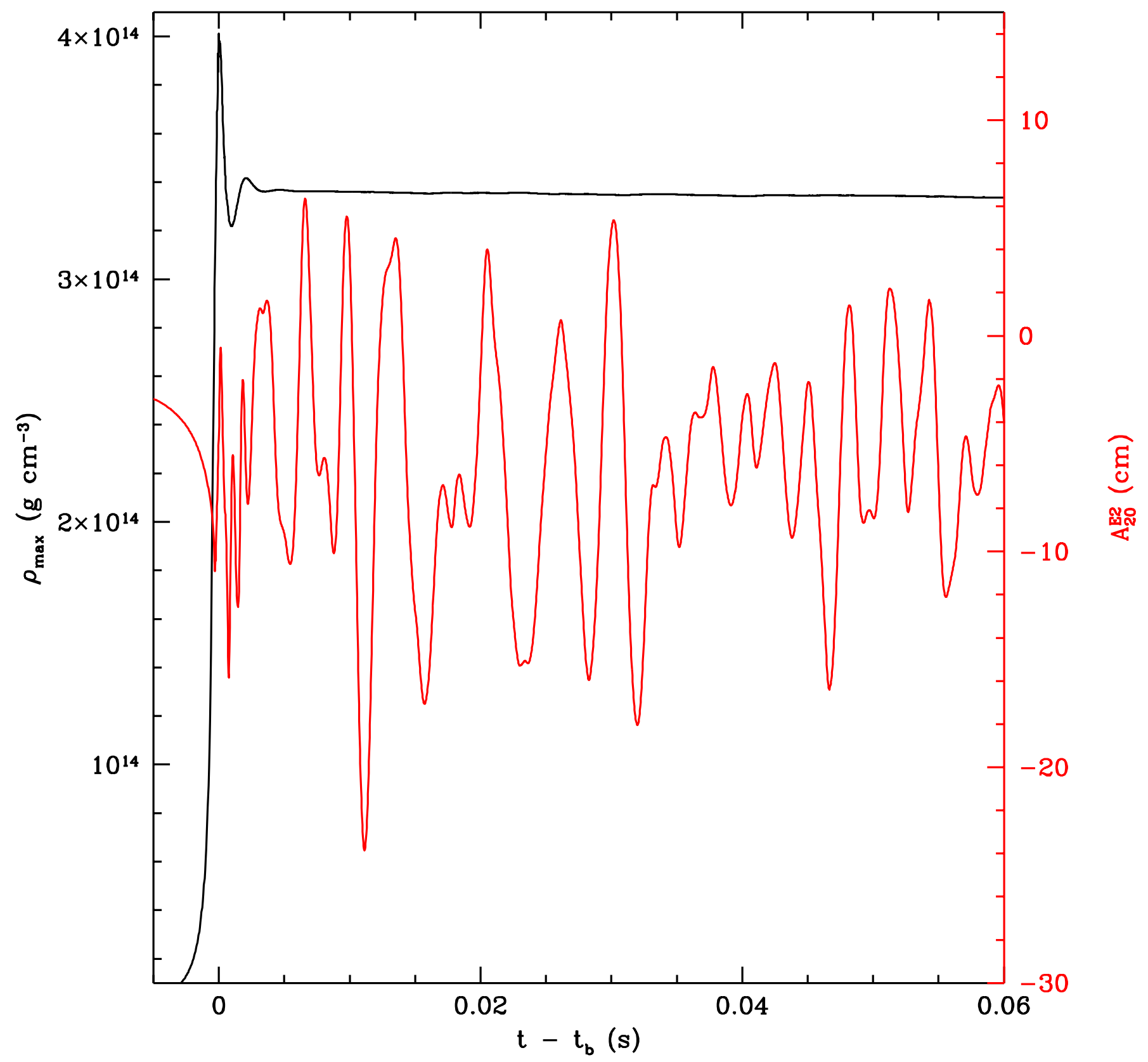

FIG. 18. - Evolution of the maximum density (black) and the gravitational wave amplitude (red) of a s15 model evolved without rotation. The time is given relative to the time of core bounce. Small scale perturbations, introduced by the finite-difference approximation and post-bounce convective instability, lead to continuous gravitational wave emission with amplitudes that are one to two orders of magnitude smaller then those observed from the collapse of a rotating model. 


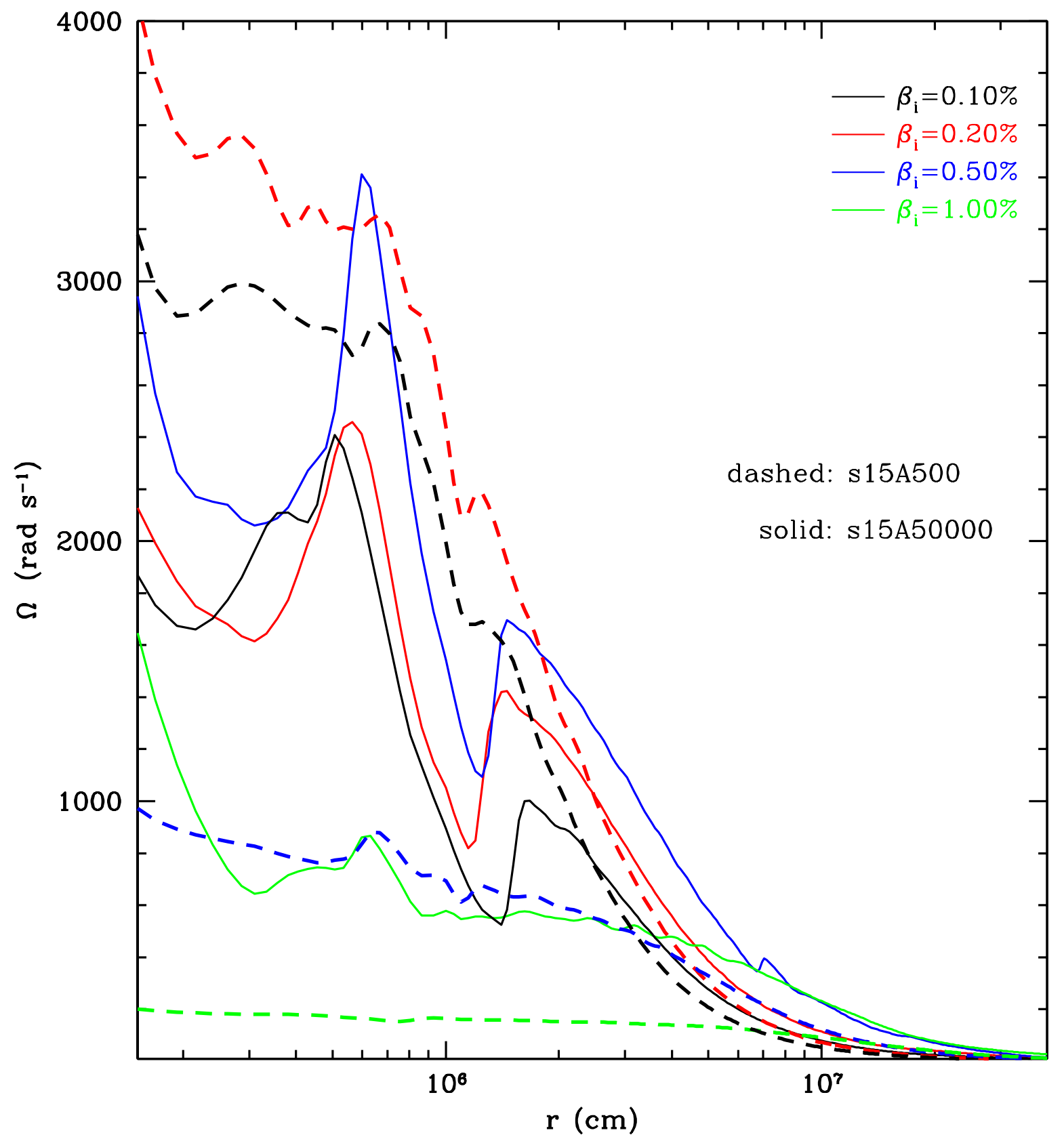

FIG. 19. - Angular velocity profiles in the equatorial region at the end of the evolution of s15 models with $\mathrm{A}=500 \mathrm{~km}$ (dashed) and $\mathrm{A}=50000 \mathrm{~km}$ (solid) and for $\beta_{i} \mathrm{~s}$ of $0.10,0.20,0.50$, and $1.00 \%$. Interestingly, the initially rigidly rotating models $(\mathrm{A}=50000 \mathrm{~km})$ exhibit a more differentially rotating central region than the initially more differentially rotating models do. The local peak in the angular velocity at $\sim 6-8 \mathrm{~km}$ and the strong $\Omega$-gradients associated with it have been considered possible drivers of the magneto-rotational instability (MRI) (Akiyama et al. 2003). 


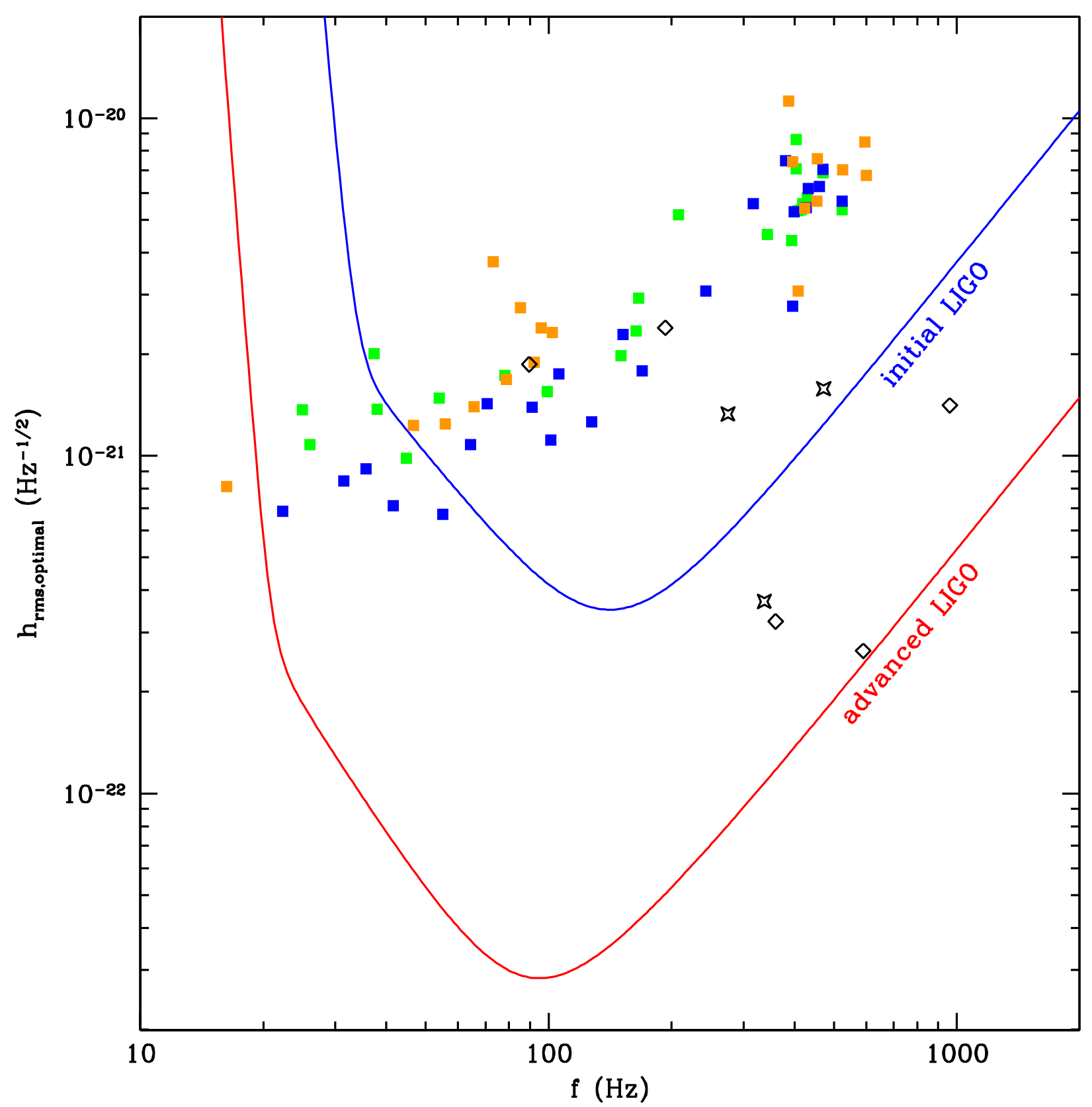

FIG. 20.- LIGO sensitivity plot. Plotted are the optimal root-mean-square noise strain amplitudes $h_{r m s}=\sqrt{f S(f)}$ of the initial and advanced LIGO interferometer designs. Optimal means that the gravitational waves are incident at an optimal angle and optimal polarization for detection and that there are coincident measurements of gravitational waves by multiple detectors. For gravitational waves from burst sources incident at random times from a random direction and a signal-to-noise ratio (SNR) of 5, the rms noise level $h_{r m s}$ is approximately a factor of 11 above the one plotted here (Abramovici et al. 1992; Flanagan and Hughes 1998). We have plotted solid squares at the maxima of the characteristic gravitational wave strain spectrum $\left(h_{\text {char }}(f)\right.$; §8) of our s11 (green), s15 (blue), and s20 (orange) models from Woosley and Weaver (1995) that were artificially put into rotation. Our nonrotating models are marked with stars; diamonds stand for models from Heger, Langer, and Woosley (2000) and Heger et al. (2003). The distance to Earth was set to $10 \mathrm{kpc}$ for all models. Most of our models lie above the optimal design sensitivity limit of LIGO I. Hence, the prospects for detection are good. Those models that are not detectable by the 1st-generation LIGO are those that rotate most slowly (the Heger et al. 2003 models) and those which are the fastest rotators. 


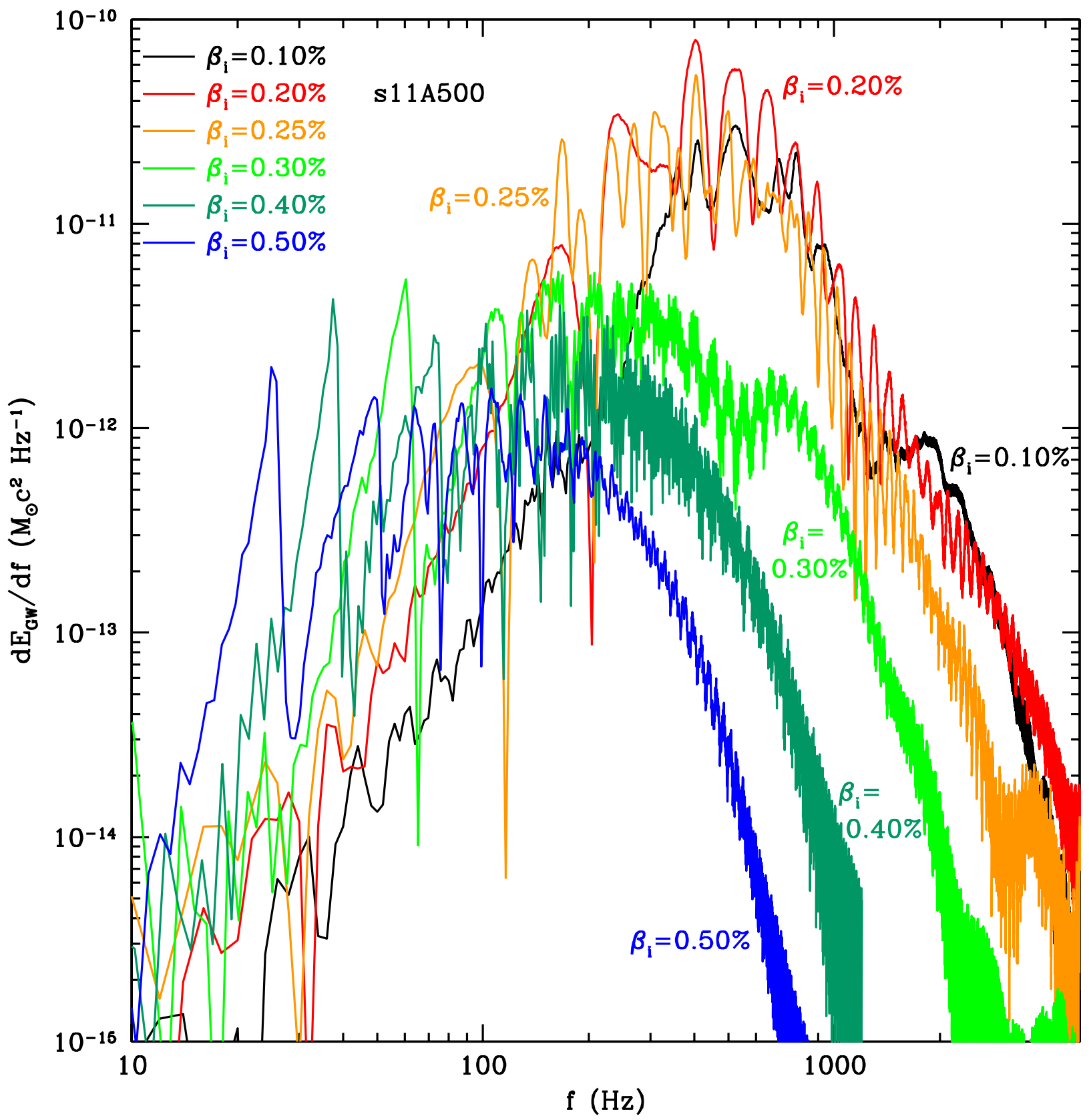

FIG. 21.- Same as Fig. 10, but for the s11A500 sequence and for $\beta_{i}$ s from $0.1 \%$ to $0.5 \%$. 


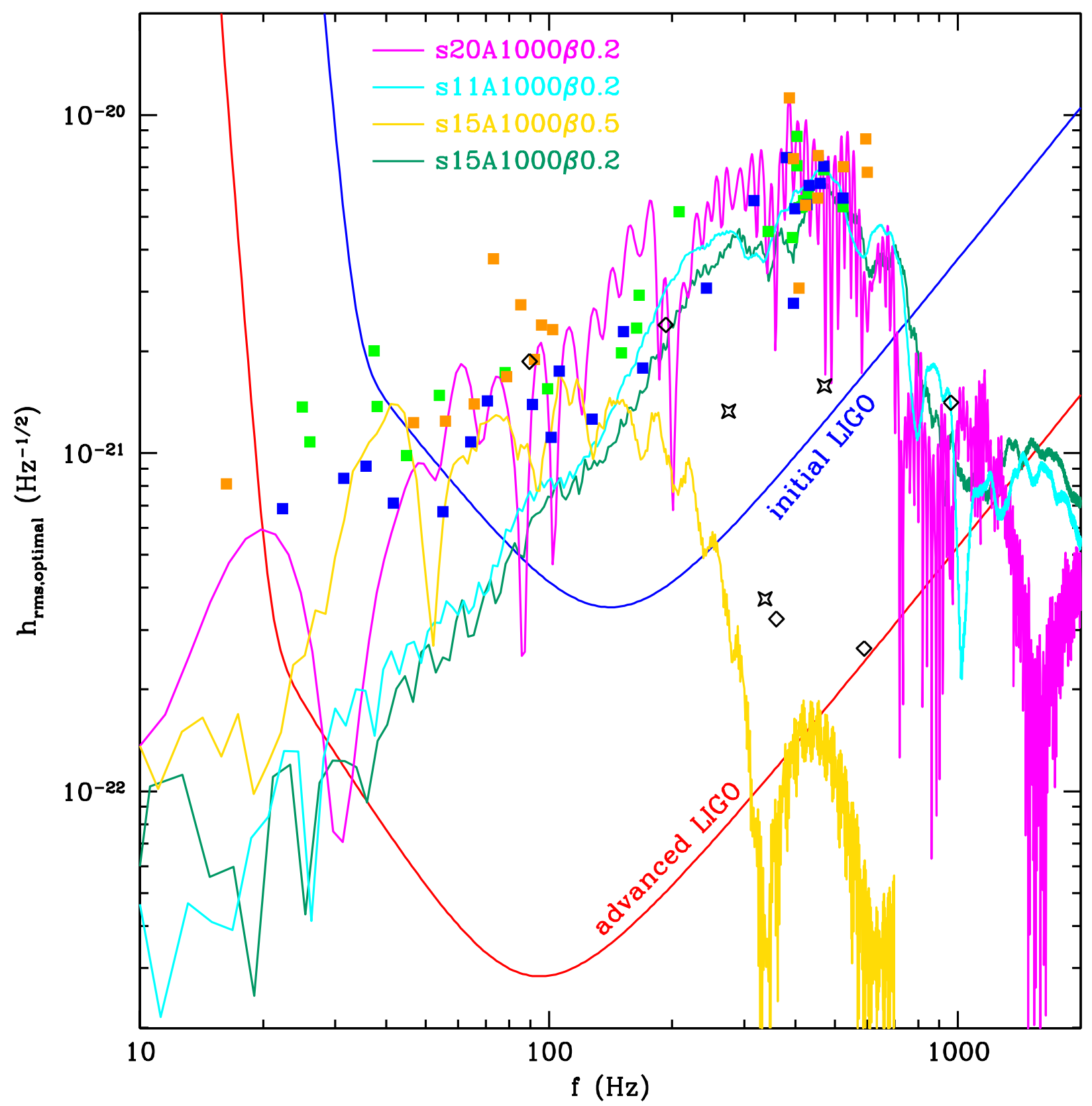

FIG. 22.- Same as Fig. 20, but with various full $h_{\text {char }}$ spectra (using eq. 25) superposed. This plot makes clear the large width of actual spectra and the deviation from even quasi-periodic behavior of rotating collapse wave signatures. 